University of Louisville

ThinkIR: The University of Louisville's Institutional Repository

Electronic Theses and Dissertations

$12-2016$

\title{
Spreading speeds along shifting resource gradients in reaction- diffusion models and lattice differential equations.
}

Jin Shang

University of Louisville

Follow this and additional works at: https://ir.library.louisville.edu/etd

Part of the Biology Commons, Non-linear Dynamics Commons, Ordinary Differential Equations and Applied Dynamics Commons, and the Partial Differential Equations Commons

\section{Recommended Citation}

Shang, Jin, "Spreading speeds along shifting resource gradients in reaction-diffusion models and lattice differential equations." (2016). Electronic Theses and Dissertations. Paper 2593.

https://doi.org/10.18297/etd/2593

This Doctoral Dissertation is brought to you for free and open access by ThinkIR: The University of Louisville's Institutional Repository. It has been accepted for inclusion in Electronic Theses and Dissertations by an authorized administrator of ThinkIR: The University of Louisville's Institutional Repository. This title appears here courtesy of the author, who has retained all other copyrights. For more information, please contact thinkir@louisville.edu. 


\title{
SPREADING SPEEDS ALONG SHIFTING RESOURCE GRADIENTS IN REACTION-DIFFUSION MODELS AND LATTICE DIFFERENTIAL EQUATIONS
}

\author{
By \\ Jin Shang \\ M.A., University of Louisville, 2012 \\ A Dissertation \\ Submitted to the Faculty of the \\ College of Arts and Sciences of the University of Louisville \\ in Partial Fulfillment of the Requirements \\ for the Degree of
}

Doctor of Philosophy in Applied and Industrial Mathematics

Department of Mathematics

University of Louisville

Louisville, Kentucky

December 2016 
Copyright 2016 by Jin Shang

All rights reserved 



\title{
SPREADING SPEEDS ALONG SHIFTING RESOURCE GRADIENTS IN REACTION-DIFFUSION MODELS AND LATTICE DIFFERENTIAL EQUATIONS
}

\author{
By \\ Jin Shang \\ M.A., University of Louisville, 2012
}

A Dissertation Approved On

Date: July 20, 2016

by the following Dissertation Committee:

Dissertation Director: Bingtuan Li

\begin{tabular}{c}
\hline Mary Elizabeth Bradley \\
\hline Ryan Gill \\
\hline Changbing Hu \\
\hline Susanna Remold
\end{tabular}




\section{ACKNOWLEDGEMENTS}

I would like to thank my dissertation advisor, Dr. Bingtuan Li, for his guidance, patience, immeasurable effort in advising me in this doctoral research and valuable comments for revision of this dissertation. I would also like to express my gratitude to Dr. Changbing Hu, Dr. Ryan Gill, Dr. Mary Elizabeth Bradley and Dr. Susanna Remold for their continuous support as committee members and their thought-provoking comments.

I would like to thank my manager Beth Rogers in Data Science team and also my coworkers Cameron White and Cody Kirk for their understanding, support and help for completing my doctoral degree and my dissertation revision.

I wish to thank my family for all of the sacrifices that they've made on my behalf. In particular, I thank my mother in law, Peggy Otto, who is an English professor at WKU, for providing me expert suggestions for dissertation writing. I would also like to thank to my husband, Garrett Otto, for his patience, love, support and always being so supportive on not only my study but also my life and dream. To my beloved son Carter Otto, I would like to express my thanks for being such a good boy always cheering me up. 


\begin{abstract}
SPREADING SPEEDS ALONG SHIFTING RESOURCE GRADIENTS IN REACTION-DIFFUSION MODELS AND LATTICE DIFFERENTIAL EQUATIONS
\end{abstract}

Jin Shang

July 20, 2016

A reaction-diffusion model and a lattice differential equation are introduced to describe the persistence and spread of a species along a shifting habitat gradient. The species is assumed to grow everywhere in space and its growth rate is assumed to be monotone and positive along the habitat region. We show that the persistence and spreading dynamics of a species are dependent on the speed of the shifting edge of the favorable habitat, $c$, as well as $c^{*}(\infty)$ and $c^{*}(-\infty)$, which are formulated in terms of the dispersal kernel and species growth rates in both directions. When the favorable habitat edge shifts towards the right, $c>0$, we demonstrate that the rightward spreading speed is $c^{*}(\infty)$ when $c$ is relatively small and is $c^{*}(-\infty)$ when $c$ is relatively large, and the leftward spreading speed is $c^{*}(-\infty)$. When the favorable habitat edge shifts towards the left, $c<0$, we show that the rightward spreading speed is $c^{*}(\infty)$, and the leftward spreading speed is one of $|c|, c^{*}(-\infty)$ or $c^{*}(\infty)$. We also show the persistence and spreading dynamics of two competing species along shifting habitats in the simplest situations. Their spreading behavior will be affected by the resource distribution and habitat shifting speed. 


\section{TABLE OF CONTENTS}

Page

ACKNOWLEDGEMENTS

ABSTRACT

LIST OF FIGURES vii

\section{CHAPTER}

$\begin{array}{lll}\text { I INTRODUCTION } & 1\end{array}$

II REACTION-DIFFUSION MODEL ANALYSIS 6

1 Reaction-Diffusion Model . . . . . . . . . . . . . 6

2 Reaction-Diffusion Model with Shifting Habitat for One Species 9

3 Upper Solutions . . . . . . . . . . . . . . . . . . . . . . 13

4 Lower Solutions . . . . . . . . . . . . . . . . . . . . 21

$5 \quad$ Spreading Speed . . . . . . . . . . . . . . . . . . . . . 29

6 Simulations for One Species Models . . . . . . . . . . . . . . 44

7 Discussion for Reaction-Diffusion Chapter . . . . . . . . . . 46

III LOTKA-VOLTERRA COMPETITION MODEL ANALYSIS 48

1 Lotka-Volterra Competition Model . . . . . . . . . . . . . 48

2 Lotka-Volterra Competition Model with Shifting Habitat . . . 49

3 Non-persistence . . . . . . . . . . . . . . . . . . . 53

4 Spreading Speed . . . . . . . . . . . . . . 56

5 Discussion for Lotka-Volterra Competition Model Chapter . . . 60

6 Simulations for two species model . . . . . . . . . . . . . . . 62 
IV LATTICE DIFFERENTIAL MODEL ANALYSIS 70

1 Linear Lattice Differential Equations and Modified Bessel Functions 70

2 Well-posedness of Lattice-Differential Model with Shifting Habitat 73

3 Upper Solutions . . . . . . . . . . . . . . . . . 77

4 Lower Solutions . . . . . . . . . . . . . . . . . . . 86

$5 \quad$ Spreading Speed . . . . . . . . . . . . . . . . 94

6 Discussion for Lattice Differential Model Chapter . . . . . . . . 107

V DISCUSSION AND FUTURE DIRECTIONS 108

1 Conclusion . . . . . . . . . . . . . . . . 108

2 Discussion ...................... 109

$3 \quad$ Future Directions . . . . . . . . . . . . . . . . . . . . 112

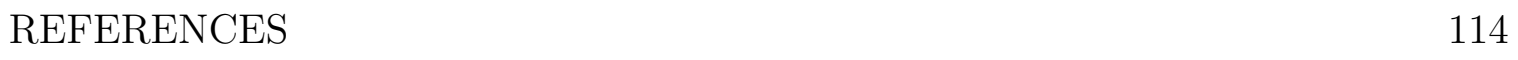

CURRICULUM VITAE 126 


\section{LIST OF FIGURES}

FIGURE

Page

1 Numerical Simulation for Reaction-Diffusion Equations . . . . . . . . 47

2 Numerical Solution for Model (46) when $r_{1}^{+}=4, r_{2}^{-}=1, r_{1}^{-}=r_{2}^{+}=$ $-0.1, d_{1}=0.5, d_{2}=1, a_{1}=1, a_{2}=2$, and $c=3 \ldots \ldots$.

3 Numerical Solution for Model (46) when $r_{1}^{+}=4, r_{2}^{-}=1, r_{1}^{-}=r_{2}^{+}=$ $-0.1, d_{1}=1.5, d_{2}=1, a_{1}=1, a_{2}=2$, and $c=2.2 \ldots \ldots$

4 Numerical Solution for Model (46) when $r_{1}^{+}=4, r_{2}^{-}=2, r_{1}^{-}=r_{2}^{+}=$ $-0.1, d_{1}=1, d_{2}=1, a_{1}=1, a_{2}=2$, and $c=1.7 \ldots \ldots$

5 Numerical Solution for Model (50) when $r_{1}^{+}=1, r_{2}^{+}=2, r_{1}^{-}=r_{2}^{-}=$ $-0.1, d_{1}=1.5, d_{2}=2, a_{1}=0.1, a_{2}=1$, and $c=2 \ldots \ldots$. . . . .

6 Numerical Solution for Model (50) when $r_{1}^{+}=1, r_{2}^{+}=2, r_{1}^{-}=r_{2}^{-}=$ $-0.1, d_{1}=1.5, d_{2}=2, a_{1}=1, a_{2}=1$, and $c=2 \ldots \ldots$

7 Numerical Solution for Model (50) when $r_{1}^{+}=1, r_{2}^{+}=2, r_{1}^{-}=r_{2}^{-}=$ $-0.1, d_{1}=1.5, d_{2}=2, a_{1}=0.1, a_{2}=1$, and $c=-2 \ldots \ldots$

8 Numerical Solution for Model (50) when $r_{1}^{+}=1, r_{2}^{+}=2, r_{1}^{-}=r_{2}^{-}=$ $-0.1, d_{1}=1.5, d_{2}=2, a_{1}=1, a_{2}=1$, and $c=-2 \ldots \ldots$

9 Numerical Solution for Model (50) when $r_{1}^{+}=1, r_{2}^{+}=2, r_{1}^{-}=0.8, r_{2}^{-}=$ $0.6, d_{1}=1.5, d_{2}=2, a_{1}=0.1, a_{2}=1$, and $c=2 \ldots \ldots$

10 Numerical Solution for Model (50) when $r_{1}^{+}=1, r_{2}^{+}=2, r_{1}^{-}=0.8, r_{2}^{-}=$ $0.6, d_{1}=1.5, d_{2}=2, a_{1}=1, a_{2}=1$, and $c=2 \ldots \ldots$ 


\section{CHAPTER I}

\section{INTRODUCTION}

The persistence and spread of a species in an environment have raised fundamental and longstanding questions in ecology. These questions have received more and more attention in recent years since the stability of ecosystems change when confronted with anthropogenic disturbances of the environment. Such anthropogenic disturbances include climate warming, landscape conversion, species invasion, pollution and so on. Anthropogenic disturbances have been blamed for widespread population decline (Parmesan 2006) and the possible extinction of many species (Dover 2014). At the same time, some species, like pest species and alien invasive species (Morrison et al. 2005; Bradley et al. 2010; Paradis et al. 2008), are well adapted to thrive as a result of specific disturbances caused by human activities. It is interesting to see the conditions under which the species survive and spread in the environment and what can cause their population to decline when the environment changes.

In early studies of species' persistence and spread of the population system, it was recognized that the spatial context, especially the combined effect of habitat range and species dispersal, is one of the most important factors. Fisher's equation (Fisher 1937; Kolmogorov et al. 1937) was introduced as the prototypical framework to study spatial population ecology. This framework can be used to describe the propagation of a virile mutant in an infinitely long habitat (Fisher 1937) and can be applied to analyze the population behavior for any mobile species (Al-Khaled 2001). In order to analyze different biological problems, there are various extensions of Fish-

er's equation. For example, Fisher's equation was extended to model a system with a general growth function by Aronson and Weinberger (1975) and was also implemented in multiple dimension systems (Aronson and Weinberger 1978; Weinberger 
et al. 2002), specifically in the system with interacting species (Fagan et al. 1999; Weinberger et al. 2002; Lewis et al. 2002; Li et al. 2005). The modified versions of Fisher's equation have been applied to discrete time systems in order to capture seasonal life-histories and systems incorporating spatial heterogeneity or periodicity (Shigesada et al. 1986; Andow et al. 1990; Weinberger 2002; With 2002; Fagan et al. 2009; Huang and Shen 2009).

Attention has also been paid to analyzing the relationship between biological invasion and climate change (Hobbs 2000; Rahel and Olden 2008). It was recognized that climate change can result in habitat shifts (Polovina et al. 2011; Parr et al. 2012), expansions (Ni 2000; Gonzalez et al. 2010; Lynch et al. 2012), and contractions (Ni 2000; Gonzalez et al. 2010; Parr et al. 2012). A vast body of literature studies changes in the boundaries between forest and non-forest habitat types (e.g. Scheiter et al. 2009; Gonzalez et al. 2010; Scheffer et al. 2012; Tng et al. 2012). Due to rapid warming on the earth in the past few decades, many animals have shifted their ranges northward or to higher altitudes, while for those animals that already live in the Arctic, their habitat shrinks year after year. In other cases, suitable habitat for some species are even expanding (Ni 2000; Gonzalez et al. 2010). This happens frequently for invasive species. For example, the hemlock woolly adelgid (HWA) was accidentally introduced from Japan into the southeastern United States in 1950. Higher temperatures caused by global warming are good for HWAs' growth, so they have been expanding their habitat into half of the eastern portion of the hemlock's range. Since HWA can kill hemlocks and hemlocks are not suitable for the high temperature area, hemlocks lose their habitat in this process (Orwig 2002).

The rate of habitat change is another important factor for impacting species' persistence and spread. Usually, the habitat changes gradually (e.g., Polovina et al. 2011; Parr et al. 2012). For example, the changes caused by continental drift, and mountain-building occur over long time periods. In other cases, the habitat can shift very fast (Scheffer et al. 2012) or even in response to a single climatic event (Smale and Wernberg 2013), while the species might take a million years to adapt to the climate change. A study (Quintero and Wiens 2013) has shown that climate 
change already exceeds the adaptation capability of many species. Many creatures will face extinction. These threats associated with global change largely drive the current interest in spatial ecology.

Considering climate-driven habitat shifts in spatial ecological models, Berestycki et al. (2009) determined the minimum size of a moving habitat for species persistence. They also expanded their results to discuss the persistence of a species in any region with a shifting habitat. Similar work was done by Potapov and Lewis (2004) to consider the effects of a shifting range boundary on a competition model. The mathematical framework created in Berestycki et al. (2009) was extended to an integrodifference system by Zhou and Kot (2011). They concluded that the species will become extinct if the speed of the range shift exceeds a critical rate.

In this dissertation, we introduce three related mathematical frameworks for studying species range expansion in the context of climate change. In Chapter II, we study a reaction-diffusion equation for a single species, which is based on Fisher's equation to study the impact of climatically driven habitat change for the persistence and spread of an invasive species. In Chapter III, we generalize the mathematical framework of Chapter II to describe the interaction between two competing species. In Chapter IV, we provide the third mathematical framework by utilizing a lattice differential equation, which expands the framework in Chapter II to a discrete space.

This framework of Chapter II, was also discussed in Li et al. (2015). In this paper, the habitat of the species can be divided into two regions: a right region which is suitable for population growth (i.e. a positive growth rate) and a left region which is unsuitable for population survival (i.e. a negative growth rate). The boundary between these two regions was assumed to move toward the right so the suitable habitat for species is drifting rightward with the rate $c>0$. Li et al. (2015) demonstrated that if habitat drift rate is higher than the maximum rate of expansion of the species population, $c>c^{*}(\infty)$, then the species will become extinct in the habitat. When habitat drifts more slowly than $c^{*}(\infty)$, then the species will persist and spread along the shifting habitat gradient at speed $c^{*}(\infty)$. However, the effect in the case that the habitat boundary drifts leftward is not considered in this paper. 
The goal of Chapter II in this dissertation is to extend results in Li et al. (2015) to reaction-diffusion models with more general assumptions. We assume that the species grows everywhere along the spatial gradient, but with differing levels of success. The profile of the habitat quality function remains constant but drifts either right or left at a constant speed $c$ so that we can examine both expansion and contraction of the region of a favorable habitat.

In Chapter III, dealing with a two-species competitive reaction-diffusion model, we see these models can accommodate a wider range of persistence and spread behaviors observed in real systems and their dynamics are more complex than the reaction-diffusion equation and the lattice differential equation. In this Chapter, we mainly discuss the simplest competition model, considering the impact of climate change for persistence and spread of species. The habitat for each species is assumed to be divided into two regions separately: the region which is suitable for population growth and the region which is unsuitable for population growth. We assume both their suitable habitats are drifting in opposite directions, so the impact of the competitive interaction for population persistence and spread will become increasingly weak.

The framework of Chapter IV, dealing with lattice differential equations, was discussed in $\mathrm{Hu}$ and $\mathrm{Li}$ (2015). The lattice differential equation is a modified version of the reaction-diffusion equation where space is divided into discrete patches which can be considered spatially homogenous. Scheel (2009) showed that lattice dynamical systems naturally arise on infinite-dimensional invariant manifolds of reaction-diffusion equations with spatially periodic diffusive fluxes. In $\mathrm{Hu}$ and $\mathrm{Li}$ (2015), modified Bessel functions were initially introduced to express the solution of the lattice equation. They found that the spreading speed of species in the lattice space depends on the shifting rate of the habitat boundary edge when the habitat is drifting rightward. The habitat of the species in this paper can be divided into two regions: a right region which is suitable for population growth (i.e. a positive growth rate) and a left region which is unsuitable for population survival (i.e. a negative growth rate). The boundary between these two regions was assumed to move toward the right so 
the suitable habitat for species is drifting rightward with the rate $c>0$. Similar to reaction-diffusion equations, we will examine both expansion and contraction of the region of a suitable habitat, as well as the case that the species grows everywhere along the spatial gradient, but with differing levels of success. In general, the lattice differential equation could behave differently from the corresponding reaction-diffusion equation(Keener 1987; Wu and Zou 1997).

This dissertation is organized as follows. In the next chapter, we study the reaction-diffusion equation in one dimensional space. We demonstrate that the rightward spreading speed and leftward spreading speed of the species are based on the rate of climate change and the intrinsic rate of spread of the species. We examine the impact of both expansion and contraction of the region of a favorable habitat on the persistence and spread of the species. We also show some important simulations of the model. In Chapter III, a specific Lotka-Volterra competition model is provided and the main mathematical results for this model are presented. Their simulations are provided at the end of this chapter. In chapter IV, we focus on the persistence and spread of species along discrete shifting habitat using lattice differential equations. 


\section{CHAPTER II}

\section{REACTION-DIFFUSION MODEL ANALYSIS}

\section{Reaction-Diffusion Model}

Reaction-diffusion equations first arose in the context of chemistry and subsequently were used to describe dynamic processes of a non-chemical nature. The applications of reaction-diffusion systems can be found in biology, medicine (physiology, diseases, etc.), genetics, physics, social science, finance, economics, weather prediction, astrophysics, and so on (Aronson and Weinberger 1975; Grindrod 1996; Murray 2012; Scott 2003).

In biology, reaction-diffusion equations are used to model the intrinsic reaction activities of a species, such as birth, death, interactions with other species, and movement of a species in a bounded or unbounded domain. Reaction-Diffusion equations are created based on the assumptions that dispersal and growth take place continuously in both space and time and that dispersal is conducted by random diffusion (Lewis and Li 2012). These equations have been utilized in a large portion of mathematical literature on spread and persistence of species (Lewis and Li 2012; Shigesada 1997; Murray 2002a; Murray 2002b; Cantrell and Cosner 2003).

Among the most known reaction-diffusion equations, we introduce the classical

Fisher's equation (also known as Fisher-Kolmogorov- Petrovsky-Piskunoff (FKPP) equation) (Fisher 1937; Kolmogorov et al. 1937) as follows:

$$
\frac{\partial u}{\partial t}=d \frac{\partial^{2} u}{\partial x^{2}}+u(r-u) .
$$

Here $u=u(t, x)$ describes the density/concentration of the species of interest at time $t$ and position $x \in R$, and $r$ is the carrying capacity of the environment. This model contains two primary components: a reaction term $(u(r-u))$ and a diffusion term 
$\left(d \frac{\partial^{2} u}{\partial x^{2}}\right)$. The reaction term $f(u)=u(r-u)$ denotes the local reaction kinetics and describes the process of density $u(t, x)$ changes based on the factors except for diffusion, (i.e. birth, death). The population in this model is governed by the logistic law. For small populations the growth is exponential, but as the population approaches the carrying capacity of the environment, growth slows to zero.

In FKPP model (1), $d \frac{\partial^{2} u}{\partial x^{2}}$ is used to describe the diffusion of the population. Diffusion is the tendency of species to spread out in order to occupy an available space and can be considered as the continuum limit of the 1d random walk which begins at a point and takes a step in a randomly chosen direction. The diffusion coefficient, $d$, is determined by Fick's laws, which address how the diffusive flux goes from regions of high concentration to regions of low concentration (Fick 1855; Cantrell and Cosner 2003). It is easy to show that there are two equilibrium states for the FKPP model, one of which $(u(t, x)=r)$ is stable and another $(u(t, x)=0)$, which is unstable.

One important measure to mathematically analyze the spatial spread of a species is to investigate the speed at which the species spreads into the new environment. Aronson and Weinberger $(1975,1977,1978)$ introduced the concept of spreading speed as a mechanism to quantify the spread. For the typical FKPP reaction-diffusion equation, the spreading speed is defined as follows.

Definition II.1 $c^{*}$ is called the spreading speed of the model (1) if the solutions of model (1) have the following properties:

1. If $0 \leq u(x, 0)<r$ and $u(x, 0) \equiv 0$ for all sufficiently large $|x|$, then for any positive $\epsilon$

$$
\lim _{t \rightarrow \infty}\left\{\max _{|x| \geq\left(c^{*}+\epsilon\right) t} u(x, t)\right\}=0 ;
$$

2. If $0 \leq u(x, 0) \leq r$, and if $u(x, 0) \not \equiv 0$, then for any positive $\epsilon$,

$$
\lim _{t \rightarrow \infty}\left\{\sup _{|x| \leq\left(c^{*}-\epsilon\right) t}|r-u(x, t)|\right\}=0 .
$$

In this definition, the first statement shows that the speed of the traveling wave cannot exceed spreading speed $c^{*}$, which means that if we move faster than 
spreading speed, then we will always be in front of the advancing population and see a population density of 0 . The second statement asserts that the asymptotic spreading speed cannot be slower than $c^{*}$. This means that if we move slower than spreading speed then we will always be behind the leading edge of the advancing population and see a population density of $r$. Therefore, this definition shows that $c^{*}$ is the asymptotic rate of spread of the solutions of the reaction-diffusion equation.

For a general reaction-diffusion system

$$
\begin{aligned}
& \frac{\partial u}{\partial t}=d \frac{\partial^{2} u}{\partial x^{2}}+f(u), \\
& u(0, x)=u_{0}(x),
\end{aligned}
$$

Aronson and Weinberger (1978) showed that, if $f(u)$ describes the logistic population growth, then the spreading speed is given by

$$
c^{*}=2 \sqrt{d f^{\prime}(0)}
$$

so that the spreading speed of model $(1)$ is $c^{*}=2 \sqrt{d r}$. This conclusion was conjectured by Fisher (1937) and was proved by Kmologorov et al. (1937).

Fisher (1937) also showed that the scalar model (1) for the spread of a more fit population into the territory of a less fit one will have traveling wave solutions of all speeds $c \geq c^{*}=2 \sqrt{d r}$. The traveling wave solutions of the FKPP model with speed $c$ are solutions in the form of

$$
u(t, x):=w(x-c t)
$$

where $w(-\infty)=r$ and $w(\infty)=0$, which connect the unstable equilibrium state to the stable equilibrium state. From equation (2), we notice that the traveling wave solution describes the propagation of a species as a wave whose shape remains constant over time, but is translated by a fixed length for each iteration of time.

Similar results that the spreading speed is the slowest speed of a family of traveling waves have been expanded to a more general class of models which includes Fisher's quadratic model as a special case (Weinberger 1982; Aronson and Weinberger 1975; Aronson and Weinberger 1978). Traveling wave solutions can help us to have 
a better understanding how a species propagates in space and it is often easier to calculate the slowest wave speed than to find the spreading speed.

\section{Reaction-Diffusion Model with Shifting Habitat for One Species}

As an extension study for spatial population ecology, our analysis is based on reaction-diffusion models like Fisher's equation and discusses the effect the rate of climate change for the persistence and spread of species. The model is given by

$$
\frac{\partial u}{\partial t}=d \frac{\partial^{2} u}{\partial x^{2}}+u r(x-c t)-u^{2}
$$

Here $u$ is the population density of the species at time $t$ and position $-\infty<x<\infty$, $d$ is a constant diffusion coefficient, $r(x-c t)$ represents the population growth rate, and $-u^{2}$ denotes the density dependent death.

We assume that $r(x)$ is continuous and nondecreasing and bounded from below by $r(-\infty)$ and above by $r(\infty)>0$. The monotonicity of $r(x)$ in $x$ reflects that the quality of habitat improves to the right along the $x$-axis. The assumption $r(x) \leq$ $r(\infty)$ indicates that the population growth rate is limited by the maximum carry capacity of the environment. We use the function $r(x-c t)$ to represent the habitat shifting with climate change, where $c$ is a real number. The persistence and spread of species along shifting habitat had been studied (Li et al. 2015) assuming $r(-\infty)<0$, which means, to the left of the region of poor quality, the species cannot grow. In this Chapter, we assume that when $r(-\infty)>0$, the species can grows everywhere along the spatial gradient, but with differing levels of success. The potential domain of the species is unbounded and can be distinguishable as a higher quality region with higher population growth rate (favorable habitat) and a lower quality region with lower population growth rate (less favorable habitat). The edge of the favorable habitat for species is shifting at speed $c$. When $c$ is positive, the function $r(x-c t)$ implies that the resource distribution propagates rightward at speed $c$, so that the habitat with better quality contracts. When $c$ is negative, this function implies that the resource distribution propagates leftward at speed $|c|$, so that the habitat with better quality expands. We are interested in the spread and persistence of the species 
for different $c$ values.

We make the following hypothesis for $r(x)$ for our mathematical analysis:

Hypothesis II.1 $r(x)$ is nondecreasing, bounded, and piecewise continuously differentiable function in $x$ for $-\infty<x<\infty, 0<r(-\infty)<r(\infty)<\infty$.

We recall the classical definitions of upper and lower solutions corresponding to (3) in Pao (1992).

Definition II.2 A function $\tilde{u}(t, x)$ with $t>0$ and $x \in \mathrm{R}$ is called an upper solution of (3) if the following inequality is satisfied:

$$
\frac{\partial \tilde{u}}{\partial t} \geq d \frac{\partial^{2} \tilde{u}}{\partial x^{2}}+\tilde{u} r(x-c t)-\tilde{u}^{2} .
$$

Similarly, $\hat{u}(t, x)$ is called a lower solution if the reversed inequality in (4) is satisfied.

The functions $\tilde{u}(t, x)$ and $\hat{u}(t, x)$ are called ordered upper and lower solutions if $\tilde{u}(t, x) \geq \hat{u}(t, x)$ for all $t>0$ and $-\infty<x<\infty$. From the definition, $\tilde{u}(t, x)=r(\infty)$ and $\hat{u}(t, x)=0$ are ordered upper and lower solutions of (3). To explore the existence of a solution to (3), we need to verify some necessary conditions on the reaction function. A basic assumption on $f(u, t, x)=u r(x-c t)-u^{2}$ is the following one-sided Lipschitz condition

$$
\begin{aligned}
f\left(u_{1}, t, x\right)-f\left(u_{2}, t, x\right) & =u_{1} r(x-c t)-u_{1}^{2}-\left(u_{2} r(x-c t)-u_{2}^{2}\right) \\
& =\left(u_{1}-u_{2}\right)\left(r(x-c t)-u_{1}-u_{2}\right) \\
& \geq-\rho\left(u_{1}-u_{2}\right),
\end{aligned}
$$

for $0 \leq u_{2} \leq u_{1} \leq r(\infty)$ and $\rho$ is a constant. It is easy to prove that $u r(x-c t)-u^{2}$ is Lipschitz continuous with $\rho>3 r(\infty)$, then the function

$$
\mathrm{F}(u, t, x) \equiv u(\rho+r(x-c t)-u)
$$

is nondecreasing in $u$ for $0 \leq u \leq r(\infty)$. By adding a dominant linear term $\rho u(t, x)$ to both sides of (3), we obtain the equivalent equation of (3):

$$
\frac{\partial u}{\partial t}+\rho u=d \frac{\partial^{2} u}{\partial x^{2}}+\rho u+u r(x-c t)-u^{2} .
$$


Obviously, $u \equiv 0$ is a trivial solution and $u \equiv r(\infty)$ is an upper solution of (5). From Pao (1992), the solution, $u(t, x)$, of model (5) with $u(0, x)=u_{0}(x)$, where $u_{0}(x)$ is continuous in $x$ and $0 \leq u_{0}(x) \leq r(\infty)$, can be expressed as the fixed point of the nonlinear integral equation as follows,

$$
\begin{aligned}
u(t, x)= & \int_{-\infty}^{+\infty} k(t, x-y) u_{0}(y) d y+ \\
& \int_{0}^{t} \int_{-\infty}^{+\infty} k(t-\tau, x-y) u(\tau, y)[\rho+r(y-c \tau)-u(\tau, y)] d y d \tau,
\end{aligned}
$$

where

$$
k(t, y)=\frac{1}{\sqrt{4 \pi d t}} e^{-\rho t-\frac{y^{2}}{4 d t}}
$$

and

$$
\int_{-\infty}^{+\infty} k(t, y) d y=e^{-\rho t}
$$

Consider the sequence $u^{(n)}(t, x)$ generated by:

$$
\begin{aligned}
u^{(n+1)}(t, x)= & Q\left[u^{(n)}\right](t, x)=\int_{-\infty}^{+\infty} k(t, x-y) u_{0}(y) d y+ \\
& \int_{0}^{t} \int_{-\infty}^{+\infty} k(t-\tau, x-y) u^{(n)}(\tau, y)\left[\rho+r(y-c \tau)-u^{(n)}(\tau, y)\right] d y d \tau,
\end{aligned}
$$

where $u^{(0)}(t, x)=0$ or $u^{(0)}(t, x)=r(\infty)$. Lemma 7.22 in Pao (1992) shows that the sequence $u^{(n)}(t, x)$ is nondecreasing in $n$ if $u^{(0)}(t, x)=r(-\infty)$ and nonincreasing in $n$ if $u^{(0)}(t, x)=r(\infty)$. Lemma 7.22 shows in both cases $u(t, x)=\lim _{n \rightarrow \infty} u^{(n)}(t, x)$ is the solution of $(5)$ with $u(0, x)=u_{0}(x)$, and $0 \leq u(t, x) \leq r(\infty)$. Theorem 2.1 in Pao (1992) and Lemma 1.2 in Wang (1993) show the existence and uniqueness of the solution $u(t, x)$ for model (5). As an application of Theorem 2.1 in Pao (1992), we present below the comparison principle corresponding to the reaction-diffusion equation, which will be the principal tool in subsequent discussions.

Lemma II.1 (Comparison Principle) If $u^{(n)}(t, x)$ and $v^{(n)}(t, x)$ are two sequences of continuous and nonnegative functions with the properties $v^{(n+1)}(t, x) \leq Q\left[v^{(n)}\right](t, x)$ and $u^{(n+1)}(t, x) \geq Q\left[u^{(n)}\right](t, x)$ for all nonnegative $n$ and $v^{(0)}(t, x) \leq u^{(0)}(t, x) \leq r(\infty)$, then $v^{(n)}(t, x) \leq u^{(n)}(t, x) \leq r(\infty)$ for all positive integers $n$. 
Proof. We assume that $v^{(n-1)}(t, x) \leq u^{(n-1)}(t, x) \leq r(\infty)$ for some positive integers $n$. Since $Q[u]$ is nondecreasing in $u, v^{(n+1)}(t, x) \leq Q\left[v^{(n)}\right](t, x)$ and $u^{(n+1)}(t, x) \geq Q\left[u^{(n)}\right](t, x)$ for all nonnegative $n$, we have that

$$
v^{(n)}(t, x) \leq Q\left[v^{(n-1)}\right](t, x) \leq Q\left[u^{(n-1)}\right](t, x) \leq u^{(n)}(t, x)
$$

Since $v^{(0)}(t, x) \leq u^{(0)}(t, x) \leq r(\infty)$, by induction, $v^{(n)}(t, x) \leq u^{(n)}(t, x) \leq r(\infty)$ is valid for all positive integers $n$.

To consider the spreading speed of the reaction-diffusion equation, we introduce some notations (Weinberger 1982; Li et al. 2015). Define

$$
\phi(x ; \mu)=\frac{d \mu^{2}+r(x)}{\mu} \text { and } \quad \psi(\mu)=2 d \mu .
$$

Observe that $\psi(\mu)$ is a strictly increasing function for $\mu>0$ and $\phi(x ; \mu)$ is strictly decreasing for $0 \leq \mu \leq \mu^{*}(x)$ at each number $x$. We have that $\phi(x ; \mu)>\psi(\mu)$ for $0<\mu<\mu^{*}(x)$. Furthermore, $\phi(x ; \mu)$ intersects with $\psi(\mu)$ at the coordinate where the infimum of $\phi(x ; \mu)$ is attained.

From Li et al. (2015), the spreading speed

$$
c^{*}(x)=\inf _{\mu>0} \phi(x ; \mu)=2 \sqrt{d r(x)}
$$

which is the only minimum of $\phi(x ; \mu)$. Let $\mu=\mu^{*}(x)=\sqrt{\frac{r(x)}{d}}$ be the unique point where the minimum occurs, i.e.,

$$
c^{*}(x)=\phi\left(x ; \mu^{*}(x)\right) .
$$

It follows that, when $r(\infty)>r(-\infty)>0$,

$$
c^{*}(\infty)=2 \sqrt{d r(\infty)} \text { and } c^{*}(-\infty)=2 \sqrt{d r(-\infty)}
$$

so that

$$
c^{*}(\infty) \geq c^{*}(-\infty)
$$

We will demonstrate that under appropriate conditions, the rightward spreading speed is given by $c^{*}(\infty)$ or $c^{*}(-\infty)$, and the leftward spreading speed is given by $c^{*}(\infty)$, $c^{*}(-\infty)$ or $|c|$. 


\section{Upper Solutions}

In this section, we discuss the upper solutions for model (5). When $c=0$, we provide a lemma for the equation

$$
\frac{\partial u}{\partial t}=d \frac{\partial^{2} u}{\partial x^{2}}+r(x) u-u^{2}
$$

which is essentially Lemma 2.1 in Li et al. (2015).

Lemma II.2 Let $\bar{u}(t, x)$ be the solution of model (9) with $\bar{u}(0, x)=r(\infty)$. Then $\bar{u}(t, x)$ is nonincreasing in $t$ and nondecreasing in $x, \bar{u}(t,-\infty)=r(-\infty)$, and $\bar{u}(t, \infty)=$ $r(\infty)$ for $t>0$.

The proof of this lemma is similar to the one of the Lemma 2.1 in $\mathrm{Li}$ et al. (2015), so we omit the details.

Lemma II.3 Let $u(t, x)$ be the solution of (5) with $u(0, x)=u_{0}(x)$, where $0 \leq$ $u_{0}(x) \leq r(\infty)$. For any $\epsilon>0$, there exists sufficiently large $T>0$ and $M>0$, such that, when $t>T$ and $x \leq-M+c t$,

$$
u(t, x) \leq r(-\infty)+\epsilon
$$

Proof. Firstly, we consider the case of $c \geq 0$. For any $\epsilon>0$, we set

$$
\epsilon_{0}=\left(\sqrt{(4+r(-\infty))^{2}+4 \epsilon}-4-r(-\infty)\right) / 2>0
$$

Let $w(t, x)=u(t, x)-r(-\infty)-\epsilon_{0}$. Since $u(t, x)$ is the solution of (5) with $u(0, x)=$ $u_{0}(x), w(t, x)$ satisfies the following equation:

$$
\frac{\partial w}{\partial t}=d \frac{\partial^{2} w}{\partial x^{2}}+\left(w+r(-\infty)+\epsilon_{0}\right)\left(r(x-c t)-w-r(-\infty)-\epsilon_{0}\right),
$$

which is equivalent to:

$$
\begin{aligned}
\frac{\partial w}{\partial t}+\rho w= & d \frac{\partial^{2} w}{\partial x^{2}}+\rho w+\left(w+r(-\infty)+\epsilon_{0}\right)\left(r(x-c t)-w-r(-\infty)-\epsilon_{0}\right) \\
= & d \frac{\partial^{2} w}{\partial x^{2}}+\left(w+r(-\infty)+\epsilon_{0}\right)\left(\rho+r(x-c t)-w-r(-\infty)-\epsilon_{0}\right) \\
& -\rho\left(r(-\infty)+\epsilon_{0}\right),
\end{aligned}
$$


with $\rho>3 r(\infty)$. The solution of the equation (10) with initial value $w(0, x)=$ $u(0, x)-r(-\infty)-\epsilon_{0}$ satisfies the integral equation

$$
\begin{aligned}
w(t, x) & =\int_{-\infty}^{+\infty} e^{-\rho t} h(t, x-y) w(0, y) d y+ \\
& \int_{0}^{t} \int_{-\infty}^{+\infty} e^{-\rho \tau} h(\tau, y)\left[\rho w(t-\tau, x-y)+\left(w(t-\tau, x-y)+r(-\infty)+\epsilon_{0}\right) .\right. \\
& \left.\left(r(x-c t+c \tau-y)-w(t-\tau, x-y)-r(-\infty)-\epsilon_{0}\right)\right] d y d \tau,
\end{aligned}
$$

where

$$
h(\tau, y)=\frac{1}{\sqrt{4 \pi d \tau}} e^{-\frac{y^{2}}{4 d \tau}} .
$$

Since $0 \leq u(t, x) \leq r(\infty)$, it is easy to see that the following expression is bounded:

$$
\begin{aligned}
& \mid \rho w(t-\tau, x-y)+\left(w(t-\tau, x-y)+r(-\infty)+\epsilon_{0}\right) . \\
& \left(r(x-c t+c \tau-y)-w(t-\tau, x-y)-r(-\infty)-\epsilon_{0}\right) \mid \\
& \leq r(\infty)(\rho+2 r(\infty))+\rho\left(r(-\infty)+\epsilon_{0}\right) .
\end{aligned}
$$

Therefore,

$$
\begin{aligned}
& \int_{0}^{\infty} \int_{-\infty}^{+\infty} e^{-\rho \tau} h(\tau, y)\left[\rho w(t-\tau, x-y)+\left(w(t-\tau, x-y)+r(-\infty)+\epsilon_{0}\right)\right. \\
& \left.\left(r(x-c t+c \tau-y)-w(t-\tau, x-y)-r(-\infty)-\epsilon_{0}\right)\right] d y d \tau \\
& \leq \int_{0}^{\infty} \int_{-\infty}^{+\infty} e^{-\rho \tau} h(\tau, y)\left[r(\infty)(\rho+2 r(\infty))+\rho\left(r(-\infty)+\epsilon_{0}\right)\right] d y d \tau \\
& \leq \int_{0}^{\infty} e^{-\rho \tau} d \tau\left[r(\infty)(\rho+2 r(\infty))+\rho\left(r(-\infty)+\epsilon_{0}\right)\right] \\
& \leq \frac{r(\infty)(\rho+2 r(\infty))+\rho\left(r(-\infty)+\epsilon_{0}\right)}{\rho}
\end{aligned}
$$

It follows that, for the given $\epsilon_{0}>0$, there exists $\eta>0$ and $A>\eta$, such that

$$
\begin{aligned}
& \int_{0}^{\eta} \int_{-\infty}^{+\infty} e^{-\rho \tau} \mid h(\tau, y)\left[\rho w(t-\tau, x-y)+\left(w(t-\tau, x-y)+r(-\infty)+\epsilon_{0}\right) .\right. \\
& \left.\left(r(x-c t+c \tau-y)-w(t-\tau, x-y)-r(-\infty)-\epsilon_{0}\right)\right] \mid d y d \tau<\epsilon_{0}
\end{aligned}
$$

and

$$
\begin{aligned}
& \int_{A}^{\infty} \int_{-\infty}^{+\infty} e^{-\rho \tau} \mid h(\tau, y)\left[\rho w(t-\tau, x-y)+\left(w(t-\tau, x-y)+r(-\infty)+\epsilon_{0}\right) .\right. \\
& \left.\left(r(x-c t+c \tau-y)-w(t-\tau, x-y)-r(-\infty)-\epsilon_{0}\right)\right] \mid d y d \tau<\epsilon_{0} .
\end{aligned}
$$


For $m>0$, let

$$
\begin{aligned}
& \int_{\eta}^{A} \int_{-\infty}^{+\infty} e^{-\rho \tau} \mid h(\tau, y)\left[\rho w(t-\tau, x-y)+\left(w(t-\tau, x-y)+r(-\infty)+\epsilon_{0}\right)\right. \\
& \left.\left(r(x-c t+c \tau-y)-w(t-\tau, x-y)-r(-\infty)-\epsilon_{0}\right)\right] \mid d y d \tau \\
& =I(m)+I I(m)
\end{aligned}
$$

where

$$
\begin{aligned}
& I(m)=\int_{\eta}^{A} \int_{-\infty}^{c \tau-m} e^{-\rho \tau} \mid h(\tau, y)\left[\rho w(t-\tau, x-y)+\left(w(t-\tau, x-y)+r(-\infty)+\epsilon_{0}\right) .\right. \\
& \left.\left(r(x-c t+c \tau-y)-w(t-\tau, x-y)-r(-\infty)-\epsilon_{0}\right)\right] \mid d y d \tau
\end{aligned}
$$

and

$$
\begin{aligned}
& I I(m)=\int_{\eta}^{A} \int_{c \tau-m}^{\infty} e^{-\rho \tau} \mid h(\tau, y)\left[\rho w(t-\tau, x-y)+\left(w(t-\tau, x-y)+r(-\infty)+\epsilon_{0}\right) .\right. \\
& \left.\left(r(x-c t+c \tau-y)-w(t-\tau, x-y)-r(-\infty)-\epsilon_{0}\right)\right] \mid d y d \tau .
\end{aligned}
$$

Changing the variable $y$ to be $z=\frac{y}{\sqrt{4 d \tau}}$, we have that

$$
\begin{aligned}
& I(m) \leq \int_{\eta}^{A} \int_{-\infty}^{(c \tau-m) / \sqrt{4 d \tau}} e^{-\rho \tau} \frac{1}{\pi} e^{-z^{2}} \mid \rho w(t-\tau, x-\sqrt{4 d \tau} z)+(w(t-\tau, x-\sqrt{4 d \tau} z) \\
& \left.\left.+r(-\infty)+\epsilon_{0}\right) \cdot\left(r(x-c t+c \tau-y)-w(t-\tau, x-\sqrt{4 d \tau} z)-r(-\infty)-\epsilon_{0}\right)\right] \mid d y d \tau .
\end{aligned}
$$

Since $(c \tau-m) / \sqrt{4 d \tau}$ uniformly converges to $-\infty$ for any $\tau \in[\eta, A]$ as $m \rightarrow \infty$, and $\mid \rho w(t-\tau, x-\sqrt{4 d \tau} z)+\left(w(t-\tau, x-\sqrt{4 d \tau} z)+r(-\infty)+\epsilon_{0}\right)(r(x-c t+c \tau-y)-w(t-$ $\left.\left.\tau, x-\sqrt{4 d \tau} z)-r(-\infty)-\epsilon_{0}\right)\right] \mid$ is bounded, for the given $\epsilon_{0}$, there exists sufficiently large $m_{0}$ such that

$$
I\left(m_{0}\right)<\epsilon_{0}
$$

Now we are going to prove $I I\left(m_{0}\right)<\epsilon_{0}(r(-\infty)+\epsilon)$.

By the monotonicity of $r(x)$, there is sufficiently large $M_{1}>0$, such that for $x<-M_{1}+c t$

$$
r\left(x-c t+m_{0}\right)<r(-\infty)+\epsilon_{0}
$$

From Lemma II.2, $\bar{u}(t, x)$ is nonincreasing in $t$ and nondecreasing in $x, \bar{u}(t,-\infty)=$ $r(-\infty)$ and $\bar{u}(t, \infty)=r(\infty)$ for $t>0$. It follows that, for the given $\epsilon_{0}$, there are sufficiently large $T_{2}>0$ and $M_{2}>0$, such that for $t>A+T_{2}$, and $x<-M_{2}$,

$$
\bar{u}\left(t-A, x-c A+m_{0}\right)<r(-\infty)+\epsilon_{0} .
$$


By the definition of $I I\left(m_{0}\right)$, its integration variables $\tau$ and $y$ satisfy $c \tau-y \leq m_{0}$.

We have that

$$
\begin{aligned}
I I\left(m_{0}\right) \quad & =\int_{\eta}^{A} \int_{c \tau-m_{0}}^{\infty} e^{-\rho \tau} \mid h(\tau, y)[u(t-\tau, x-y) \\
& \left.\left(\rho+r\left(x-c t+m_{0}\right)-u(t-\tau, x-y)\right)-\rho(r(-\infty)+\epsilon)\right] \mid d y d \tau \\
& \leq \int_{\eta}^{A} \int_{c \tau-m_{0}}^{\infty} e^{-\rho \tau} \mid h(\tau, y)[\bar{u}(t-\tau, x-y) \\
& \left.\left(\rho+r\left(x-c t+m_{0}\right)-\bar{u}(t-\tau, x-y)\right)-\rho(r(-\infty)+\epsilon)\right] \mid d y d \tau \\
& \leq \int_{\eta}^{A} \int_{c \tau-m_{0}}^{\infty} e^{-\rho \tau} \mid h(\tau, y)\left[\bar{u}\left(t-A, x-c A+m_{0}\right)\right. \\
& \left.\left(\rho+r\left(x-c t+m_{0}\right)-r(-\infty)\right)-\rho\left(r(\infty)+\epsilon_{0}\right)\right] \mid d y d \tau \\
& \leq \int_{\eta}^{A} \int_{c \tau-m_{0}}^{\infty} e^{-\rho \tau}\left|h(\tau, y)\left[\left(r(-\infty)+\epsilon_{0}\right)\left(\rho+\epsilon_{0}\right)-\rho\left(r(\infty)+\epsilon_{0}\right)\right]\right| d y d \tau \\
& =\left(r(-\infty)+\epsilon_{0}\right) \epsilon_{0} \int_{\eta}^{A} e^{-\rho \tau} d \tau \\
& \leq\left(r(-\infty)+\epsilon_{0}\right) \epsilon_{0} .
\end{aligned}
$$

Since $|w(0, x)|=\left|u(0, x)-r(-\infty)-\epsilon_{0}\right|<r(\infty)-r(-\infty)-\epsilon_{0}$,

$$
\int_{-\infty}^{+\infty} e^{-\rho t} h(t, x-y) w(0, y) d y \leq\left(r(\infty)-r(-\infty)-\epsilon_{0}\right) e^{-\rho t}
$$

We have that, for the given $\epsilon_{0}$, there exists $T_{3}>0$, such that for $t>T_{3}$,

$$
\int_{-\infty}^{+\infty} e^{-\rho t} h(t, x-y) w(0, y) d y \leq \epsilon_{0}
$$

Therefore, for $t \geq T:=\max \left\{T_{2}, T_{3}\right\}$ and $x \leq-M+c t$ with $M:=\max \left\{M_{1}, M_{2}\right\}$,

$$
w(t, x) \leq \epsilon_{0}\left(4+r(-\infty)+\epsilon_{0}\right)=\epsilon,
$$

which implies that

$$
u(t, x) \leq r(-\infty)+\epsilon .
$$

In this case of $c<0$, we assume $\bar{u}(t, x)$ to be the solution of model (9) with $\bar{u}(0, x)=r(\infty)$. From Lemma II.2, $\bar{u}(t,-\infty)=r(-\infty)$. Then for any $\epsilon>0$, there exists $T_{1}>0$ and $M>0$ such that for $x<-M, \bar{u}\left(T_{1}, x\right)<r(-\infty)+\epsilon$. Since $\bar{u}(t, x)$ is nonincreasing in $t$, we have that

$$
\bar{u}(t, x)<r(-\infty)+\epsilon \text { for } t \geq T_{1} \text { and } x \leq-M .
$$


The function $\check{u}:=\bar{u}(t, x-c t)$ satisfies

$$
\frac{\partial \check{u}}{\partial t}=d \frac{\partial^{2} \check{u}}{\partial x^{2}}-c \frac{\partial \check{u}}{\partial x}+\check{u}(r(x-c t)-\check{u}) .
$$

Since $c<0$, we have that

$$
\frac{\partial \check{u}}{\partial t} \geq d \frac{\partial^{2} \check{u}}{\partial x^{2}}+\check{u}(r(x-c t)-\check{u})
$$

So $\check{u}(t, x)$ is an upper solution of model (3). Let $u(0, x) \leq r(\infty)$, we have that $u(t, x) \leq \check{u}(t, x)$. It yields that

$$
u(t, x)<r(-\infty)+\epsilon \text { for } t \geq T_{1} \text { and } x \leq-M+c t
$$

The proof is complete.

We introduce the following lemma regarding two upper solutions for model (3).

Lemma II.4 Assume that $u(t, x)$ is a solution of model (3).

(a) If $u(0, x)$ is zero for all sufficiently large $x$, and $0 \leq u(0, x) \leq r(\infty)$, then for any small positive number $\epsilon$ there exist positive numbers $A$ and $\mu_{\epsilon}$ such that

$$
u(t, x) \leq A e^{-\mu_{\epsilon}\left(x-\left(c^{*}(\infty)+\epsilon\right) t\right)}
$$

(b) Assume $c>-c^{*}(-\infty)$. If $u(0, x)$ is zero for all sufficiently negative $x$, and $0 \leq u(0, x) \leq r(\infty)$, then for any positive $\epsilon_{1}$, there exist positive numbers $B$ and $\mu_{1}$ such that for all $t>0$ and $-\infty<x<\infty$,

$$
u(t, x) \leq w(t, x)=B e^{\mu_{1}\left(x+\left(c^{*}(-\infty)+\epsilon_{1}\right) t\right)} .
$$

Proof. We first prove the statement (a). For any $\epsilon>0$, since $\phi(x ; \mu)$ decreases in $0<\mu<\mu^{*}(x)$, there exist $\mu_{\epsilon}$ such that $\phi\left(\infty ; \mu_{\epsilon}\right)=c^{*}(\infty)+\epsilon$. We assume $A$ is a positive number and

$$
\hat{u}(t, x)=A e^{-\mu_{\epsilon}\left(x-\left(c^{*}(\infty)+\epsilon\right) t\right)} .
$$

It is easy to show that $\hat{u}(t, x)$ is a solution of

$$
\frac{\partial u}{\partial t}=d \frac{\partial^{2} u}{\partial x^{2}}+r(\infty) u
$$


Since $r(\infty) u \geq u(r(x-c t)-u), \hat{u}(t, x)$ is an upper solution of model (3). Choose $A$ sufficiently large such that $u(0, x) \leq \hat{u}(0, x)=A e^{-\mu_{\epsilon} x}$. Then

$$
u(t, x) \leq \hat{u}(t, x)=A e^{-\mu_{\epsilon}\left(x-\left(c^{*}(\infty)+\epsilon\right) t\right)} .
$$

We now prove the statement (b). By the definition of $c^{*}(x)$, we have that

$$
c^{*}(-\infty)=\inf _{\mu>0} \frac{d \mu^{2}+r(-\infty)}{\mu},
$$

or equivalently,

$$
c^{*}(-\infty)=\lim _{\delta \rightarrow 0} \inf _{\mu>0} \frac{d \mu^{2}+r(-\infty)+\delta}{\mu} .
$$

Therefore, for any $\epsilon_{1}>0$, there exists $\delta>0$ and $0<\mu_{1}<\mu^{*}(-\infty)$, such that

$$
c^{*}(-\infty)+\epsilon_{1}=\frac{d \mu_{1}^{2}+r(-\infty)+\delta}{\mu_{1}} .
$$

It follows that

$$
\mu_{1}\left(c^{*}(-\infty)+\epsilon_{1}\right)=d \mu_{1}^{2}+r(-\infty)+\delta .
$$

Since $r(x)$ is continuous and increasing in $-\infty<x<\infty$, there exists $x_{1}$ such that if $x<x_{1}$, then

$$
r(x) \leq r(-\infty)+\delta .
$$

It follows that for any $t>0$, if $x<x_{1}+c t$ then

$$
r(x-c t) \leq r(-\infty)+\delta .
$$

Additionally, there exists $B$ sufficiently large such that $B e^{\mu_{1} x_{1}}=r(\infty)$.

Let

$$
S[u](t, x)=\frac{\partial u}{\partial t}-d \frac{\partial^{2} u}{\partial x^{2}}-r(x-c t) u+u^{2},
$$

then

$$
\begin{aligned}
S[w](t, x) & =\frac{\partial w}{\partial t}-d \frac{\partial^{2} u}{\partial x^{2}}-r(x-c t) w+w^{2} \\
& =w(t, x)\left[\mu_{1}\left(c^{*}(-\infty)+\epsilon_{1}\right)-d \mu_{1}^{2}-r(x-c t)+w(t, x)\right] \\
& =w(t, x)\left[r(-\infty)+\delta-r(x-c t)+B e^{\mu_{1}\left(x+\left(c^{*}(-\infty)+\epsilon_{1}\right) t\right)}\right] .
\end{aligned}
$$


It follows that for any $t>0$, when $x<x_{1}+c t$, we have that

$$
S[w](t, x) \geq w(t, x) B e^{\mu_{1}\left(x+\left(c^{*}(-\infty)+\epsilon_{1}\right) t\right)}>0,
$$

and when $x>x_{1}+c t$, we have that,

$$
B e^{\mu_{1}\left(x+\left(c^{*}(-\infty)+\epsilon_{1}\right) t\right)} \geq B e^{\mu_{1}\left(x_{1}+c t+\left(c^{*}(-\infty)+\epsilon_{1}\right) t\right)} \geq B e^{\mu_{1} x_{1}}=r(\infty) .
$$

So $S[w](t, x)>0$ for any $-\infty<x<\infty$ and $t>0$.

Therefore, $w(t, x)$ is an upper solution of $u(t, x)$, i.e.

$$
u(t, x) \leq B e^{\mu_{1}\left(x+\left(c^{*}(-\infty)+\epsilon_{1}\right) t\right)} .
$$

The proof is complete.

The next lemma is necessary in proving the theorems in section 5 .

Lemma II.5 Assume that $c$ and $\mu$ are constants. For any $\epsilon>0$ sufficiently small, there exist $b>0$ sufficiently large and $S_{0}$ a positive number such that for any real number $x$,

$$
\begin{aligned}
& \int_{0}^{t} \int_{-\infty}^{\infty} k(\tau, y) e^{\mu y} r(x-y-c(t-\tau)) d y d \tau \\
& \leq\left(1+S_{0} \epsilon\right) \int_{0}^{t} \int_{c \tau-b}^{\infty} k(\tau, y) e^{\mu y} r(x-y-c(t-\tau)) d y d \tau
\end{aligned}
$$

where $k(\tau, y)$ is defined by the formula (6).

Proof. Choose $\eta, A$ and $m$ to be any positive numbers and $\rho$ satisfying $\rho>d \mu^{2}$.

Let

$$
\int_{0}^{t} \int_{-\infty}^{\infty} k(\tau, y) e^{\mu y} d y d \tau=\mathrm{I}(\eta)+\mathrm{I}(A)+\mathrm{I}(m)+\mathrm{II}(m)
$$

where

$$
\begin{aligned}
\mathrm{I}(A) & =\int_{A}^{\infty} \int_{-\infty}^{\infty} k(\tau, y) e^{\mu y} r(x-y-c(t-\tau)) d y d \tau, \\
\mathrm{I}(\eta) & =\int_{0}^{\eta} \int_{-\infty}^{\infty} k(\tau, y) e^{\mu y} r(x-y-c(t-\tau)) d y d \tau, \\
\mathrm{I}(m) & =\int_{\eta}^{A} \int_{-\infty}^{c \tau-m} k(\tau, y) e^{\mu y} r(x-y-c(t-\tau)) d y d \tau, \\
\mathrm{II}(m) & =\int_{\eta}^{A} \int_{c \tau-m}^{\infty} k(\tau, y) e^{\mu y} r(x-y-c(t-\tau)) d y d \tau .
\end{aligned}
$$


Here we have omitted explicitly listing the x dependency of $I$, and $I I$. Since

$$
\begin{aligned}
\mathrm{I}(A) & \leq \int_{A}^{\infty} \int_{-\infty}^{\infty} k(\tau, y) e^{\mu y} r(\infty) d y d \tau \\
& =r(\infty) \int_{A}^{\infty} e^{-\rho \tau+d \tau \mu^{2}} \int_{-\infty}^{\infty} \frac{1}{\sqrt{4 \pi d \tau}} e^{-\frac{(y-2 d \tau \mu)^{2}}{4 d \tau}} d y d \tau \\
& =r(\infty) \int_{A}^{\infty} e^{-\rho \tau+d \tau \mu^{2}} d y d \tau \\
& =\frac{r(\infty)}{\rho-d \mu^{2}} e^{-\left(\rho-d \mu^{2}\right) A}
\end{aligned}
$$

and

$$
\mathrm{I}(\eta) \leq \int_{0}^{\eta} \int_{-\infty}^{\infty} k(\tau, y) e^{\mu y} r(\infty) d y d \tau=\frac{r(\infty)}{\rho-d \mu^{2}}\left(1-e^{-\left(\rho-d \mu^{2}\right) \eta}\right)
$$

for any positive $\epsilon$, there exist $\eta$ sufficiently small and $A$ sufficiently large, such that $\mathrm{A}(\eta)<\frac{\epsilon}{4}$ and $\mathrm{I}(\eta)<\frac{\epsilon}{4}$.

Changing the variable $y$ to $z=(y-2 d \tau \mu) / \sqrt{4 d \tau}$ in $\mathrm{I}(m)$, we have that

$$
\begin{aligned}
\mathrm{I}(m) & \leq \int_{\eta}^{A} \int_{-\infty}^{c \tau-m} k(\tau, y) e^{\mu y} r(\infty) d y d \tau \\
& =r(\infty) \int_{\eta}^{A} e^{-\rho \tau+d \tau \mu^{2}} \int_{-\infty}^{c \tau-m} \frac{1}{\sqrt{4 \pi d \tau}} e^{-\frac{(y-2 d \tau \mu)^{2}}{4 d \tau}} d y d \tau \\
& =r(\infty) \int_{\eta}^{A} e^{-\rho \tau+d \tau \mu^{2}} \int_{-\infty}^{\frac{c \tau-2 d \mu \tau-m}{\sqrt{4 d \tau}}} \frac{1}{\sqrt{\pi}} e^{-z^{2}} d y d \tau
\end{aligned}
$$

Since $\frac{c \tau-2 d \mu \tau-m}{\sqrt{4 d \tau}} \rightarrow-\infty$ uniformly for $\tau \in[\eta, A]$ as $m \rightarrow \infty$, for the given $\epsilon>0$, there exists sufficiently large $m=b$ such that

$$
\mathrm{I}(b)<\frac{\epsilon}{4}
$$

Additionally,

$$
\begin{aligned}
\mathrm{II}(b) & \leq \int_{\eta}^{A} \int_{c \tau-b}^{\infty} k(\tau, y) e^{\mu y} r(\infty) d y d \tau \\
& =r(\infty) \int_{\eta}^{A} e^{-\rho \tau+d \tau \mu^{2}} \int_{c \tau-b}^{\infty} \frac{1}{\sqrt{4 \pi d \tau}} e^{-\frac{(y-2 d \tau \mu)^{2}}{4 d \tau}} d y d \tau \\
& \leq r(\infty) \int_{\eta}^{A} e^{-\rho \tau+d \tau \mu^{2}} d \tau \\
& =\frac{r(\infty)}{\rho-d \mu^{2}}\left(e^{-\left(\rho-d \mu^{2}\right) \eta}-e^{-\left(\rho-d \mu^{2}\right) A}\right) .
\end{aligned}
$$


Similarly, we can prove that

$$
\begin{aligned}
\mathrm{II}(b) & \geq \int_{\eta}^{A} \int_{c \tau-b}^{\infty} k(\tau, y) e^{\mu y} r(-\infty) d y d \tau \\
& \geq \frac{r(-\infty)}{\rho-d \mu^{2}}\left(e^{-\left(\rho-d \mu^{2}\right) \eta}-e^{-\left(\rho-d \mu^{2}\right) A}\right)-\frac{r(-\infty)}{r(\infty)} \frac{\epsilon}{2}
\end{aligned}
$$

It follows that $\mathrm{II}(b)$ is positive and bounded. For the given $\epsilon$, there exists a positive number $S_{0}$ such that

$$
\mathrm{I}(A)+\mathrm{I}(\eta)+\mathrm{I}(b)<S_{0} \epsilon \mathrm{II}(b)
$$

Therefore,

$$
\begin{aligned}
& \int_{0}^{t} \int_{-\infty}^{\infty} k(\tau, y) e^{\mu y} r(x-y-c(t-\tau)) d y d \tau \\
& \leq\left(1+S_{0} \epsilon\right) \int_{0}^{t} \int_{c \tau-b}^{\infty} k(\tau, y) e^{\mu y} r(x-y-c(t-\tau)) d y d \tau
\end{aligned}
$$

The proof is complete.

\section{Lower Solutions}

Now we construct lower solutions for model (5) by extending the work in Li et al. (2015). We provide a definition of a weak lower solution which was introduced in Li et al. (2015) and is also a modified version of definition 1.1 in Wang (1993).

Definition II.3 We call a function u a continuous weak lower solution of model (3) if $u$ is continuous for $t \geq 0$ and $-\infty<x<\infty$, and

$$
\frac{\partial u}{\partial t} \leq d \frac{\partial^{2} u}{\partial x^{2}}+u r(x-c t)-u^{2}
$$

in the distributional sense, i.e., for any $T>0$ and any $\eta \in \mathrm{C}^{2,1}((-\infty, \infty) \times[0, T])$ with $\eta \geq 0$ and $\operatorname{supp} \eta(\cdot, t)$ being a bounded interval for all $t \in[0, T]$,

$$
\begin{aligned}
& \left.\int_{-\infty}^{\infty} u(t, x) \eta(t, x) d x\right|_{t=0} ^{t=T_{1}} \\
& \leq \int_{0}^{T_{1}} \int_{-\infty}^{\infty}\left[u(s, x)\left(d \eta_{x x}+\eta_{t}\right)(s, x)+\eta(s, x) u(s, x)(r(x-c s)-u(s, x))\right] d x d s
\end{aligned}
$$

if $T_{1} \in[0, T]$. 
We recall the function $v(\mu ; x)$ to construct the lower solution of system $(3)$, which was used in Li et al. (2015). The function $v(\mu ; x)$ is given by

$$
v(\mu ; x)= \begin{cases}e^{-\mu x} \sin \gamma x, & \text { if } 0 \leq x \leq \frac{\pi}{\gamma} \\ 0, & \text { elsewhere }\end{cases}
$$

with $\gamma>0$ and $\eta>0$. This function was initially introduced in Weinberger (1982). It is easy to see that $v(\mu ; x)$ is continuous and second order derivative in $x$ except at the point $x=0$ and $x=\frac{\pi}{\gamma}$. The maximum of $v(\mu ; x)$ occurs at $\sigma(\mu)=\frac{1}{\gamma} \tan ^{-1}\left(\frac{\gamma}{\mu}\right)$. The function $\sigma(\mu)$ is strictly decreasing in $\mu$. We also need the function

$$
v_{-}(\mu ; x)=v(\mu ;-x)
$$

which was used in Weinberger (1982) as well.

For the positive number $\alpha$ and $\ell$ satisfying $c^{*}(\ell)=c^{*}(\infty)-\epsilon$, we recall the function which was defined in Li et al. (2015) as follows. Let

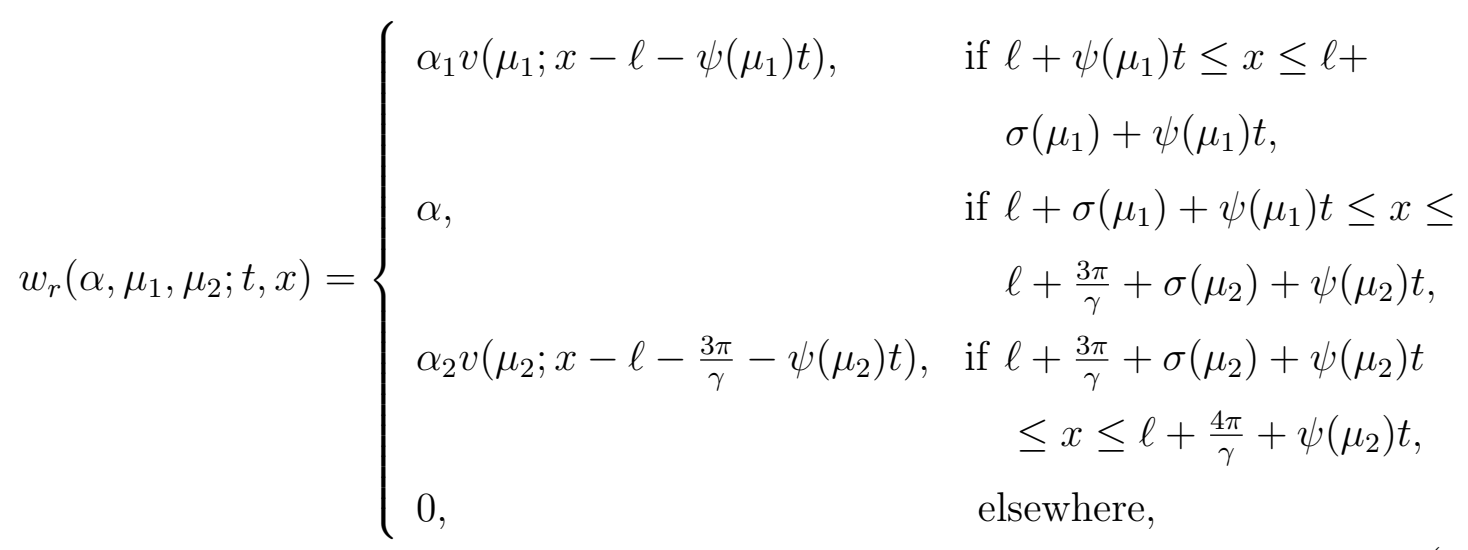

where $\alpha_{1}=\frac{\alpha}{v\left(\mu_{1} ; \sigma\left(\mu_{1}\right)\right)}, \alpha_{2}=\frac{\alpha}{v\left(\mu_{2} ; \sigma\left(\mu_{2}\right)\right)}$ and $\psi(\mu)$ is defined by formula (8). For $x$ in the interval

$$
\left[\ell+\sigma\left(\mu_{1}\right)+\psi\left(\mu_{1}\right) t, \ell+\frac{3 \pi}{\gamma}+\sigma\left(\mu_{2}\right)+\psi\left(\mu_{2}\right) t\right],
$$

we have $w_{r}\left(\alpha, \mu_{1}, \mu_{2} ; t, x\right)=\alpha$, with the end points shifting rightward at speeds $\psi\left(\mu_{1}\right)$ and $\psi\left(\mu_{2}\right)$ as $t \rightarrow \infty$. 
We define two similar functions. The first one is that

$$
w\left(\alpha, \mu_{1}, \mu_{2} ; t, x\right)=\left\{\begin{array}{lc}
\alpha_{1} v_{-}\left(\mu_{1} ; x-\ell+\psi\left(\mu_{1}\right) t\right), & \text { if } \ell-\psi\left(\mu_{1}\right) t-\frac{\pi}{\gamma} \leq x \leq \ell- \\
\alpha, & \sigma\left(\mu_{1}\right)-\psi\left(\mu_{1}\right) t, \\
& \text { if } \ell-\sigma\left(\mu_{1}\right)-\psi\left(\mu_{1}\right) t \leq x \leq \\
\alpha_{2} v\left(\mu_{2} ; x-\ell-\frac{3 \pi}{\gamma}-\psi\left(\mu_{2}\right) t\right), & \\
& \ell+\frac{3 \pi}{\gamma}+\sigma\left(\mu_{2}\right)+\psi\left(\mu_{2}\right) t \\
0, & \leq x \leq \ell+\frac{3 \pi}{\gamma}+\sigma\left(\mu_{2}\right)+\psi\left(\mu_{2}\right) t \\
0 & \text { elsewhere },
\end{array}\right.
$$

where $\alpha_{1}=\frac{\alpha}{v_{-}\left(\mu_{1} ;-\sigma\left(\mu_{1}\right)\right)}$ and $\alpha_{2}=\frac{\alpha}{v\left(\mu_{2} ; \sigma\left(\mu_{2}\right)\right)}$. For $x$ in the interval

$$
\left[\ell-\sigma\left(\mu_{1}\right)-\psi\left(\mu_{1}\right) t, \ell+\frac{3 \pi}{\gamma}+\sigma\left(\mu_{2}\right)+\psi\left(\mu_{2}\right) t\right],
$$

we have $w\left(\alpha, \mu_{1}, \mu_{2} ; t, x\right)=\alpha$, with left end point shifting leftward at speeds $\psi\left(\mu_{1}\right)$ and right end point shifting rightward at speeds $\psi\left(\mu_{2}\right)$ as $t \rightarrow \infty$.

For the second function,

$$
w_{l}\left(\alpha, \mu_{1}, \mu_{2} ; t, x\right)=\left\{\begin{array}{lr}
\alpha_{1} v_{-}\left(\mu_{1} ; x-\ell+\psi\left(\mu_{1}\right) t\right), & \text { if } \ell-\psi\left(\mu_{1}\right) t-\frac{\pi}{\gamma} \leq x \leq \ell- \\
\alpha, & \sigma\left(\mu_{1}\right)-\psi\left(\mu_{1}\right) t, \\
& \text { if } \ell-\sigma\left(\mu_{1}\right)-\psi\left(\mu_{1}\right) t \leq x \leq \\
& \ell+\frac{3 \pi}{\gamma}-\sigma\left(\mu_{2}\right)-\psi\left(\mu_{2}\right) t \\
\alpha_{2} v_{-}\left(\mu_{2} ; x-\ell-\frac{3 \pi}{\gamma}+\psi\left(\mu_{2}\right) t\right), & \text { if } \ell+\frac{3 \pi}{\gamma}-\sigma\left(\mu_{2}\right)-\psi\left(\mu_{2}\right) t \\
& \leq x \leq \ell+\frac{3 \pi}{\gamma}-\psi\left(\mu_{2}\right) t \\
0, & \text { elsewhere },
\end{array}\right.
$$

where $\alpha_{1}=\frac{\alpha}{v_{-}\left(\mu_{1} ;-\sigma\left(\mu_{1}\right)\right)}$ and $\alpha_{2}=\frac{\alpha}{v_{-}\left(\mu_{2} ;-\sigma\left(\mu_{2}\right)\right)}$. For $x$ in the interval

$$
\left[\ell-\sigma\left(\mu_{1}\right)-\psi\left(\mu_{1}\right) t, \ell+\frac{3 \pi}{\gamma}-\sigma\left(\mu_{2}\right)-\psi\left(\mu_{2}\right) t\right],
$$

we have $w_{l}\left(\alpha, \mu_{1}, \mu_{2} ; t, x\right)=\alpha$, with both end points shifting leftward at speeds $\psi\left(\mu_{1}\right)$ and $\psi\left(\mu_{2}\right)$ as $t \rightarrow \infty$.

The following lemma was given in Li et al. (2015) (Lemma 2.2) for $-\infty<$ $r(-\infty)<0$, but is valid for the case of $0<r(-\infty)<r(\infty)$ as well. 
Lemma II.6 Assume that Hypothesis II.1 is satisfied and $0 \leq c<c^{*}(\infty)$. Assume also that $u(t, x)$ is a solution of model (3) with $0<u(0, x) \leq r(\infty)$ for all $x$. For any positive $\epsilon$, there exists $\ell$ satisfying $c^{*}(\ell)=c^{*}(\infty)-\epsilon$. Let $\psi\left(\mu_{1}\right)=c+\epsilon$ and $\psi\left(\mu_{2}\right)=c^{*}(\infty)-\epsilon$. Then there exist $a>0$ and $\gamma>0$ sufficiently small, such that the functions $\tilde{u}_{1}(t, x)=a v\left(\mu_{1} ; x-\ell-\psi\left(\mu_{1}\right) t\right)$ and $\tilde{u}_{2}(t, x)=a v\left(\mu_{2} ; x-\ell-\psi\left(\mu_{2}\right) t\right)$ with $v$ defined by (12) are continuous weak lower solutions of model (3). Furthermore if $u(0, x) \geq a v\left(\mu_{i}, x-\ell\right)$ for $i=1$ and 2 , then $u(t, x) \geq a v\left(\mu_{1}, x-\ell-\psi\left(\mu_{1}\right) t\right)$ and $u(t, x) \geq \operatorname{av}\left(\mu_{2}, x-\ell-\psi\left(\mu_{2}\right) t\right)$ for all $t>0$.

The proof of this lemma is similar to the proof for Lemma 2.2 in Li et al. (2015), so we omit the details.

Similar to Lemma II.6, the following lemma is about the lower solution of model (3) when $c \geq c^{*}(\infty)$.

Lemma II.7 Assume that Hypothesis II.1 is satisfied and $c \geq c^{*}(\infty)$. Assume also that $u(t, x)$ is a solution of model (3) with $0<u(0, x) \leq r(\infty)$ for all $x$. For any positive $\epsilon$, there exists $\ell$ satisfying $c^{*}(\ell)=c^{*}(\infty)-\epsilon$. Let $\psi\left(\mu_{1}\right)=\psi\left(\mu_{2}\right)=c^{*}(-\infty)-\epsilon$. Then there exist $a>0$ and $\gamma>0$ sufficiently small, such that the functions $\tilde{u}_{1}(t, x)=$ $a v_{-}\left(\mu_{1} ; x-\ell+\psi\left(\mu_{1}\right) t\right)$ and $\tilde{u}_{2}(t, x)=a v\left(\mu_{2} ; x-\ell-\psi\left(\mu_{2}\right) t\right)$ with $v$ defined by (12) and $v_{-}$defined by (13) are continuous weak lower solutions of model (3). Furthermore if $u(0, x) \geq a v_{-}\left(\mu_{1}, x-\ell\right)$ and $u(0, x) \geq a v\left(\mu_{2}, x-\ell\right)$, then $u(t, x) \geq a v_{-}\left(\mu_{1}, x-\ell+\right.$ $\left.\psi\left(\mu_{1}\right) t\right)$ and $u(t, x) \geq \operatorname{av}\left(\mu_{2}, x-\ell-\psi\left(\mu_{2}\right) t\right)$ for all $t>0$.

Proof. When $\mu=\mu_{1}$, let $z=\psi(\mu)=2 d \mu_{1}$. By using the definition of $v_{-}$and integration by parts, we have that

$$
\begin{aligned}
& \int_{0}^{T_{1}} \int_{-\infty}^{+\infty} v_{-}(\mu ; x-\ell+z s)\left(d \eta_{x x}+\eta_{t}\right)(s, x) d x d s \\
= & \int_{0}^{T_{1}} \int_{\ell-\frac{\pi}{\gamma}-z s}^{\ell-z s} v_{-}(\mu ; x-\ell+z s)\left(d \eta_{x x}+\eta_{t}\right)(s, x) d x d s \\
= & \int_{0}^{T_{1}} \int_{\ell-\frac{\pi}{\gamma}-z s}^{\ell-z s}\left[d\left(v_{-}\right)_{x x}(\mu ; x-\ell+z s)-\left(v_{-}\right)_{s}(\mu ; x-\ell+z s)\right] \eta(s, x) d x d s \\
& +\left.\int_{\ell-\frac{\pi}{\gamma}-z s}^{\ell-z s} v_{-}(\mu ; x-\ell+z s) \eta(s, x) d x\right|_{0} ^{T_{1}} \\
& +\gamma d \int_{0}^{T_{1}}\left[e^{-\mu \frac{\pi}{\gamma}} \eta\left(s, \ell-z s+\frac{\pi}{\gamma}\right)+\eta(s, \ell-z s)\right] d s .
\end{aligned}
$$


By direct calculation, we have that for $x \neq \ell-z t$, and $x \neq \ell-z t-\pi / \gamma$,

$$
d v_{x x}(\mu ; x-\ell+z t)-v_{t}(\mu ; x-\ell+z t)=-d\left(\mu^{2}+\gamma^{2}\right) v_{-}(\mu ;(x-\ell+z t)) .
$$

Consider the definition of weak lower solutions and also the results of (17) and (18). Let $f(t, x, u)=u(r(x-c t)-u)$, then for sufficiently small $a$ and $\gamma, \tilde{u}_{1}(t, x)$ satisfies

$$
\begin{aligned}
& \int_{0}^{T_{1}} \int_{-\infty}^{+\infty}\left[\tilde{u}_{1}(s, x)\left(d \eta_{x x}+\eta_{t}\right)(s, x)+\eta(s, x) f\left(s, x, \tilde{u}_{1}(s, x)\right)\right] d x d s \\
& -\left.\int_{-\infty}^{\infty} \tilde{u}_{1}(s, x) \eta(s, x) d x\right|_{t=0} ^{t=T_{1}} \\
= & a \int_{0}^{T_{1}} \int_{\ell-z s-\pi / \gamma}^{\ell-z s}\left[r(x-c s)-d \mu^{2}-d \gamma^{2}-a v_{-}(\mu ; x-\ell+z s)\right] \\
& \cdot v_{-}(\mu ; x-\ell+z s) \eta(s, x) d x d s+a \gamma d \int_{0}^{T_{1}}\left[e^{-\mu \frac{\pi}{\gamma}} \eta\left(s, \ell-z s+\frac{\pi}{\gamma}\right)+\eta(s, \ell-z s)\right] d s \\
\geq & a \int_{0}^{T_{1}} \int_{\ell-z s-\pi / \gamma}^{\ell-z s}\left[r(-\infty)-d \mu^{2}-d \gamma^{2}-a v_{-}(\mu ; x-\ell+z s)\right] \\
& \cdot v_{-}(\mu ; x-\ell+z s) \eta(s, x) d x d s+a \gamma d \int_{0}^{T_{1}}\left[e^{-\mu \frac{\pi}{\gamma}} \eta\left(s, \ell-z s+\frac{\pi}{\gamma}\right)+\eta(s, \ell-z s)\right] d s \\
= & a \int_{0}^{T_{1}} \int_{\ell-z s-\pi / \gamma}^{\ell-z s}\left[\frac{\left(2 * c^{*}(-\infty)-\epsilon\right) \epsilon}{4 d}-d \gamma^{2}-a v_{-}(\mu ; x-\ell+z s)\right] \\
& \cdot v_{-}(\mu ; x-\ell+z s) \eta(s, x) d x d s+a \gamma d \int_{0}^{T_{1}}\left[e^{-\mu \frac{\pi}{\gamma}} \eta\left(s, \ell-z s+\frac{\pi}{\gamma}\right)+\eta(s, \ell-z s)\right] d s \\
\geq & 0 .
\end{aligned}
$$

According to the definition of a weak lower solution, when $u(0, x) \geq a v_{-}\left(\mu_{1}, x-\ell\right)$ for $x \in[\ell, \ell+\pi / \gamma]$ then $u(t, x) \geq a v_{-}\left(\mu_{1}, x-\ell-\psi\left(\mu_{1}\right) t\right)$ for all $t>0$, i.e. $\tilde{u}_{1}(t, x)$ is a weak lower solution of model (3).

When $\mu=\mu_{2}$, the function $\tilde{u}_{2}(t, x)=a v\left(\mu_{2} ; x-\ell-\psi\left(\mu_{2}\right) t\right)$ satisfies equations (17) and (18). Since $r(x-c s)-d \mu_{2}^{2} \geq \frac{\left(2 * c^{*}(-\infty)-\epsilon\right) \epsilon}{4 d}$, we have that $\tilde{u}_{2}(t, x)$ is valid for the process of (19). Therefore, $\tilde{u}_{2}(t, x)$ is a weak lower solution of model (3).

The proof is complete.

Similarly, when $c^{*}(-\infty)>-c \geq 0$, we have the following lemma for the lower solution of model (3).

Lemma II.8 Assume that Hypothesis II.1 is satisfied and $c^{*}(-\infty)>-c \geq 0$. Assume also that $u(t, x)$ is a solution of model (3) with $0<u(0, x) \leq r(\infty)$ for all $x$. For any positive $\epsilon$, there exists $\ell$ satisfying $c^{*}(\ell)=c^{*}(\infty)-\epsilon$. Let $\psi\left(\mu_{1}\right)=-c-\epsilon$, 
$\psi\left(\mu_{2}\right)=c^{*}(\infty)-2 \epsilon, \psi\left(\mu_{3}\right)=c^{*}(-\infty)-\epsilon$, and $\psi\left(\mu_{4}\right)=-c+\epsilon$. Then there exist $a>0$ and $\gamma>0$ sufficiently small, such that if $u(0, x) \geq a v_{-}\left(\mu_{i}, x-\ell\right)$ for $i=1,3,4$ and $u(0, x) \geq a v\left(\mu_{2}, x-\ell\right)$, then $u(t, x) \geq a v_{-}\left(\mu_{1}, x-\ell+\psi\left(\mu_{1}\right) t\right)$, $u(t, x) \geq a v\left(\mu_{2}, x-\ell-\psi\left(\mu_{2}\right) t\right), u(t, x) \geq a v_{-}\left(\mu_{3}, x-\ell+\psi\left(\mu_{3}\right) t\right)$ and $u(t, x) \geq$ $a v_{-}\left(\mu_{4}, x-\ell+\psi\left(\mu_{4}\right) t\right)$ for all $t>0$.

The proof of this lemma is similar to the previous Lemma II.7. When $\mu=\mu_{1}$, we have that

$$
\begin{aligned}
r(x-c s)-d \mu_{1}^{2} & \geq r\left(\ell-\frac{\pi}{\gamma}-\psi\left(\mu_{1}\right) s-c s\right)-d \mu_{1}^{2} \\
& =r\left(\ell-\frac{\pi}{\gamma}+(c+\epsilon) s-c s\right)-d \mu_{1}^{2} \\
& \geq r(\ell)-d \mu_{1}^{2} \\
& \geq \frac{\left(c^{*}(\infty)-\epsilon\right)^{2}}{4 d}-\frac{(c+\epsilon)^{2}}{4 d}>0
\end{aligned}
$$

and when $\mu_{2}$, we have that

$$
\begin{aligned}
r(x-c s)-d \mu_{2}^{2} & \geq r\left(\ell+\psi\left(\mu_{2}\right) s-c s\right)-d \mu_{2}^{2} \\
& =r\left(\ell+\left(c^{*}(\infty)-\epsilon\right) s-c s\right)-d \mu_{2}^{2} \\
& \geq r(\ell)-d \mu_{2}^{2} \\
& \geq \frac{\left(c^{*}(\infty)-\epsilon\right)^{2}}{4 d}-\frac{\left(c^{*}(\infty)-2 \epsilon\right)^{2}}{4 d}>0 .
\end{aligned}
$$

For $\mu=\mu_{3}$ and $\mu=\mu_{4}$, we have that $r(x-c s)>r(-\infty)$. We omit the details of the proof here.

Additionally, we have lemmas for $c^{*}(-\infty) \leq-c<c^{*}(\infty)$ and $-c \geq c^{*}(\infty)$. We omit the proof here.

Lemma II.9 Assume that Hypothesis II.1 is satisfied and $c^{*}(-\infty) \leq-c<c^{*}(\infty)$. Assume also that $u(t, x)$ is a solution of model (3) with $0<u(0, x) \leq r(\infty)$ for all $x$. For any positive $\epsilon$, there exists $\ell$ satisfying $c^{*}(\ell)=c^{*}(\infty)-\epsilon$. Let $\psi\left(\mu_{1}\right)=-c-\epsilon$, and $\psi\left(\mu_{2}\right)=c^{*}(\infty)-\epsilon$. Then there exist $a>0$ and $\gamma>0$ sufficiently small, such that if $u(0, x) \geq a v_{-}(\mu, x-\ell)$ and $u(0, x) \geq a v(\mu, x-\ell)$, then $u(t, x) \geq a v_{-}\left(\mu_{1}, x-\ell+\psi\left(\mu_{1}\right) t\right)$ and $u(t, x) \geq a v\left(\mu_{2}, x-\ell-\psi\left(\mu_{2}\right) t\right)$ for all $t>0$. 
Lemma II.10 Assume that Hypothesis II.1 is satisfied and $-c \geq c^{*}(\infty)$. Assume also that $u(t, x)$ is a solution of model (3) with $0<u(0, x) \leq r(\infty)$ for all $x$. For any positive $\epsilon$, there exists $\ell$ satisfying $c^{*}(\ell)=c^{*}(\infty)-\epsilon$. Let $\psi\left(\mu_{1}\right)=\psi\left(\mu_{2}\right)=c^{*}(\infty)-\epsilon$. Then there exist $a>0$ and $\gamma>0$ sufficiently small, such that if $u(0, x) \geq a v_{-}(\mu, x-\ell)$ and $u(0, x) \geq a v(\mu, x-\ell)$, then $u(t, x) \geq a v_{-}\left(\mu_{1}, x-\ell+\psi\left(\mu_{1}\right) t\right)$ and $u(t, x) \geq$ av $\left(\mu_{2}, x-\ell-\psi\left(\mu_{2}\right) t\right)$ for all $t>0$.

The following lemma shows that functions $w_{l}\left(\alpha, \mu_{1}, \mu_{2} ; t, x\right), w_{r}\left(\alpha, \mu_{1}, \mu_{2} ; t, x\right)$ and $w\left(\alpha, \mu_{1}, \mu_{2} ; t, x\right)$ can translate to the lower solutions of system (3) under appropriate conditions.

Lemma II.11 Assume that Hypotheses II.1 is satisfied and $u(t, x)$ is a solution of model (3) with $0<u(0, x) \leq r(\infty)$ for all $x$. Then for any small positive number $\epsilon$, there exist positive numbers $\alpha, \gamma, \mu_{1}, \mu_{2}$, and a positive number $t_{0}$. Let $\ell$ satisfy $c^{*}(\ell)=c^{*}(\infty)-\epsilon$. Then for $t>t_{0}$

(a) If $c>c^{*}(\infty), u(t, x) \geq w\left(\alpha, \mu_{1}, \mu_{2} ; t-t_{0}, x\right)$ with $\psi\left(\mu_{1}\right)=\psi\left(\mu_{2}\right)=c^{*}(-\infty)-\epsilon$;

(b) If $0<c<c^{*}(\infty), u(t, x) \geq w_{r}\left(\alpha, \mu_{1}, \mu_{2} ; t-t_{0}, x\right)$ with $\psi\left(\mu_{1}\right)=c+\epsilon$ and $\psi\left(\mu_{2}\right)=c^{*}(\infty)-\epsilon$; and, $u(t, x) \geq w\left(\alpha, \mu_{1}^{\prime}, \mu_{2}^{\prime} ; t-t_{0}, x\right)$ with $\psi\left(\mu_{1}^{\prime}\right)=\psi\left(\mu_{2}^{\prime}\right)=$ $c^{*}(-\infty)-\epsilon$;

(c) If $c^{*}(-\infty)>-c \geq 0, u(t, x) \geq w\left(\alpha, \mu_{1}, \mu_{2} ; t-t_{0}, x\right)$ with $\psi\left(\mu_{1}\right)=-c-\epsilon$ and $\psi\left(\mu_{2}\right)=c^{*}(\infty)-\epsilon$; and, $u(t, x) \geq w_{l}\left(\alpha, \mu_{1}^{\prime}, \mu_{2}^{\prime} ; t-t_{0}, x\right)$ with $\psi\left(\mu_{1}^{\prime}\right)=c^{*}(-\infty)-\epsilon$ and $\psi\left(\mu_{2}^{\prime}\right)=-c+\epsilon$;

(d) If $c^{*}(-\infty) \leq-c<c^{*}(\infty), u(t, x) \geq w\left(\alpha, \mu_{1}, \mu_{2} ; t-t_{0}, x\right)$ with $\psi\left(\mu_{1}\right)=-c-\epsilon$ and $\psi\left(\mu_{2}\right)=c^{*}(\infty)-\epsilon$;

(e) If $-c \geq c^{*}(\infty), u(t, x) \geq w\left(\alpha, \mu_{1}, \mu_{2} ; t-t_{0}, x\right)$ with $\psi\left(\mu_{1}\right)=\psi\left(\mu_{2}\right)=c^{*}(\infty)-\epsilon$.

Proof. Now we are going to prove statement $(\mathrm{d})$. Since $c^{*}(-\infty) \leq-c<c^{*}(\infty)$, for any $\epsilon$ with $0<\epsilon<\min \left\{1, \frac{c^{*}(\infty)+c}{3}\right\}$, let $\ell$ satisfy

$$
c^{*}(\ell)=c^{*}(\infty)-\epsilon .
$$


Assume that $\psi\left(\mu_{1}\right)=-c-\epsilon$ and $\psi\left(\mu_{2}\right)=c^{*}(\infty)-2 \epsilon$. By Lemma II.9, $u(t, x) \geq$ $a v_{-}\left(\mu_{1}, x-\ell+\psi\left(\mu_{1}\right) t\right)$ and $u(t, x) \geq a v\left(\mu_{2}, x-\ell-\psi\left(\mu_{2}\right) t\right)$ for all $t>0$.

Since $u(0, x) \geq 0$ and $u(0, x) \not \equiv 0, u(t, x)>0$ for any $t>0$. Choose $0<t_{0}<$ $\frac{\sigma\left(\mu_{1}\right)}{\psi\left(\mu_{1}\right)}$ and $\alpha$ and $\gamma$ are sufficiently small such that $u\left(t_{0}, x\right) \geq \alpha$ for $x \in[\ell-\pi / \gamma, \ell+4 \pi / \gamma]$. Let $\alpha_{1}=\frac{\alpha}{v_{-}\left(\mu_{1} ;-\sigma\left(\mu_{1}\right)\right)}$ and $\alpha_{2}=\frac{\alpha}{v\left(\mu_{2} ; \sigma\left(\mu_{2}\right)\right)}$. From formula (15), assuming $0 \leq s \leq 3 \pi / \gamma$, it is easy to see that

$$
\begin{aligned}
& w(0, x) \geq \alpha_{1} v_{-}\left(\mu_{1} ; x-\ell-s\right) \\
& w(0, x) \geq \alpha_{2} v\left(\mu_{2} ; x-\ell-3 \pi / \gamma+s\right) .
\end{aligned}
$$

Since $u\left(t_{0}, x\right) \geq \alpha$ for $x \in[\ell-\pi / \gamma, \ell+4 \pi / \gamma]$, from Lemma II.9 we have that for $t \geq t_{0}$ and $0 \leq s \leq 3 \pi / \gamma$

$$
\begin{gathered}
u(t, x) \geq \alpha_{1} v_{-}\left(\mu_{1} ; x-\ell+\psi\left(\mu_{1}\right)\left(t-t_{0}\right)-s\right), \\
u(t, x) \geq \alpha_{2} v\left(\mu_{2} ; x-\ell-3 \pi / \gamma-\psi\left(\mu_{2}\right)\left(t-t_{0}\right)+s\right) .
\end{gathered}
$$

Inequality (20) implies that for $t \geq t_{0}$,

$$
u(t, x) \geq \begin{cases}\alpha_{1} v\left(\mu_{1} ; x-\ell+\psi\left(\mu_{1}\right)\left(t-t_{0}\right)\right), & \text { if } \ell-\psi\left(\mu_{1}\right)\left(t-t_{0}\right)-\pi / \gamma \leq x \leq \\ & \ell-\sigma\left(\mu_{1}\right)-\psi\left(\mu_{1}\right)\left(t-t_{0}\right), \\ \alpha, & \text { if } \ell-\sigma\left(\mu_{1}\right)-\psi\left(\mu_{1}\right)\left(t-t_{0}\right) \leq x \leq \\ & \ell+\frac{3 \pi}{\gamma}-\sigma\left(\mu_{1}\right)-\psi\left(\mu_{1}\right)\left(t-t_{0}\right), \\ 0, & \text { elsewhere. }\end{cases}
$$

On the other hand, (21) indicates that for $t \geq t_{0}$,

$$
u(t, x) \geq\left\{\begin{array}{lc}
\alpha & \text { if } \ell+\sigma\left(\mu_{2}\right)+\psi\left(\mu_{2}\right)\left(t-t_{0}\right) \leq x \\
& \leq \ell+\frac{3 \pi}{\gamma}+\sigma\left(\mu_{2}\right)+\psi\left(\mu_{2}\right)\left(t-t_{0}\right), \\
\alpha_{2} v\left(\mu_{2} ; x-\ell-\frac{3 \pi}{\gamma}-\psi\left(\mu_{2}\right)\left(t-t_{0}\right)\right), & \text { if } \ell+\frac{3 \pi}{\gamma}+\sigma\left(\mu_{2}\right)+\psi\left(\mu_{2}\right)\left(t-t_{0}\right) \\
& \leq x \leq \ell+\frac{4 \pi}{\gamma}+\psi\left(\mu_{2}\right)\left(t-t_{0}\right), \\
0, & \text { elsewhere. }
\end{array}\right.
$$


Let $h=\frac{3 \pi / \gamma-\sigma\left(\mu_{1}\right)-\sigma\left(\mu_{2}\right)}{\psi\left(\mu_{2}\right)+\psi\left(\mu_{1}\right)}>0$, then we have that $\ell+3 \pi / \gamma-\sigma\left(\mu_{1}\right)-\psi\left(\mu_{1}\right)\left(t-t_{0}\right) \geq$ $\ell+\sigma\left(\mu_{2}\right)+\psi\left(\mu_{2}\right)\left(t-t_{0}\right)$ when $t_{0} \leq t \leq t_{0}+h$. In this case, combining (22) and (23) together, we have that

$$
u(t, x) \geq w\left(\alpha, \mu_{1}, \mu_{2} ; t-t_{0}, x\right)
$$

Now we are going to prove that (24) is valid for all $t \geq t_{0}$. Assume that (24) is true for $t_{0} \leq t \leq t_{0}+n h$ for some positive integers $n$. Then

$$
\begin{aligned}
& w(n h, x) \geq \alpha_{1} v_{-}\left(\mu_{1} ; x-\ell+n h \psi\left(\mu_{1}\right)-s\right), \\
& w(n h, x) \geq \alpha_{2} v\left(\mu_{2} ; x-\ell-3 \pi / \gamma-n h \psi\left(\mu_{2}\right)+s\right),
\end{aligned}
$$

where $0 \leq s \leq \frac{3 \pi}{\gamma}+\left(\psi\left(\mu_{1}\right)+\psi\left(\mu_{2}\right)\right) n h$. Therefore,

$$
\begin{gathered}
u(t, x) \geq \alpha_{1} v_{-}\left(\mu_{1} ; x-\ell-n h \psi\left(\mu_{1}\right)+\psi\left(\mu_{1}\right)\left(t-\left(t_{0}+n h\right)\right)-s\right), \\
u(t, x) \geq \alpha_{2} v\left(\mu_{2} ; x-\ell-3 \pi / \gamma-n h \psi\left(\mu_{2}\right)-\psi\left(\mu_{2}\right)\left(t-\left(t_{0}+n h\right)\right)+s\right) .
\end{gathered}
$$

Following the same method to prove that (24) is valid for $t_{0} \leq t \leq t_{0}+n h$, the interval for $u(t, x) \geq \alpha$ from equations (25) and (26) overlap when

$$
t_{0}+n h \leq t \leq t_{0}+2 n h+\frac{\frac{3 \pi}{\gamma}+\sigma\left(\mu_{1}\right)-\sigma\left(\mu_{2}\right)}{\psi\left(\mu_{1}\right)+\psi\left(\mu_{2}\right)}=t_{0}+(n+1) h .
$$

We therefore have that (24) holds if $t_{0}+n h \leq t \leq t_{0}+(n+1) h$. By induction, (24) holds for all $t \geq t_{0}$.

This proves the statement (d). By using a similar method and Lemmas II.7 through Lemma II.10, we can prove the rest of the statements in this lemma.

$5 \quad$ Spreading Speed

We provide a theorem to show that when $c$ is large, the species persists in space and spreads to both right and left at the asymptotic spreading speed $c^{*}(-\infty)$.

Theorem II.1 Assume that Hypotheses II.1 is satisfied and $c \geq c^{*}(\infty)$. Assume also $u(t, x)$ is a solution of model (3) with $0<u(0, x) \leq r(\infty)$ for all $x$. 
(a) Let $\mu^{+}=\mu^{*}(\infty)=\sqrt{\frac{r(\infty)}{d}}, \mu^{-}=\mu^{*}(-\infty)=\sqrt{\frac{r(-\infty)}{d}}$ and $\underline{c}=\frac{\mu^{+} c^{*}(\infty)-\mu^{-} c^{*}(-\infty)}{\mu^{+}-\mu^{-}}$. If $u(0, x)$ is zero for all sufficiently large $x, 0 \leq u(0, x) \leq r(\infty)$, and $c>\underline{c}$, then for any positive $\epsilon$,

$$
\lim _{t \rightarrow+\infty}\left[\sup _{x \geq t\left(c^{*}(-\infty)+\epsilon\right)} u(t, x)\right]=0
$$

(b) If $u(0, x)$ is zero for all sufficiently negative $x$, and $0 \leq u(0, x) \leq r(\infty)$, then for any positive $\epsilon$

$$
\lim _{t \rightarrow+\infty}\left[\sup _{x \leq-t\left(c^{*}(-\infty)+\epsilon\right)} u(t, x)\right]=0 .
$$

(c) If $u(0, x)>0$ on a closed interval and $0 \leq u(0, x) \leq r(\infty)$, then for every $\epsilon>0$

$$
\lim _{t \rightarrow+\infty}\left[\inf _{-t\left(c^{*}(-\infty)-\epsilon\right) \leq x \leq t\left(c^{*}(-\infty)-\epsilon\right)} u(t, x)\right]=r(-\infty) .
$$

Remark II.1 Biologically, this theorem implies that, if the species spreading speed in the high quality environment is not sufficient to keep pace with the rate of climate change, then the species can effectively only exist in the low quality environment. Since the species is spreading only in the low quality environment, its leftward and rightward spreading speeds are given by $c^{*}(-\infty)$. This theorem does not include the case of $c^{*}(\infty)<c \leq \underline{c}$. This case is discussed in Figure 1a, where our numerical simulation suggests that both leftward and rightward spreading speeds are still $c^{*}(-\infty)$.

Proof. We first prove statement (a). From statement (a) of Lemma II.4, we have that for any small positive number $\epsilon$, there exist $A_{1}>0$, such that

$$
u(t, x) \leq u_{1}(t, x)=A_{1} e^{-\mu_{1}\left(x-\left(c^{*}(\infty)+\epsilon\right) t\right)},
$$

where $\mu_{1}$ is the smallest solution of $\phi(\infty ; \mu)=c^{*}(\infty)+\epsilon$. Additionally, from the formula (11), for the given $\epsilon$, there exists $\delta>0$ and $0<\mu_{2}<\mu^{*}(-\infty)$, such that $\mu_{2}\left(c^{*}(-\infty)+\epsilon / 2\right)=d \mu_{2}^{2}+r(-\infty)+\delta$. We assume that

$$
u_{2}(t, x)=A_{2} e^{-\mu_{2}\left(x-\left(c^{*}(-\infty)+\epsilon\right) t\right)}
$$

and $S=\frac{\ln A_{1}-\ln A_{2}}{\mu_{1}-\mu_{2}}$. It follows that

$$
\mu^{+} \geq \mu_{1}=\mu^{+}+\frac{\epsilon-\sqrt{\epsilon^{2}+4 \epsilon \sqrt{d r(\infty)}}}{2 d}>\mu^{+}-\frac{\epsilon}{2 d},
$$




$$
\mu^{-} \geq \mu_{2}=\mu^{-}+\frac{\epsilon-\sqrt{\epsilon^{2}+4 \epsilon \sqrt{d r(-\infty)}-4 d \delta}}{2 d}>\mu^{-}-\frac{\epsilon}{2 d} .
$$

By direct calculation,

$$
\begin{gathered}
\frac{\mu_{1} c^{*}(\infty)-\mu_{2} c^{*}(-\infty)}{\mu_{1}-\mu_{2}} \\
=\frac{\mu^{+} c^{*}(\infty)-\mu^{-} c^{*}(-\infty)+\left(\mu^{+}-\mu^{-}\right) \epsilon+\left(\sqrt{\epsilon^{2}+4 \epsilon \sqrt{d r^{-}}-4 d \delta} \mu^{-}-\sqrt{\epsilon^{2}+4 \epsilon \sqrt{d r^{+}}} \mu^{+}\right)}{\mu^{+}-\mu^{-}+\frac{1}{2 d}\left(\sqrt{\epsilon^{2}+4 \epsilon \sqrt{d r^{-}}-4 d \delta}-\sqrt{\epsilon^{2}+4 \epsilon \sqrt{d r^{+}}}\right)} .
\end{gathered}
$$

This formula converges to $\underline{c}$ as $\epsilon \rightarrow 0$. Choose $\epsilon$ sufficiently small so that

$$
0<\frac{\mu_{1} c^{*}(\infty)-\mu_{2} c^{*}(-\infty)}{\mu_{1}-\mu_{2}} \leq \underline{c}+\epsilon .
$$

When $c>\underline{c}+3 \epsilon$ and $x>(c-\epsilon) t+S$, we have that

$$
\begin{aligned}
\frac{u_{2}(t, x)}{u_{1}(t, x)} & =\frac{A_{2}}{A_{1}} e^{\mu_{1}\left(x-\left(c^{*}(\infty)+\epsilon\right) t\right)-\mu_{2}\left(x-\left(c^{*}(-\infty)+\epsilon\right) t\right)} \\
& =\frac{A_{2}}{A_{1}} e^{\left(\mu_{1}-\mu_{2}\right)\left[x-\left(\frac{\mu_{1} c^{*}(\infty)-\mu_{2} c^{*}(-\infty)}{\mu_{1}-\mu_{2}}+\epsilon\right) t\right]} \\
& \geq \frac{A_{2}}{A_{1}} e^{\left(\mu_{1}-\mu_{2}\right)[x-(\underline{c}+2 \epsilon) t]} \\
& \geq \frac{A_{2}}{A_{1}} e^{\left(\mu_{1}-\mu_{2}\right)(S+(c-\epsilon) t-(c-\epsilon) t)} \\
& \geq \frac{A_{2}}{A_{1}} e^{\left(\mu_{1}-\mu_{2}\right) S}=1
\end{aligned}
$$

It follows that, when $x>(c-\epsilon) t+S, u(t, x) \leq u_{1}(t, x) \leq u_{2}(t, x)$.

On the other hand, we consider the case of $x \leq x_{0}+c t-b$, where $x_{0}$ satisfies that for any $x<x_{0}, r(x)<r(-\infty)+\delta$. Assume $u(0, x) \leq u_{2}(0, x)=A_{2} e^{-\mu_{2} x}$ and $b$ is introduced in Lemma II.5 with $\mu=\mu_{2}$. The sequence $u^{(n)}(t, x)$ is defined in the formula (7) with $u^{(0)}(t, x)=0$. Obviously, $u^{(0)}(t, x)=0$ is a lower solution of system (3) and $u(t, x)=\lim _{n \rightarrow \infty} u^{(n)}(t, x)$. We have that

$$
\begin{aligned}
u^{(1)}(t, x) & =Q\left[u^{(0)}\right](t, x)=\int_{-\infty}^{+\infty} k(t, x-y) u_{0}(y) d y \\
& =A_{2} e^{-\rho t-\mu_{2} x+d \mu_{2}^{2} t} \\
& =u_{2}(t, x) e^{-\rho t-(r(-\infty)+\delta) t} \leq u_{2}(t, x) .
\end{aligned}
$$


We compute that

$$
\begin{aligned}
& Q\left[u_{2}\right](t, x)=\int_{-\infty}^{+\infty} k(t, x-y) u_{0}(y) d y+ \\
& \int_{0}^{t} \int_{-\infty}^{+\infty} k(t-\tau, x-y) u_{2}(\tau, y)\left[\rho+r(y-c \tau)-u_{2}(\tau, y)\right] d y d \tau \\
& \leq \int_{-\infty}^{+\infty} k(t, y) A_{2} e^{-\mu_{2}(x-y)} d y+ \\
& \int_{0}^{t} \int_{-\infty}^{+\infty} k(\tau, y) A_{2} e^{-\mu_{2}\left(x-y-\left(c^{*}(-\infty)+\epsilon\right)(t-\tau)\right.}[\rho+r(x-y-c(t-\tau))] d y d \tau \\
& \leq \int_{-\infty}^{+\infty} k(t, y) A_{2} e^{-\mu_{2}(x-y)} d y+\left(1+S_{0} \epsilon_{1}\right) \text {. } \\
& \int_{0}^{t} \int_{c \tau-b}^{+\infty} k(\tau, y) A_{2} e^{-\mu_{2}\left(x-y-\left(c^{*}(-\infty)+\epsilon\right)(t-\tau)\right.}[\rho+r(x-y-c(t-\tau))] d y d \tau \\
& \leq \int_{-\infty}^{+\infty} k(t, y) A_{2} e^{-\mu_{2}(x-y)} d y+\left(1+S_{0} \epsilon_{1}\right)(\rho+r(-\infty)+\delta) \cdot \\
& \int_{0}^{t} \int_{c \tau-b}^{+\infty} k(\tau, y) A_{2} e^{-\mu_{2}\left(x-y-\left(c^{*}(-\infty)+\epsilon\right)(t-\tau)\right.} d y d \tau \\
& \leq \int_{-\infty}^{+\infty} k(t, y) A_{2} e^{-\mu_{2}(x-y)} d y+(\rho+r(-\infty)+\delta)\left(1+S_{0} \epsilon_{1}\right) . \\
& \int_{0}^{t} e^{\mu_{2}\left(c^{*}(-\infty)+\epsilon\right)(t-\tau)} \int_{-\infty}^{+\infty} k(\tau, y) A_{2} e^{-\mu_{2}(x-y)} d y d \tau \\
& \leq A_{2} e^{-\rho t-\mu_{2} x+d \mu_{2}^{2} t}+(\rho+r(-\infty)+\delta)\left(1+S_{0} \epsilon_{1}\right) . \\
& \int_{0}^{t} e^{\mu_{2}\left(c^{*}(-\infty)+\epsilon\right)(t-\tau)} A_{2} e^{-\rho \tau-\mu_{2} x+d \mu_{2}^{2} \tau} d \tau \\
& =A_{2} e^{-\rho t-\mu_{2} x+d \mu_{2}^{2} t}+\frac{\rho+r(-\infty)+\delta}{d \mu_{2}^{2}-\rho-\mu_{2}\left(c^{*}(-\infty)+\epsilon\right)}\left(1+S_{0} \epsilon_{1}\right) \text {. } \\
& A e^{-\mu_{2}\left(x-\left(c^{*}(-\infty)+\epsilon\right) t\right)}\left(e^{\left(d \mu_{2}^{2}-\rho-\mu_{2}\left(c^{*}(-\infty)+\epsilon\right)\right) t}-1\right) \\
& =u_{2}(t, x) \text {. }
\end{aligned}
$$

By induction, $u^{(n)}(t, x) \leq u_{2}(t, x)$. Since $u^{(n)}(t, x)$ converges to $u(t, x)$, so $u(t, x) \leq$ $u_{2}(t, x)$ for any $x \leq x_{0}+c t-b$.

When $t>T_{\epsilon}=\frac{\left(S+b-x_{0}\right)}{\epsilon}$, we have that $(c-\epsilon) t+S \leq x_{0}+c t-b$. Therefore, $u(t, x) \leq u_{2}(t, x)$ for any $x$ and $t$ sufficiently large.

Now we consider the statement (b). From the statement (b) in Lemma II.4, for any $\epsilon>0$, there exist positive numbers $B$ and $\mu_{1}$ such that

$$
0 \leq u(t, x) \leq B e^{\mu_{1}\left(x+\left(c^{*}(-\infty)+\frac{\epsilon}{2}\right) t\right)} .
$$


It follows that

$$
\begin{aligned}
0 \leq \lim _{t \rightarrow+\infty}\left[\sup _{x \leq-t\left(c^{*}(-\infty)+\epsilon\right)} u(t, x)\right] & \leq \lim _{t \rightarrow+\infty}\left[\sup _{x \leq-t\left(c^{*}(-\infty)+\epsilon\right)} B e^{\mu_{1}\left(x+\left(c^{*}(-\infty)+\frac{\epsilon}{2}\right) t\right)}\right] \\
& \leq \lim _{t \rightarrow+\infty}\left[B e^{\mu_{1}\left(-t\left(c^{*}(-\infty)+\epsilon\right)+\left(c^{*}(-\infty)+\frac{\epsilon}{2}\right) t\right)}\right] \\
& \leq \lim _{t \rightarrow+\infty}\left[B e^{-\mu_{1} \frac{\epsilon}{2} t}\right]=0 .
\end{aligned}
$$

Therefore,

$$
\lim _{t \rightarrow+\infty}\left[\sup _{x \leq-t\left(c^{*}(-\infty)+\epsilon\right)} u(t, x)\right]=0 .
$$

Finally, we prove the statement (c). By using the conclusion in Lemma II.2, for any $\epsilon>0$, there exists sufficiently large $T_{1}>0$ and $M>0$, such that, when $t>T_{1}$ and $x \leq-M+c t$

$$
u(t, x) \leq r(-\infty)+\epsilon
$$

From Lemma II.4, we have that if $u(0, x)$ is zero for all sufficiently large $x$, and $0 \leq u(0, x) \leq r(\infty)$, then for any positive $\epsilon$ there exist positive numbers $A$ and $\mu_{\epsilon}$ such that

$$
u(t, x) \leq A e^{-\mu_{\epsilon}\left(x-\left(c^{*}(\infty)+\frac{\epsilon}{2}\right) t\right)}
$$

When $x \geq\left(c^{*}(\infty)+\epsilon\right) t$,

$$
u(t, x) \leq A e^{-\mu_{\epsilon} \frac{\epsilon}{2} t}
$$

It follows that, for the given $\epsilon$, there exists a sufficiently large $T_{2}$, such that

$$
u(t, x)<r(-\infty)+\epsilon \text { for } t \geq T_{2} \text { and } x \geq\left(c^{*}(\infty)+\epsilon\right) t
$$

Since $c^{*}(\infty)<c$, there exists $T_{3}>\max \left\{T_{1}, T_{2}\right\}$ such that for $t>T_{3},-M+c t>$ $\left(c^{*}(\infty)+\epsilon\right) t$. Therefore, when $t>T_{3}$, we have that $u(t, x) \leq r(-\infty)+\epsilon$ for any $x$.

On the other hand, from Lemma II.11, for any small positive number $\epsilon$, there exist $\alpha, \gamma, \mu_{1}, \mu_{2}$, and a positive number $t_{0}$, such that when $t>t_{0}$,

$$
u(t, x) \geq w\left(\alpha, \mu_{1}, \mu_{2} ; t-t_{0}, x\right)
$$

where $\psi\left(\mu_{1}\right)=\psi\left(\mu_{2}\right)=c^{*}(-\infty)-\epsilon / 2$.

For the given $\epsilon>0$, there is $L>0$ such that

$$
\int_{-L}^{L} \frac{1}{\sqrt{\pi}} e^{-x^{2}} d x \geq 1-\epsilon
$$


which also satisfies that

$$
\int_{-L \sqrt{4 d s}}^{L \sqrt{4 d s}} \frac{1}{\sqrt{4 \pi s}} e^{-\frac{x^{2}}{4 d s}} d x=\int_{-L}^{L} \frac{1}{\sqrt{\pi}} e^{-\xi^{2}} d \xi
$$

for any $s>0 . u\left(t_{0}, x\right)$ is assumed to be the initial value for the model $(3)$. Then the solution $u(t, x)$ satisfies the integral equation for $t>t_{0}$ :

$$
\begin{aligned}
u(t, x)= & \int_{-\infty}^{+\infty} k\left(t-t_{0}, x-y\right) u\left(t_{0}, y\right) d y \\
& +\int_{t_{0}}^{t} \int_{-\infty}^{+\infty} k(t-\tau, x-y) u(\tau, y)[\rho+r(y-c \tau)-u(\tau, y)] d y d \tau .
\end{aligned}
$$

When $t>t_{0}$ and $x$ satisfies

$\ell-\sigma\left(\mu_{1}\right)-\psi\left(\mu_{1}\right)\left(t-t_{0}\right)+L \sqrt{4 d\left(t-t_{0}\right)} \leq x \leq \ell+3 \pi / \gamma+\sigma\left(\mu_{2}\right)+\psi\left(\mu_{2}\right)\left(t-t_{0}\right)-L \sqrt{4 d\left(t-t_{0}\right)}$,

and $y$ satisfies

$$
-L \sqrt{4 d\left(t-t_{0}\right)} \leq y \leq L \sqrt{4 d\left(t-t_{0}\right)}
$$

we have that

$$
\ell-\sigma\left(\mu_{1}\right)-\psi\left(\mu_{1}\right)\left(t-t_{0}\right) \leq x-y \leq \ell+3 \pi / \gamma+\sigma\left(\mu_{2}\right)+\psi\left(\mu_{2}\right)\left(t-t_{0}\right) .
$$

By direct calculation,

$$
\int_{-\infty}^{+\infty} k\left(t-t_{0}, x-y\right) u\left(t_{0}, y\right) d y \geq(1-\epsilon) \alpha e^{-\rho\left(t-t_{0}\right)}
$$

and

$$
\int_{-\infty}^{+\infty} k(t-\tau, x-y) u^{(0)}(\tau, y)\left[\rho+r(y-c \tau)-u^{(0)}(\tau, y)\right] d y \geq(1-\epsilon) \alpha[\rho+r(-\infty)-\epsilon-\alpha] e^{-\rho(t-\tau)}
$$

It follows from (27)-(32) that for $t \geq t_{0}$ and $x$ satisfying (28)

$$
u(t, x) \geq \tilde{u}^{(1)}(t)
$$

where

$$
\tilde{u}^{(1)}(t)=(1-\epsilon) \alpha e^{-\rho\left(t-t_{0}\right)}+\int_{t_{0}}^{t} e^{-\rho(t-\tau)}(1-\epsilon) \alpha[\rho+r(-\infty)-\epsilon-\alpha] d \tau
$$


It follows from this, (27), and induction that for $t \geq t_{0}$ and $x$ satisfying (28),

$$
u(t, x) \geq \tilde{u}^{(n)}(t)
$$

where $\tilde{u}^{(n)}(t)$ satisfies

$$
\begin{aligned}
\tilde{u}^{(n+1)}(t) & =(1-\epsilon) \alpha e^{-\rho\left(t-t_{0}\right)}+\int_{t_{0}}^{t} e^{-\rho(t-\tau)}(1-\epsilon) \tilde{u}^{(n)}(\tau)\left[\rho+r(-\infty)-\epsilon-\frac{1}{1-\epsilon} \tilde{u}^{(n)}(\tau)\right] d \tau \\
& =a_{n}+b_{n}(t) e^{-\rho\left(t-t_{0}\right)} .
\end{aligned}
$$

In this formula,

$$
\begin{gathered}
a_{n}=\frac{(1-\epsilon) a_{n-1}\left(\rho+r(-\infty)-\epsilon-a_{n-1}\right)}{\rho}, \\
a_{1}=\frac{(1-\epsilon) \alpha[\rho+r(-\infty)-\epsilon-\alpha]}{\rho},
\end{gathered}
$$

and $b_{n}(t)$ is the sum of polynomials, and products of polynomials and exponential functions in the form of $e^{-j \rho\left(t-t_{0}\right)}$ with $j$ a positive integer.

Since $\lim _{t \rightarrow \infty} \tilde{u}^{(n)}(t)=a_{n}$ and $\lim _{n \rightarrow \infty} a_{n}=r(-\infty)-\epsilon-\epsilon \rho /(1-\epsilon)$, there exists a positive integer $N$ sufficiently large and $t_{2}>t_{0}$ such that for $t>t_{2}$

$$
\tilde{u}^{(N)}(t) \geq r(-\infty)-\epsilon-\epsilon \rho /(1-\epsilon) .
$$

Choose $t_{1}>t_{2}$ sufficiently large such that when $t>t_{1}$,

$$
\begin{gathered}
\ell-\sigma\left(\mu_{1}\right)-\psi\left(\mu_{1}\right)\left(t-t_{0}\right)+L N \sqrt{4 d\left(t-t_{0}\right)} \\
\leq \ell+3 \pi / \gamma+\sigma\left(\mu_{2}\right)+\psi\left(\mu_{2}\right)\left(t-t_{0}\right)-L N \sqrt{4 d\left(t-t_{0}\right)},
\end{gathered}
$$

and also there exists $t_{3}>t_{1}$ such that when $t>t_{3}$

$$
\begin{gathered}
\ell+\sigma\left(\mu_{1}\right)-\psi\left(\mu_{1}\right)\left(t-t_{0}\right)+L \sqrt{4 d\left(t-t_{0}\right)} \leq-t\left(c^{*}(-\infty)-\epsilon\right) \leq t\left(c^{*}(-\infty)-\epsilon\right), \\
t\left(c^{*}(-\infty)-\epsilon\right) \leq \ell+3 \pi / \gamma+\sigma\left(\mu_{2}\right)+\psi\left(\mu_{2}\right)\left(t-t_{0}\right)-L N \sqrt{4 d\left(t-t_{0}\right)}
\end{gathered}
$$

Equations (33), (34) and (35) show that when $t>t_{3}$ and $-t\left(c^{*}(-\infty)-\epsilon\right) \leq x \leq$ $t\left(c^{*}(-\infty)-\epsilon\right)$

$$
\lim _{t \rightarrow \infty}\left[\inf _{-t\left(c^{*}(-\infty)-\epsilon\right) \leq x \leq t\left(c^{*}(-\infty)-\epsilon\right)} u(t, x)\right] \geq(1-\epsilon)(r(\infty)-\epsilon) .
$$


Since $\epsilon$ is arbitrary and $u(t, x) \leq r(\infty)$ for all $x$ and $t$, it follows that

$$
\lim _{t \rightarrow+\infty}\left[\inf _{-t\left(c^{*}(-\infty)-\epsilon\right) \leq x \leq t\left(c^{*}(-\infty)-\epsilon\right)} u(t, x)\right]=r(-\infty)
$$

The proof is complete.

The following theorem shows that if $c^{*}(\infty)>c \geq 0$, then the species persists in space and spreads to the right at the asymptotic spreading speed $c^{*}(\infty)$ and to the left at the asymptotic spreading speed $c^{*}(-\infty)$, forming a two-layer wave.

Theorem II.2 Assume that Hypotheses II.1 is satisfied and $c^{*}(\infty)>c \geq 0$. Assume also $u(t, x)$ is a solution of model (3) with $0<u(0, x) \leq r(\infty)$ for all $x$.

(a) If $u(0, x)$ is zero for all sufficiently large $x$, and $0 \leq u(0, x) \leq r(\infty)$, then for any positive $\epsilon$

$$
\lim _{t \rightarrow+\infty}\left[\sup _{x \geq t\left(c^{*}(\infty)+\epsilon\right)} u(t, x)\right]=0 .
$$

(b) If $0 \leq u(0, x) \leq r(\infty), u(0, x)>0$ on a closed interval, and $u(0, x)$ is zero for all sufficiently large $x$, then for every $\epsilon$ with $0<\epsilon<\frac{\left(c^{*}(\infty)-c\right)}{2}$

$$
\lim _{t \rightarrow+\infty}\left[\sup _{t(c+\epsilon) \leq x \leq t\left(c^{*}(\infty)-\epsilon\right)}|r(\infty)-u(t, x)|\right]=0 .
$$

(c) If $u(0, x)$ is zero for all sufficiently negative $x$, and $0 \leq u(0, x) \leq r(\infty)$, then for any positive $\epsilon$

$$
\lim _{t \rightarrow+\infty}\left[\sup _{x \leq-t\left(c^{*}(-\infty)+\epsilon\right)} u(t, x)\right]=0 .
$$

(d) If $u(0, x)>0$ on a closed interval and $0 \leq u(0, x) \leq r(\infty)$, then for every $\epsilon>0$

$$
\left.\lim _{t \rightarrow+\infty} \inf _{-t\left(c^{*}(-\infty)-\epsilon\right) \leq x \leq \min \left\{t(c-\epsilon), t\left(c^{*}(-\infty)-\epsilon\right)\right\}} u(t, x)\right]=r(-\infty)
$$

Remark II.2 Here we mention some of its biological implications in the theorem. Essentially, if the species' spreading speed in the high quality environment is sufficient to keep ahead of the rightward moving favorable habitat boundary, then the species will continue to expand ahead in the high quality environment at speed $c^{*}(\infty)$. Similarly the species will continue to expand leftward into the low quality environment at speed 
$c^{*}(-\infty)$. Statement (b) states that for regions behind the forward invasion front but sufficiently in front of the favorable habitat boundary, the population will essentially reach the high quality habitat equilibrium, $r(\infty)$. Similarly (d) states that for a region in front of the rearward invasion but sufficiently behind the favorable habitat boundary, the population will essentially achieve the equilibrium value of the poor quality habitat, $r(-\infty)$.

Proof. Firstly, we prove statement (a). From statement (a) in Lemma II.4, we have that for any positive $\epsilon$, there exist positive numbers $A$ and $\mu_{\epsilon}$, such that

$$
u(t, x) \leq A e^{-\mu_{\epsilon}\left(x-\left(c^{*}(\infty)+\frac{\epsilon}{2}\right) t\right)} .
$$

The trivial solution $u=0$ is a lower solution of model (3), so $u(t, x) \geq 0$ for all $t>0$ and $x$. It follows that

$$
\begin{aligned}
0 \leq \lim _{t \rightarrow+\infty}\left[\sup _{x \geq t\left(c^{*}(\infty)+\epsilon\right)} u(t, x)\right] & \leq \lim _{t \rightarrow+\infty}\left[\sup _{x \geq t\left(c^{*}(\infty)+\epsilon\right)} A e^{-\mu_{\epsilon}\left(x-\left(c^{*}(\infty)+\frac{\epsilon}{2}\right) t\right)}\right] \\
& \leq \lim _{t \rightarrow+\infty}\left[A e^{-\mu_{\epsilon}\left(t\left(c^{*}(\infty)+\epsilon\right)-\left(c^{*}(\infty)+\frac{\epsilon}{2}\right) t\right)}\right] \\
& =\lim _{t \rightarrow+\infty}\left[A e^{-\mu_{\epsilon} \frac{\epsilon}{2} t}\right]=0 .
\end{aligned}
$$

Therefore

$$
\lim _{t \rightarrow+\infty}\left[\sup _{x \geq t\left(c^{*}(\infty)+\epsilon\right)} u(t, x)\right]=0 .
$$

The proof of statement (b) is similar to the proof of statement(iii) in Theorem 2.2 of Li et al. (2015) and the proof of statement (c) is same as the proof of statement (b) in Theorem II.1, so we omit their proofs here.

Finally, we prove the statement (d). From the proof of statement (c) in Theorem II.1, we have that

$$
\lim _{t \rightarrow+\infty}\left[\inf _{-t\left(c^{*}(-\infty)-\epsilon\right) \leq x \leq t\left(c^{*}(-\infty)-\epsilon\right)} u(t, x)\right] \geq r(-\infty)
$$

In other words, for any given $\epsilon>0$, there exists $T_{0}$, such that

$$
u(t, x) \geq r(-\infty), \quad \text { if } t>T_{0}, \text { and }-t\left(c^{*}(-\infty)-\epsilon\right) \leq x \leq t\left(c^{*}(-\infty)-\epsilon\right)
$$


On the other hand, by using Lemma II.3, for the given $\epsilon>0$, there exists sufficiently large $T>0$ and $M>0$, such that,

$$
u(t, x) \leq r(-\infty)+\epsilon, \quad \text { if } t>T, \text { and } x \leq-M+c t
$$

If $c \leq c^{*}(-\infty)$, it follows from (36) and (37) that when $t>\max \left\{T, T_{0}, \frac{M}{\epsilon}\right\}$ and $-t\left(c^{*}(-\infty)-\epsilon\right) \leq x \leq t(c-\epsilon)$,

$$
r(-\infty) \leq u(t, x) \leq r(-\infty)+\epsilon
$$

If $c \geq c^{*}(-\infty)$, it follows from (36) and (37) that when $t>\max \left\{T, T_{0}, \frac{M}{c-c^{*}(-\infty)+\epsilon}\right\}$ and $-t\left(c^{*}(-\infty)-\epsilon\right) \leq x \leq t\left(c^{*}(-\infty)-\epsilon\right)$,

$$
r(-\infty) \leq u(t, x) \leq r(-\infty)+\epsilon
$$

Since $\epsilon$ can be any small positive number, we have that

$$
\lim _{t \rightarrow+\infty}\left[\inf _{-t\left(c^{*}(-\infty)-\epsilon\right) \leq x \leq \min \left\{t(c-\epsilon), t\left(c^{*}(-\infty)-\epsilon\right)\right\}} u(t, x)\right]=r(-\infty)
$$

The following theorem will show that when $c^{*}(-\infty)>-c>0$, the species persists in space and spreads to the right at the asymptotic spreading speed $c^{*}(\infty)$ and to the left at the asymptotic spreading speed $c^{*}(-\infty)$. The solution of model (3) forms a two layer wave.

Theorem II.3 Assume that Hypotheses II.1 is satisfied and $c^{*}(-\infty)>-c>0$. Assume also $u(t, x)$ is a solution of model (3) with $0<u(0, x) \leq r(\infty)$ for all $x$.

(a) If $u(0, x)$ is zero for all sufficiently large $x$, and $0 \leq u(0, x) \leq r(\infty)$, then for any positive $\epsilon$

$$
\lim _{t \rightarrow+\infty}\left[\sup _{x \geq t\left(c^{*}(\infty)+\epsilon\right)} u(t, x)\right]=0 .
$$

(b) If $0 \leq u(0, x) \leq r(\infty), u(0, x)>0$ on a closed interval, and $u(0, x)$ is zero for all sufficiently large $x$, then for every $\epsilon$ with $0<\epsilon<\frac{\left(c^{*}(\infty)-c\right)}{2}$

$$
\lim _{t \rightarrow+\infty}\left[\inf _{-t(-c-\epsilon) \leq x \leq t\left(c^{*}(\infty)-\epsilon\right)} u(t, x)\right]=r(\infty) .
$$


(c) If $u(0, x)$ is zero for all sufficiently negative $x$, and $0 \leq u(0, x) \leq r(\infty)$, then for any positive $\epsilon$

$$
\lim _{t \rightarrow+\infty}\left[\sup _{x \leq-t\left(c^{*}(-\infty)+\epsilon\right)} u(t, x)\right]=0 .
$$

(d) If $u(0, x)>0$ on a closed interval and $0 \leq u(0, x) \leq r(\infty)$, then for every $\epsilon>0$

$$
\lim _{t \rightarrow+\infty}\left[\inf _{\left.-t\left(c^{*}(-\infty)-\epsilon\right) \leq x \leq-t(-c+\epsilon)\right\}} u(t, x)\right]=r(-\infty) .
$$

Remark II.3 The theorem has some biological meanings. If the species spreading speed in the low quality environment is in excess of the leftward drift of the favorable habitat boundary, then the species will continue to spread leftward at a speed associated with the low quality habitat, $c^{*}(-\infty)$. The rightward expansion will be at the speed associated with the high quality environment, $c^{*}(\infty)$.

Proof. The proof for statement (a) and statement (c) are the same as those for statement (a) and statement (c) in Theorem II.2. We omit them here.

Now we prove statement (b). From statement (c) of Lemma II.11, for any small positive number $\epsilon$, there exist $\alpha, \gamma, \mu_{1}, \mu_{2}$, and a positive number $t_{0}$, such that when $t>t_{0}$,

$$
u(t, x) \geq w\left(\alpha, \mu_{1}, \mu_{2} ; t-t_{0}, x\right)
$$

where $\psi\left(\mu_{1}\right)=-c-\epsilon / 2$ and $\psi\left(\mu_{2}\right)=c^{*}(\infty)-\epsilon / 2$. If $u\left(t_{0}, x\right)$ is assumed to be the initial value for the model $(3)$, then the solution $u(t, x)$ satisfies the integral equation $(27)$.

Since when $t>t_{0}, x$ satisfies

$\ell-\sigma\left(\mu_{1}\right)-\psi\left(\mu_{1}\right)\left(t-t_{0}\right)+L \sqrt{4 d\left(t-t_{0}\right)} \leq x \leq \ell+3 \pi / \gamma+\sigma\left(\mu_{2}\right)+\psi\left(\mu_{2}\right)\left(t-t_{0}\right)-L \sqrt{4 d\left(t-t_{0}\right)}$,

and $y$ satisfies

$$
-L \sqrt{4 d\left(t-t_{0}\right)} \leq y \leq L \sqrt{4 d\left(t-t_{0}\right)}
$$

we have that

$$
\ell-\sigma\left(\mu_{1}\right)-\psi\left(\mu_{1}\right)\left(t-t_{0}\right) \leq x-y \leq \ell+3 \pi / \gamma+\sigma\left(\mu_{2}\right)+\psi\left(\mu_{2}\right)\left(t-t_{0}\right),
$$




$$
x-y-c t \geq \ell-\sigma\left(\mu_{1}\right)-\psi\left(\mu_{1}\right)\left(t-t_{0}\right)-c t=\ell-\sigma\left(\mu_{1}\right)+\epsilon / 2\left(t-t_{0}\right)-\epsilon / 2 t_{0} \geq \ell .
$$

Similar to the process in the proof of statement (c) of Theorem II.1, from (38)-(41) we have that

$$
u(t, x) \geq \tilde{u}^{(n)}(t)
$$

where $\tilde{u}^{(n)}(t)$ satisfies

$$
\begin{aligned}
\tilde{u}^{(n+1)}(t) & =(1-\epsilon) \alpha e^{-\rho\left(t-t_{0}\right)}+\int_{t_{0}}^{t} e^{-\rho(t-\tau)}(1-\epsilon) \tilde{u}^{(n)}(\tau)\left[\rho+r(\infty)-\epsilon-\frac{1}{1-\epsilon} \tilde{u}^{(n)}(\tau)\right] d \tau \\
& =a_{n}+b_{n}(t) e^{-\rho\left(t-t_{0}\right)}
\end{aligned}
$$

with,

$$
\begin{gathered}
a_{n}=\frac{(1-\epsilon) a_{n-1}\left(\rho+r(\infty)-\epsilon-a_{n-1}\right)}{\rho}, \\
a_{1}=\frac{(1-\epsilon) \alpha[\rho+r(\infty)-\epsilon-\alpha]}{\rho},
\end{gathered}
$$

and $b_{n}(t)$ is the sum of polynomials, and products of polynomials and exponential function in the form of $e^{-j \rho\left(t-t_{0}\right)}$ with $j$ a positive integer.

Since $\lim _{t \rightarrow \infty} \tilde{u}^{(n)}(t)=a_{n}$ and $\lim _{n \rightarrow \infty} a_{n}=r(\infty)-\epsilon-\epsilon \rho /(1-\epsilon)$, there exists a positive integer $N$ sufficiently large and $t_{2}>t_{0}$ such that for $t>t_{2}$

$$
\tilde{u}^{(N)}(t) \geq r(\infty)-\epsilon-\epsilon \rho /(1-\epsilon)
$$

Choose $t_{1}>t_{2}$ sufficiently large such that when $t>t_{1}$

$$
\ell-\sigma\left(\mu_{1}\right)-\psi\left(\mu_{1}\right)\left(t-t_{0}\right)+L N \sqrt{4 d\left(t-t_{0}\right)} \leq \ell+3 \pi / \gamma+\sigma\left(\mu_{2}\right)+\psi\left(\mu_{2}\right)\left(t-t_{0}\right)-L N \sqrt{4 d\left(t-t_{0}\right)}
$$

and also there exists $t_{3}>t_{1}$ such that when $t>t_{3}$

$$
\begin{array}{r}
\ell+\sigma\left(\mu_{1}\right)-\psi\left(\mu_{1}\right)\left(t-t_{0}\right)+L \sqrt{4 d\left(t-t_{0}\right)} \leq-t(-c-\epsilon) \leq t\left(c^{*}(\infty)-\epsilon\right), \\
t\left(c^{*}(\infty)-\epsilon\right) \leq \ell+3 \pi / \gamma+\sigma\left(\mu_{2}\right)+\psi\left(\mu_{2}\right)\left(t-t_{0}\right)-L N \sqrt{4 d\left(t-t_{0}\right)} .
\end{array}
$$

It follows from (42), (43) and (44) that

$$
\lim _{t \rightarrow \infty}\left[\inf _{t(c+\varepsilon) \leq x \leq t\left(c^{*}(\infty)-\varepsilon\right)} u(t, x)\right] \geq(1-\epsilon)(r(\infty)-\epsilon) .
$$


Since $\epsilon$ is arbitrarily small and $u(t, x) \leq r(\infty)$ for all $x$ and $t$, it follows that

$$
\lim _{t \rightarrow+\infty}\left[\inf _{-t\left(c^{*}(-\infty)-\epsilon\right) \leq x \leq t\left(c^{*}(-\infty)-\epsilon\right)} u(t, x)\right]=r(\infty) .
$$

Finally, we prove the statement (d). From statement (c) of Lemma II.11, for any small positive number $\epsilon$, there exist $\alpha, \gamma, \mu_{1}, \mu_{2}$, and a positive number $t_{0}$, such that when $t>t_{0}$

$$
u(t, x) \geq w_{l}\left(\alpha, \mu_{1}^{\prime}, \mu_{2}^{\prime} ; t-t_{0}, x\right)
$$

where $\psi\left(\mu_{1}^{\prime}\right)=c^{*}(-\infty)-\epsilon$ and $\psi\left(\mu_{2}^{\prime}\right)=-c+\epsilon$. Using the same method to prove the statement (c), we have that

$$
\lim _{t \rightarrow+\infty}\left[\inf _{\left.-t\left(c^{*}(-\infty)-\epsilon\right) \leq x \leq-t(-c+\epsilon)\right\}} u(t, x)\right] \geq r(-\infty) .
$$

Additionally, by using Lemma II.3, for the given $\epsilon>0$, there exists sufficiently large $T>0$ and $M>0$, such that, when $t>T$ and $x \leq-M+c t, u(t, x) \leq r(-\infty)+\epsilon$. Then when $t>\frac{M}{\epsilon}$, we have that $-t(-c+\epsilon)<-M+c t$, so that $u(t, x) \leq r(-\infty)+\epsilon$ for any $x$. Therefore,

$$
\lim _{t \rightarrow+\infty}\left[\inf _{\left.-t\left(c^{*}(-\infty)-\epsilon\right) \leq x \leq-t(-c+\epsilon)\right\}} u(t, x)\right]=r(-\infty) .
$$

The following theorem deals with when the habitat shifts rapidly leftward, $c^{*}(\infty) \geq-c>c^{*}(-\infty)$.

Theorem II.4 Assume that Hypotheses II.1 is satisfied and $c^{*}(\infty) \geq-c>c^{*}(-\infty)$. Assume also $u(t, x)$ is a solution of model (3) with $0<u(0, x) \leq r(\infty)$ for all $x$.

(a) If $u(0, x)$ is zero for all sufficiently large $x$, and $0 \leq u(0, x) \leq r(\infty)$, then for any positive $\epsilon$

$$
\lim _{t \rightarrow+\infty}\left[\sup _{x \geq t\left(c^{*}(\infty)+\epsilon\right)} u(t, x)\right]=0 .
$$

(b) If $u(0, x)>0$ on a closed interval and $0 \leq u(0, x) \leq r(\infty)$, then for every $\epsilon$ satisfying $0<\epsilon<\frac{c^{*}(\infty)-\epsilon}{3}$,

$$
\lim _{t \rightarrow+\infty}\left[\inf _{t(c+\epsilon) \leq x \leq t\left(c^{*}(\infty)-\epsilon\right)} u(t, x)\right]=r(\infty) .
$$


(c) If $u(0, x)$ is zero for all sufficiently negative $x$, and $0 \leq u(0, x) \leq r(\infty)$, then for any positive $\epsilon$

$$
\lim _{t \rightarrow+\infty}\left[\sup _{x \leq-t(-c+\epsilon)} u(t, x)\right]=0 .
$$

Remark II.4 Biologically if the leftward moving speed of the favorable habitat boundary, $|c|$, exceeds the spreading speed in the low quality habitat, but is lower than the spreading speed in the high quality habitat, then the species will effectively only exist in the high quality habitat. Its leftward spreading speed will be $|c|$ and rightward spreading speed will be $c^{*}(\infty)$.

Proof. Statement (a) is obvious, so we omit the details of the proof here. The proof of statement (b) is similar to the proof of statement (c) in Theorem II.1 with lower solution defined in statement $(\mathrm{d})$ of Lemma II.11, which is that when $t>t_{0}$

$$
u(t, x) \geq w\left(\alpha, \mu_{1}, \mu_{2} ; t-t_{0}, x\right)
$$

where $\psi\left(\mu_{1}\right)=-c-\epsilon$ and $\psi\left(\mu_{2}\right)=c^{*}(\infty)-\epsilon$. We omit the details of the proof here.

Now we are going to prove the statement (c). From the formula (11), for the given $\epsilon$, there exists $\delta>0$ and $0<\mu_{3}<\mu^{*}(-\infty)$, such that $\mu_{3}\left(c^{*}(-\infty)+\epsilon / 2\right)=$ $d \mu_{3}^{2}+r(-\infty)+\delta$. We assume that $u_{3}(t, x)=A_{3} e^{\mu_{3}\left(x+\left(c^{*}(-\infty)+\epsilon\right) t\right)}$. Let $x_{0}$ satisfies that for any $x<x_{0}, r(x)<r(-\infty)+\delta, u(0, x) \leq u_{3}(0, x)=A_{3} e^{\mu_{3} x}$ and $b$ is the number introduced in Lemma II.5 with $\mu=\mu_{3}$. Assume the sequence $u^{(n)}(t, x)$ is defined by the formula (7) with $u^{(0)}(t, x)=0$. Obviously, $u^{(0)}(t, x)=0$ is a lower solution of system (3) and $u(t, x)=\lim _{n \rightarrow \infty} u^{(n)}(t, x)$. When $x \leq x_{0}+c t-b$, we have that

$$
\begin{aligned}
u^{(1)}(t, x) & =Q\left[u^{(0)}\right](t, x)=\int_{-\infty}^{+\infty} k(t, x-y) u_{0}(y) d y \\
& =A_{3} e^{-\rho t+\mu_{3} x+d \mu_{3}^{2} t} \\
& =u_{3}(t, x) e^{-\rho t-\left(r(-\infty)+\delta+\frac{\mu_{3} \epsilon}{2}\right) t} \leq u_{3}(t, x) .
\end{aligned}
$$

Since

$$
\begin{aligned}
Q\left[u_{3}\right](t, x)= & \int_{-\infty}^{+\infty} k(t, x-y) u_{0}(y) d y+ \\
& \int_{0}^{t} \int_{-\infty}^{+\infty} k(t-\tau, x-y) u_{3}(\tau, y)\left[\rho+r(y-c \tau)-u_{3}(\tau, y)\right] d y d \tau
\end{aligned}
$$




$$
\begin{aligned}
\leq & \int_{-\infty}^{+\infty} k(t, y) A_{3} e^{\mu_{3}(x-y)} d y+ \\
& \int_{0}^{t} \int_{-\infty}^{+\infty} k(\tau, y) A_{3} e^{\mu_{3}\left(x-y+\left(c^{*}(-\infty)+\epsilon\right)(t-\tau)\right)}[\rho+r(x-y-c(t-\tau))] d y d \tau \\
\leq & \int_{-\infty}^{+\infty} k(t, y) A_{3} e^{\mu_{3}(x-y)} d y+\left(1+S_{0} \epsilon_{1}\right) \cdot \\
& \int_{0}^{t} \int_{c \tau-b}^{+\infty} k(\tau, y) A_{3} e^{\mu_{3}\left(x-y+\left(c^{*}(-\infty)+\epsilon\right)(t-\tau)\right)}[\rho+r(x-y-c(t-\tau))] d y d \tau \\
\leq & \int_{-\infty}^{+\infty} k(t, y) A_{3} e^{\mu_{3}(x-y)} d y+\left(1+S_{0} \epsilon_{1}\right)(\rho+r(-\infty)+\delta) \cdot \\
& \int_{0}^{t} \int_{c \tau-b}^{+\infty} k(\tau, y) A_{3} e^{\mu_{3}\left(x-y+\left(c^{*}(-\infty)+\epsilon\right)(t-\tau)\right)} d y d \tau \\
\leq & \int_{-\infty}^{+\infty} k(t, y) A_{3} e^{\mu_{3}(x-y)} d y+(\rho+r(-\infty)+\delta)\left(1+S_{0} \epsilon_{1}\right) \cdot \\
& \int_{0}^{t} e^{\mu_{3}\left(c^{*}(-\infty)+\epsilon\right)(t-\tau)} \int_{-\infty}^{+\infty} k(\tau, y) A_{3} e^{\mu_{3}(x-y)} d y d \tau \\
\leq & A_{3} e^{-\rho t+\mu_{3} x+d \mu_{3}^{2} t}+(\rho+r(-\infty)+\delta)\left(1+S_{0} \epsilon_{1}\right) \cdot \\
& \int_{0}^{t} e^{\mu_{3}\left(c^{*}(-\infty)+\epsilon\right)(t-\tau)} A_{3} e^{-\rho \tau+\mu_{3} x+d \mu_{3}^{2} \tau} d \tau \\
= & A_{3} e^{-\rho t+\mu_{3} x+d \mu_{3}^{2} t}+\frac{\rho+r(-\infty)+\delta}{d \mu_{3}^{2}-\rho-\mu_{3}\left(c^{*}(-\infty)+\epsilon\right)}\left(1+S_{0} \epsilon_{1}\right) \cdot \\
& A e^{\mu_{3}\left(x+\left(c^{*}(-\infty)+\epsilon\right) t\right)}\left(e^{\left(d \mu_{3}^{2}-\rho-\mu_{3}\left(c^{*}(-\infty)+\epsilon\right) t\right.}-1\right) \\
= & u_{3}(t, x) .
\end{aligned}
$$

By induction, $u^{(n)}(t, x) \leq u_{3}(t, x)$. Since $u^{(n)}(t, x)$ converges to $u(t, x)$, so $u(t, x) \leq$ $u_{3}(t, x)$ for any $x \leq x_{0}+c t-b$.

Since when $t>T_{\epsilon}=\frac{\left(b-x_{0}\right)}{\epsilon},(c-\epsilon) t \leq x_{0}+c t-b$, we have that

$$
\begin{aligned}
0 \leq \lim _{t \rightarrow+\infty}\left[\sup _{x \leq-t(-c+\epsilon)} u(t, x)\right] & \leq \lim _{t \rightarrow+\infty}\left[\sup _{x \leq-t(-c+\epsilon)} A_{3} e^{\mu_{3}\left(x+\left(c^{*}(-\infty)+\epsilon\right) t\right)}\right] \\
& \leq \lim _{t \rightarrow+\infty}\left[A_{3} e^{\mu_{3}\left(t(c-\epsilon)-\left(c^{*}(-\infty)+\epsilon\right) t\right)}\right] \\
& =\lim _{t \rightarrow+\infty}\left[A_{3} e^{\left.\mu_{3}\left(c+c^{*}(-\infty)\right) t\right)}\right]=0
\end{aligned}
$$

The proof is complete.

The following theorem deals with when $c^{*}(\infty) \leq-c$, so the habitat shifts extremely rapidly leftward.

Theorem II.5 Assume that Hypotheses II.1 is satisfied and $c^{*}(\infty) \leq-c$. Assume also $u(t, x)$ is a solution of model (3) with $0<u(0, x) \leq r(\infty)$ for all $x$. 
(a) If $u(0, x)$ is zero for all sufficiently large $x$, and $0 \leq u(0, x) \leq r(\infty)$, then for any positive $\epsilon$

$$
\lim _{t \rightarrow+\infty}\left[\sup _{x \geq t\left(c^{*}(\infty)+\epsilon\right)} u(t, x)\right]=0
$$

(b) If $u(0, x)>0$ on a closed interval and $0 \leq u(0, x) \leq r(\infty)$, then for every $\epsilon$ satisfying $0<\epsilon<\frac{c^{*}(\infty)-\epsilon}{3}$,

$$
\lim _{t \rightarrow+\infty}\left[\inf _{-t\left(c^{*}(\infty)-\epsilon\right) \leq x \leq t\left(c^{*}(\infty)-\epsilon\right)} u(t, x)\right]=r(\infty) .
$$

(c) If $u(0, x)$ is zero for all sufficiently negative $x$, and $0 \leq u(0, x) \leq r(\infty)$, then for any positive $\epsilon$

$$
\lim _{t \rightarrow+\infty}\left[\sup _{x \leq-t\left(c^{*}(\infty)+\epsilon\right)} u(t, x)\right]=0 .
$$

Remark II.5 Biologically, if the leftward moving speed of the favorable habitat boundary is extremely large, then the species will effectively only exist in the high quality habitat. The species will persist and spread in both directions in the habitat with asymptotic spreading speed $c^{*}(\infty)$. The lower solution defined in statement (e) of Lemma II.11 can be used to prove this theorem. The proof is similar to Theorem II.4, so we only provide the statement of the theorem without proof here.

6 Simulations for One Species Models

In this section, we present some numerical simulations for the model (3) with

$$
r(x)=\left\{\begin{aligned}
1 & \text { if } x>0 \\
0.5 & \text { otherwise }
\end{aligned}\right.
$$

and the initial data

$$
u(0, x)=\left\{\begin{aligned}
0.5 \sin (x) & \text { if } 0<x<\pi \\
0 & \text { otherwise }
\end{aligned}\right.
$$

The species was initially introduced on the interval $[0, \pi]$. The numerical simulations with $r(-\infty)<0$ were provided in Li et al. (2015). Here we show numerically how 
$c$ affects the spatial dynamics of model (3) when $r(x)>0$ for all of $x$. Numerical simulations were conducted using MATLAB.

Choose $d=2$, so that, for model $(3), c^{*}(\infty)=2.8284 \ldots, c^{*}(-\infty)=2$ and $\underline{c}=4.8284 \ldots$. In each of the simulation figures in this section, the red dashed curve is the graph of $r(x-c t)$ when $t=0$. The travel direction of growth rate function $r(x-c t)$ is indicated with the arrow. The solid curves with different colors are used to describe the densities of the species at time $t$, for all of $x \in \mathrm{R}$. The speed of the species spread in both directions is numerically calculated by using appropriate level sets, which are close to the analytical spreading speeds. The speed $c$ and the numerical spreading speeds are provided on the top of the direction arrows.

Figure 1a displays the numerical solution with $c=3$, which satisfies $c^{*}(\infty)<$ $c<\underline{c}$. We see the species is not able to keep up with the high quality habitat, and thus effectively only experiences the low quality habitat and spreads in both directions at speed $c^{*}(-\infty)$. In the case of $c>\underline{c}$, the numerical solution is similar. This numerical result supports Theorem II.1.

Figure $1 \mathrm{~b}$ describes the numerical solution with $c=1$, so $c^{*}(\infty)>c>0$. The species is able to spread into the high quality habitat on the right at the asymptotic spreading speed $c^{*}(\infty)$ and spreads to the left at the asymptotic spreading speed $c^{*}(-\infty)$. The species distributes as a two-step hierarchy. This numerical result supports Theorem II.2.

Figure 1c provides us the numerical solution for $c=-1<0$, which satisfies $c^{*}(-\infty)>-c$. The species is able to keep up with the leftward moving boundary and expand into the poor quality habitat at $c^{*}(-\infty)$ and expand into the high quality habitat at $c^{*}(\infty)$. This numerical result supports Theorem II.3.

In Figure 1d, $c=-3<0$, and $c^{*}(\infty)<-c$. The species is not able to expand into the fast leftward moving low quality habitat, and effectively only experiences the high quality habitat. The species persists in space and spreads both to the right and left at the asymptotic spreading speed $c^{*}(\infty)$. In the case of $c^{*}(-\infty)<-c<c^{*}(\infty)$, the species persists in space and spreads to the right at the asymptotic spreading speed $c^{*}(\infty)$ and to the left at speed $c$. This numerical result supports Theorem II.4 
and Theorem II.5.

\section{Discussion for Reaction-Diffusion Chapter}

In this chapter, we used a reaction-diffusion model to study the persistence and spread of a species along shifting resource gradients. We considered that the region suitable for species growth is expanding or contracting as well as assuming that the species grows everywhere along the spatial gradient, but with differing levels of success. We can summarize the key results as follows:

- When the favorable habitat for species growth is contracting, the rightward spreading speed is $c^{*}(\infty)$ if the contracting speed $c$ is relatively small and is $c^{*}(-\infty)$ if the contracting speed $c$ is relatively large. In this case, the leftward spreading speed is $c^{*}(-\infty)$.

- When the favorable habitat for species growth is expanding, the rightward spreading speed is $c^{*}(\infty)$, while the leftward spreading speed depends on who fast climate change is occurring relative to the intrinsic rate of spread of invasive species. We showed that the leftward spreading speed is $c^{*}(\infty)$ for relatively rapidly expansion, $|c|$ itself for relatively intermediate expansion, and $c^{*}(-\infty)$ for relatively slow expansion.

- The solution of the reaction-diffusion equation can create a two-layer wave with analytically determined propagation speeds, which was not found in the work of Li et al. (2015).

This extends the work by Li et al. (2015) to study the the impact of an expanding/contracting habitat edge on the persistence and spread of an invasive species. In this model the region suitable for species growth was assumed to be contracting and $r(-\infty)$ was assumed negative.

This work complements ( $\mathrm{Li}$ et al. 2016) which modeled a similar shifting resource gradient but with an integral-difference model. 


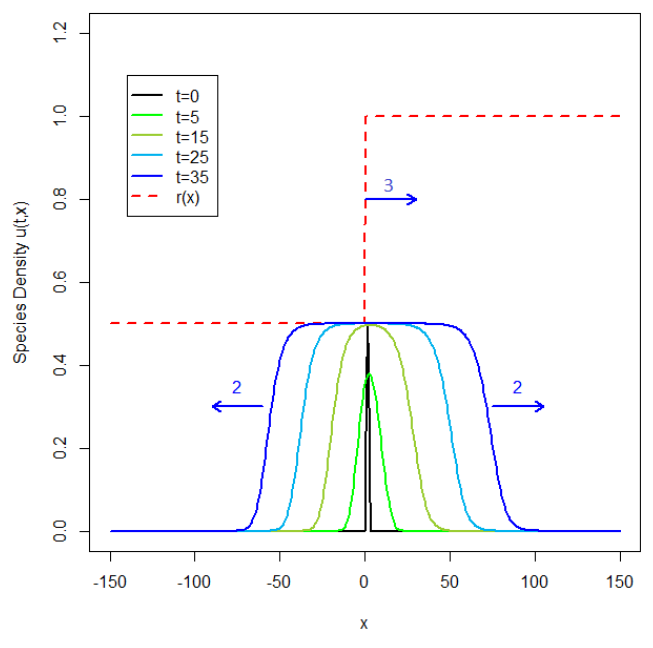

(a) $c=3$

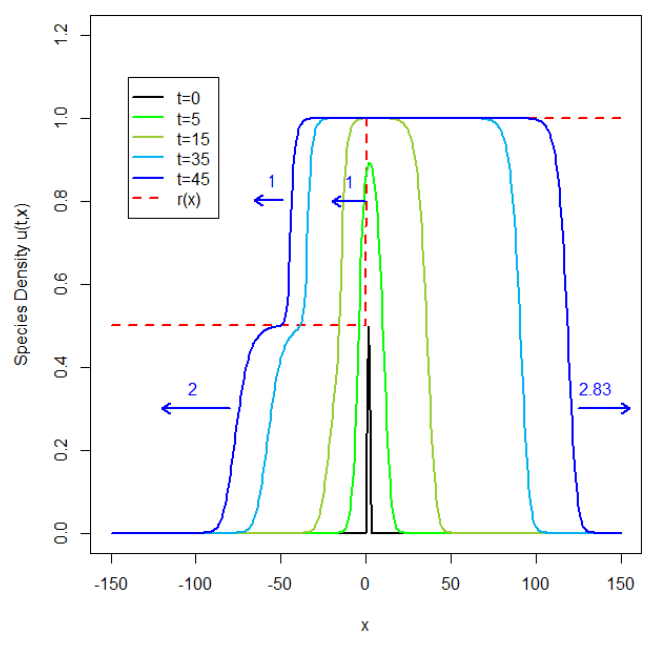

(c) $c=-1$

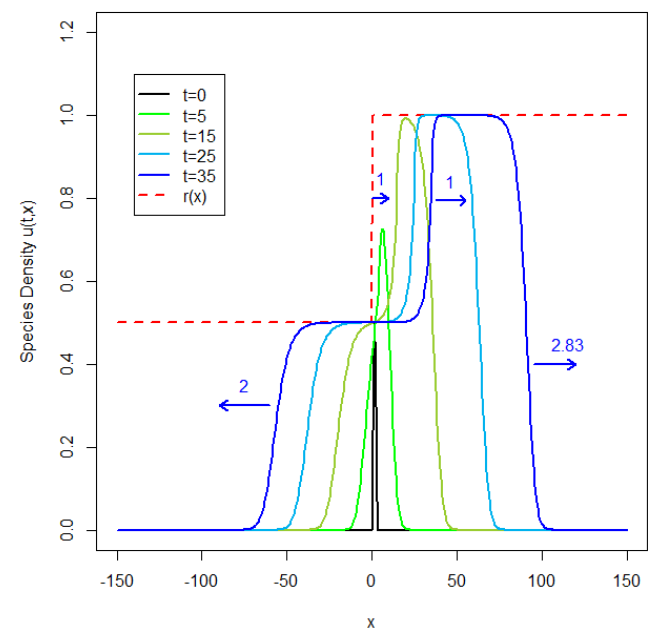

(b) $c=1$

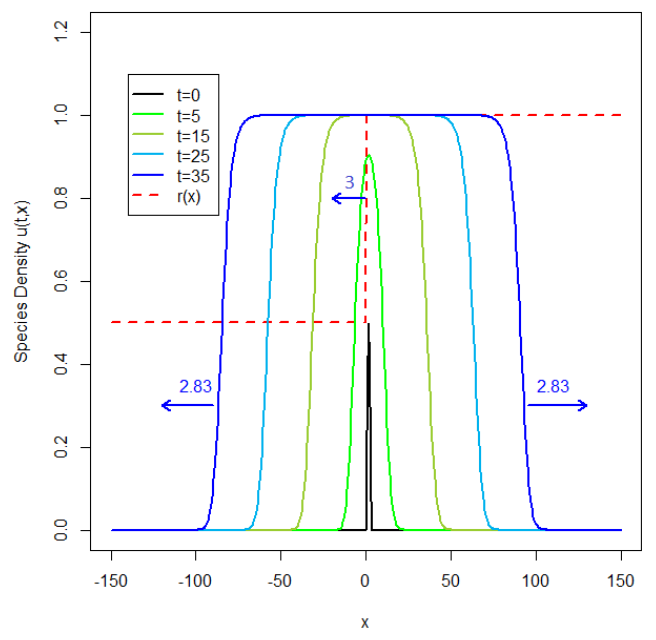

(d) $c=-3$

Figure 1: Numerical Simulation for Reaction-Diffusion Equations 


\section{CHAPTER III}

\section{LOTKA-VOLTERRA COMPETITION MODEL ANALYSIS}

\section{Lotka-Volterra Competition Model}

The competitive Lotka-Volterra model is a simple model to analyze the population dynamics of two or more species which are competing for some common limiting resource. This means the demand on the resource is greater than the supply, for example, food, nutrients, space, and nesting sites. The stronger competitor in the system will affect the population size and growth rate of other competitors, which in turn influences population dynamics. It has been demonstrated that the LotkaVolterra model could be utilized to sponsor beneficial invasions to control selected pest problems and stem the invasion of introduced pests (Baker 1990; Lewis 1996).

One simple example of the Lotka-Volterra competition model may take the form(Kan-on 1996; Morita 2009; Lewis 2002; Li 2005):

$$
\left\{\begin{array}{l}
\frac{\partial u}{\partial t}=d_{1} \frac{\partial^{2} u}{\partial x^{2}}+r_{1} u-u^{2}-a_{1} u v \\
\frac{\partial v}{\partial t}=d_{2} \frac{\partial^{2} v}{\partial x^{2}}+r_{2} v-v^{2}-a_{2} u v .
\end{array}\right.
$$

Here $u(t, x)$ and $v(t, x)$ represent the densities of the competing species at time $t$, and location $x$. Each species is assumed to have a linear growth term, a quadratic term for self-competition as well as a term for the interaction with the other species. The competitive interactions between two species may have a very important impact for the persistence and spread of both species. The densities of both competing species are required to be nonnegative and the parameters in this model $d_{1}, d_{2}, r_{1}, r_{2}, a_{1}$ and $a_{2}$ are all positive constants.

In general, the model (45) has four constant equilibria: the unpopulated state $(0,0)$; the first-species monoculture state $\left(r_{1}, 0\right)$; the second-species monoculture state 
$\left(0, r_{2}\right)$; and the coexistence state $\left(u^{*}, v^{*}\right)$, where

$$
u^{*}=\frac{r_{1}-r_{2} a_{1}}{1-a_{1} a_{2}}, v^{*}=\frac{r_{2}-r_{1} a_{2}}{1-a_{1} a_{2}} .
$$

The dynamics of the Lotka-Volterra competition model is more complex than the one dimension reaction-diffusion equation. Some well-known results for a scalar reactiondiffusion equation can be extended to the reaction system, but care must be taken with the assumptions. The uniqueness and existence of the solution for system (45) have been provided in Pao (1998).

The dynamics of population spread and traveling waves can be extended beyond reaction-diffusion models to multi-species systems(Lewis 2002; Weinberger 1982; Lui 1989a; Lui 1989b; Aronson and Weinberger 1975; Aronson and Weinberger 1978). Lui showed how to define the spreading speed of a class of cooperative systems in population ecology and epidemic theory and established the existence of a single spreading speed $c^{*}$ for all species. The spreading speed of one species invading habitat inhabited by another species has been demonstrated to be the slowest speed of a class of traveling waves. This result was generalized by Weinberger (2002) and introduced a new possibility - different species may have different spreading speeds, so that there are a slowest speed $c^{*}$ and a fastest speed $c_{f}^{*}$. Li et al. (2005) showed that, for a large class of multi-species systems, the slowest spreading speed $c^{*}$ is always characterized as the slowest speed of a class of traveling wave solutions. These traveling waves connect the unstable mono-culture equilibrium to the coexistence equilibrium or to the other mono-culture equilibrium. Since the two-species competition model can be converted into cooperative models by a simple change of variables, as an extension of work by Weinberger (2002) and Lui (1989a), Lewis (2002) provided an analysis of the spreading speed and linear determinacy for two-species competition models.

\section{Lotka-Volterra Competition Model with Shifting Habitat}

To explore the impact of climate change for the persistence and spread of two competing populations, our analysis in this chapter is mainly based on the Lotka- 
Volterra model with shifting habitat as follows:

$$
\left\{\begin{array}{l}
\frac{\partial u}{\partial t}=d_{1} \frac{\partial^{2} u}{\partial x^{2}}+r_{1}(x-c t) u-u^{2}-a_{1} u v \\
\frac{\partial v}{\partial t}=d_{2} \frac{\partial^{2} v}{\partial x^{2}}+r_{2}(x+c t) v-v^{2}-a_{2} u v .
\end{array}\right.
$$

Here $u=u(t, x)$ and $v=v(t, x)$ are the densities of two competing populations. The parameters $d_{1}>0$ and $d_{2}>0$ are the diffusion constants and $a_{1}>0$ and $a_{2}>0$ are the coefficients describing competitions between the species. In this system, $r_{1}(x-c t)$ represents the intrinsic growth rate function of the species $u$ and $r_{2}(x+c t)$ represents the intrinsic growth rate function of the species $v$. To consider the effect of climate change on population dynamics, we assume that both species are initially introduced in a bounded area.

We begin our discussion by making the following hypothesis for $r_{i}(x)$, with $i=1,2$.

Hypothesis III.1 $r_{1}(x)$ is a locally Holder nondecreasing, bounded, and piecewise continuously differentiable function in $x$ for $-\infty<x<\infty, 0<r_{1}(\infty)<\infty$, and $-\infty<r_{1}(-\infty)<0 ; r_{2}(x)$ is a locally Holder nonincreasing, bounded, and piecewise continuously differentiable function in $x$ for $-\infty<x<\infty, 0<r_{2}(-\infty)<\infty$, and $-\infty<r_{2}(\infty)<0$.

Since $r_{1}$ has a sign change, the domain can be portioned into two regions. The region where $r_{1}(x-c t)<0$ will be unsuitable for the growth of $u$, and the region where $r_{1}(x-c t)>0$ is the region in which $u$ can grow. Similarly, the domain of species $v$ can be portioned into a suitable habitat and an unsuitable habitat as well. In this chapter, we assume that $c>0$ so that the edge of the habitat suitable for species $u$ is moving in the positive direction and the edge of the habitat suitable for species $v$ is moving in the negative direction at speed $c$. That means, the suitable habitat for both species are contracting.

In discussing the Lotka-Volterra model (46), we need to expand the monotone property of the reaction function to competition models. We define the function $f_{i}(x, y, u, v)$ with $i=1,2$ to be quasimonotone nondecreasing(resp., nonincreasing) in $u$ (resp., $v$ ) if for fixed $v$ (resp., $u$ ), $f$ is nondecreasing (resp., nonincreasing) in $u$ 
(resp., $v$ ). Hence for the coupled reaction-diffusion system, there are three basic types of quasimonotone functions.

Definition III.1 A function $f=\left(f_{1}, f_{2}\right)$ is called quasimonotone nondecreasing (resp., nonincreasing) in $R \times R$ if both $f_{1}$ and $f_{2}$ are quasimonotone nondecreasing (resp., nonincreasing) for $(u, v) \in R \times R$. When $f_{1}$ is quasimonotone nonincreasing and $f_{2}$ is quasimonotone nondecreasing (or vice versa), then $f$ is called mixed quasimonotone.

For the Lotka-Volterra model (46), we assume that

$$
\left(f_{1}, f_{2}\right)=\left(r_{1}(x-c t) u-u^{2}-a_{1} u v, r_{2}(x+c t) v-v^{2}-a_{2} u v\right) .
$$

We have that

$$
\frac{\partial f_{1}}{\partial v}=-a_{1} u \leq 0 ; \frac{\partial f_{2}}{\partial u}=-a_{2} v \leq 0
$$

so that $\left(f_{1}, f_{2}\right)$ is quasimonotone nonincreasing. Then we can extend the monotone method for scalar problems to the coupled system (46) using upper and lower solutions as the initial iterations. We have the following definition of ordered upper and lower solutions, which were given in Pao (1992).

Definition III.2 A pair of functions $\tilde{\mathrm{u}}=(\tilde{u}, \tilde{v}), \hat{\mathrm{u}}=(\hat{u}, \hat{v})$ in $\mathrm{C}^{1,2}([0, \infty] \times[-\infty, \infty])$ are called ordered upper and lower solutions of model (46) with initial values $u(0, x)=$ $u_{0}(x)$ and $v(0, x)=v_{0}(x)$ if they satisfy the relation $\tilde{\mathrm{u}} \geq \hat{\mathrm{u}}$ and

$$
\begin{aligned}
& \tilde{u}(0, x) \geq u_{0}(x) \geq \hat{u}(0, x) \\
& \tilde{v}(0, x) \geq v_{0}(x) \geq \hat{v}(0, x),
\end{aligned}
$$

and if

$$
\begin{aligned}
& \frac{\partial \tilde{u}}{\partial t}-d_{1} \frac{\partial^{2} \tilde{u}}{\partial x^{2}}-f_{1}(t, x, \tilde{u}, \hat{v}) \geq 0 \geq \frac{\partial \hat{u}}{\partial t}-d_{1} \frac{\partial^{2} \hat{u}}{\partial x^{2}}-f_{1}(t, x, \hat{u}, \tilde{v}) \\
& \frac{\partial \tilde{v}}{\partial t}-d_{2} \frac{\partial^{2} \tilde{v}}{\partial x^{2}}-f_{2}(t, x, \hat{u}, \tilde{v}) \geq 0 \geq \frac{\partial \hat{v}}{\partial t}-d_{2} \frac{\partial^{2} \hat{v}}{\partial x^{2}}-f_{2}(t, x, \tilde{u}, \hat{v})
\end{aligned}
$$

when $\left(f_{1}, f_{2}\right)$ is quasimonotone nonincreasing.

It is easy to show that $\tilde{\mathrm{u}}=\left(r_{1}(\infty), r_{2}(-\infty)\right), \hat{\mathrm{u}}=(0,0)$ are ordered upper and lower solutions and $\hat{\mathrm{u}}$ is the trivial solution of the system (46). For every $\left(u_{1}, v_{1}\right)$ and 
$\left(u_{2}, v_{2}\right)$, which satisfies $0 \leq u_{i} \leq r_{1}(\infty)$ and $0 \leq v_{i} \leq r_{2}(-\infty)$ for $i=1,2$, we have that

$$
\left|f_{1}\left(t, x, u_{1}, v_{1}\right)-f_{1}\left(t, x, u_{2}, v_{1}\right)\right| \leq\left(3 r_{1}(\infty)+a_{1} r_{2}(-\infty)\right)\left|u_{1}-u_{2}\right|
$$

and

$$
\left|f_{2}\left(t, x, u_{1}, v_{1}\right)-f_{2}\left(t, x, u_{1}, v_{2}\right)\right| \leq\left(3 r_{2}(-\infty)+a_{2} r_{1}(\infty)\right)\left|v_{1}-v_{2}\right|,
$$

so that $\left(f_{1}, f_{2}\right)$ satisfies the Lipschitz Conditions. Define the function

$$
\begin{aligned}
& F_{1}(t, x, u, v)=\beta u+f_{1}(t, x, u, v) \\
& F_{2}(t, x, u, v)=\beta v+f_{2}(t, x, u, v),
\end{aligned}
$$

where $\beta>\max \left\{3 r_{1}(\infty)+a_{1} r_{2}(-\infty), 3 r_{2}(-\infty)+a_{2} r_{1}(\infty)\right\}$. Then $F_{1}$ and $F_{2}$ are Holder continuous and monotone nondecreasing in $u$ and $v$ separately. By adding dominant linear terms to both sides of the equations in system (46), we obtain the equivalent equation of (46):

$$
\left\{\begin{array}{l}
\frac{\partial u}{\partial t}+\beta u=d_{1} \frac{\partial^{2} u}{\partial x^{2}}+\beta u+r_{1}(x-c t) u-u^{2}-a_{1} u v \\
\frac{\partial v}{\partial t}+\beta v=d_{2} \frac{\partial^{2} v}{\partial x^{2}}+\beta v+r_{2}(x+c t) v-v^{2}-a_{2} u v
\end{array}\right.
$$

From Pao (1992), the solution of system (47) with $u(0, x)=u_{0}(x)$ and $v(0, x)=v_{0}(x)$ satisfies the integral equation as follows:

$$
\left\{\begin{aligned}
u(t, x)= & Q_{1}[(u, v)](t, x)=\int_{-\infty}^{+\infty} k_{1}(t, x-y) u_{0}(y) d y \\
& +\int_{0}^{t} \int_{-\infty}^{+\infty} k_{1}(t-\tau, x-y) u(\tau, y)\left[\beta+r_{1}(y-c \tau)-u(\tau, y)-a_{1} v(\tau, y)\right] d y d \tau \\
v(t, x)= & Q_{2}[(u, v)](t, x)=\int_{-\infty}^{+\infty} k_{2}(t, x-y) v_{0}(y) d y \\
& +\int_{0}^{t} \int_{-\infty}^{+\infty} k_{2}(t-\tau, x-y) v(\tau, y)\left[\beta+r_{2}(y+c \tau)-v(\tau, y)-a_{2} u(\tau, y)\right] d y d \tau,
\end{aligned}\right.
$$

where

$$
k_{i}(s, y)=\frac{1}{\sqrt{4 \pi d_{i} s}} e^{-\beta s-\frac{y^{2}}{4 d_{i} s}}, \quad \text { with } i=1,2 .
$$

Then the system (46) is also equivalent to the system as follows:

$$
\left(u^{(n+1)}, v^{(n+1)}\right)=Q\left[\left(u^{(n)}, v^{(n)}\right)\right]
$$


where $Q=\left(Q_{1}, Q_{2}\right), u^{(0)}(t, x)$ and $v^{(0)}(t, x)$ are the given ordered upper and lower solutions and $\left(u^{(n)}(t, x), v^{(n)}(t, x)\right)$ are defined by

$$
\left\{\begin{array}{l}
u^{(n+1)}(t, x)=Q_{1}\left[\left(u^{(n)}, v^{(n)}\right)\right](t, x) \\
v^{(n+1)}(t, x)=Q_{2}\left[\left(u^{(n)}, v^{(n)}\right)\right](t, x) .
\end{array}\right.
$$

Theory on the existence-comparison theorem for quasimonotone nonincreasing functions has been well established (e.g. Theorem 8.3.2 in Pao (1992, page 397)). This theory shows that the sequence $\left\{\bar{u}^{(k)}, \underline{v}^{(k)}\right\}$ and $\left\{\underline{u}^{(k)}, \bar{v}^{(k)}\right\}$ obtained from system (48) with $\left\{\bar{u}^{(0)}, \underline{v}^{(0)}\right\}=\left(r_{1}(\infty), 0\right)$ and $\left\{\underline{u}^{(k)}, \bar{v}^{(k)}\right\}=\left(0, r_{2}(-\infty)\right)$ converges monotonically to $(u, v)$, which is the unique solution between $\tilde{\mathrm{u}}$ and $\hat{\mathrm{u}}$ of the problem (46) with initial values between $\tilde{u}$ and $\hat{u}$. The monotone property of the sequence is in the sense of $\underline{u}^{(k)} \leq \underline{u}^{(k+1)} \leq \bar{u}^{(k+1)} \leq \bar{u}^{(k)}$, and $\underline{v}^{(k)} \leq \underline{v}^{(k+1)} \leq \bar{v}^{(k+1)} \leq \bar{v}^{(k)}$.

To consider the spreading speed of the Lotka-Volterra competition model, we introduce some notations. Let

$$
\phi_{i}\left(x ; \mu_{i}\right)=\frac{d \mu_{i}^{2}+r_{i}(x)}{\mu} \text { and } \psi_{i}(\mu)=2 d_{i} \mu_{i},
$$

where $i=1,2$. When $\mu_{i}=\mu_{i}^{*}(x)=\sqrt{\frac{r_{i}(x)}{d_{i}}}, \phi_{i}\left(x ; \mu_{i}\right)$ get its minimum

$$
c_{i}^{*}(x)=\inf _{\mu_{i}>0} \phi\left(x ; \mu_{i}\right)=2 \sqrt{d_{i} r_{i}(x)} .
$$

It is easily seen that $\phi_{i}\left(x ; \mu_{i}\right)>\psi_{i}\left(\mu_{i}\right)$ for $0<\mu_{i}<\mu_{i}^{*}(x)$ and $\phi_{i}\left(x ; \mu_{i}^{*}(x)\right)=$ $\psi_{i}\left(\mu_{i}^{*}(x)\right)$.

\section{Non-persistence}

In this section, we will prove that, if the region suitable for each competition species contracts relatively fast, then both species will become extinct in the long run. We first provide a useful lemma for equation (46).

Lemma III.1 Let $(u(t, x), v(t, x))$ be the solution of system (46) with initial value $0 \leq u(0, x) \leq r_{1}(\infty)$ and $0 \leq v(0, x) \leq r_{2}(-\infty)$. For any positive $\epsilon$, there exist $T>0$ and $M>0$, such that

$$
\begin{gathered}
u(t, x)<\epsilon \text { for } t>T \text { and } x \leq-M+c t, \\
v(t, x)<\epsilon \text { for } t>T \text { and } x \geq M-c t .
\end{gathered}
$$


Proof. Let $(\dot{u}(t, x), \dot{v}(t, x))$ be the solution of the model as follows:

$$
\left\{\begin{array}{l}
\frac{\partial u}{\partial t}=d_{1} \frac{\partial^{2} u}{\partial x^{2}}+r_{1}(x-c t) u-u^{2} \\
\frac{\partial v}{\partial t}=d_{2} \frac{\partial^{2} v}{\partial x^{2}}+r_{2}(x+c t) v-v^{2} .
\end{array}\right.
$$

In this model, the species $\dot{u}(t, x)$ and $\dot{v}(t, x)$ satisfy

$$
\left\{\begin{array}{l}
\frac{\partial \dot{u}}{\partial t} \geq d_{1} \frac{\partial^{2} \dot{u}}{\partial x^{2}}+r_{1}(x-c t) \dot{u}-\dot{u}^{2}-a_{1} \dot{u} \dot{v} \\
\frac{\partial \dot{v}}{\partial t} \geq d_{2} \frac{\partial^{2} \dot{v}}{\partial x^{2}}+r_{2}(x+c t) \dot{v}-\dot{v}^{2}-a_{2} \dot{u} \dot{v} .
\end{array}\right.
$$

It is easy to prove that $(\dot{u}(t, x), \dot{v}(t, x))$ with $(\dot{u}(0, x), \dot{v}(0, x))=\left(r_{1}(\infty), r_{2}(-\infty)\right)$ is an upper solution of the model (46). That means

$$
0 \leq u(t, x) \leq \dot{u}(t, x), \text { and } 0 \leq v(t, x) \leq \dot{u}(t, x)
$$

From Lemma 2.1 in Li et al. (2015), for any positive $\epsilon$, there exists $T>0$ and $M>0$, such that

$$
\begin{gathered}
\dot{u}(t, x)<\epsilon \text { for } t>T \text { and } x \leq-M+c t, \\
\dot{v}(t, x)<\epsilon \text { for } t>T \text { and } x \geq M-c t .
\end{gathered}
$$

Therefore,

$$
\begin{gathered}
u(t, x)<\epsilon \text { for } t>T \text { and } x \leq-M+c t, \\
v(t, x)<\epsilon \text { for } t>T \text { and } x \geq M-c t .
\end{gathered}
$$

The proof is complete.

This Lemma shows that since the compatible habitat for the respective species becomes increasingly disjoint, we can safely ignore the effect of $u$ when $x \rightarrow-\infty$ and the effect of $v$ when $x \rightarrow \infty$.

We have the following theorem regarding the nonpersistence of both species.

Theorem III.1 Assume that Hypothesis III.1 is satisfied, $c_{1}^{*}(\infty)<c$, and $c_{2}^{*}(-\infty)<$ c. Let $(u(t, x), v(t, x))$ be the solution of the system (46) with initial values $0 \leq$ $u(0, x) \leq r_{1}(\infty)$ and $0 \leq v(0, x) \leq r_{2}(-\infty)$, where $u(0, x)$ is zero for sufficiently large $x$ and $v(0, x)$ is zero for sufficiently negative $x$. For any positive $\epsilon$, there exists $T>0$ such that $u(t, x)<\epsilon$ and $v(t, x)<\epsilon$ for $t \geq T$ and $-\infty<x<\infty$. 
Remark III.1 With the assumption, as throughout this paper, that $r_{1}(-\infty)<0$, $r_{1}(\infty)>0$ and $r_{2}(-\infty)>0, r_{1}(\infty)<0$, we obtain the result summarized in theorem III.1. That is, if $c$ is large, then for any fixed point in space, after a sufficiently long time, both species disappear from that point.

From a biological point of view this is not at all surprising as the suitable habitat for $u$ drifts right, eventually leaving any point in space in unsuitable habitat for $u$. Similarly the suitable habitat for $v$ drifts left, eventually leaving any point in space in unsuitable habitat for $v$.

Proof. By the definition of $\phi_{i}(x ; \mu)$ with $i=1,2$, both decrease for $0<\mu<$ $\mu_{i}^{*}(x)$. Then for any $\epsilon>0$, there exist $\mu_{1}$ and $\mu_{2}$ such that $\phi_{1}\left(\infty ; \mu_{1}\right)=c_{1}^{*}(\infty)+\epsilon$ and $\phi_{2}\left(-\infty ; \mu_{2}\right)=c_{2}^{*}(-\infty)+\epsilon$. We assume $A_{1}$ and $A_{2}$ are positive numbers, and

$$
(\hat{u}(t, x), \hat{v}(t, x))=\left(A_{1} e^{-\mu_{1}\left(x-\left(c_{1}^{*}(\infty)+\epsilon\right) t\right)}, A_{2} e^{\mu_{2}\left(x+\left(c_{2}^{*}(-\infty)+\epsilon\right) t\right)}\right) .
$$

It is easy to prove that $(\hat{u}(t, x), \hat{v}(t, x))$ is the solution of

$$
\left\{\begin{array}{l}
\frac{\partial u}{\partial t}=d_{1} \frac{\partial^{2} u}{\partial x^{2}}+r_{1}(\infty) u \\
\frac{\partial v}{\partial t}=d_{2} \frac{\partial^{2} v}{\partial x^{2}}+r_{2}(-\infty) v .
\end{array}\right.
$$

Since

$$
r_{1}(\infty) u>r(x-c t) u-u^{2}-a_{1} u v
$$

and

$$
r_{2}(-\infty) v>r(x+c t) v-v^{2}-a_{2} u v
$$

we can show that $(\hat{u}(t, x), \hat{v}(t, x))$ is an upper solution of the model (46). Choose $A_{1}$ and $A_{2}$ sufficiently large such that $\hat{u}(0, x) \geq u(0, x)$ and $\hat{v}(0, x) \geq v(0, x)$. It follows that $0 \leq u(t, x) \leq \hat{u}(t, x)$ and $0 \leq v(t, x) \leq \hat{v}(t, x)$

Similar to the proof of statement (c) in Theorem II.1, and applying the Lemma III.1, we have that for every $\epsilon>0$, given $u(0, x)$ is zero for sufficiently large $x$ and $v(0, x)$ is zero for sufficiently negative $x$, there exists $T>0$ such that for $t \geq T$, $u(t, x)<\epsilon$ and $v(t, x)<\epsilon$ for all $x$.

The proof is complete. 


\section{Spreading Speed}

In the following Theorem III.2 we make the same assumptions on $r_{1}(x), r_{2}(x)$ as in theorem III.1. We also assume that the leftward spreading speed of $v$ is not sufficient to keep pace with the leftward shift of its suitable habitat boundary $\left(c_{2}^{*}(-\infty)<c\right)$. However, the spreading speed of $u$ is sufficiently fast that $u$ can keep ahead of the rightward shift of its suitable habitat boundary $\left(c<c_{1}^{*}(\infty)\right)$.

Theorem III.2 Assume that Hypothesis III.1 is satisfied and c satisfies $c_{2}^{*}(-\infty)<$ $c<c_{1}^{*}(\infty)$. Let $(u(t, x), v(t, x))$ be the solution of the system (46) with initial values $0 \leq u(0, x) \leq r_{1}(\infty)$ and $0 \leq v(0, x) \leq r_{2}(-\infty)$. Then we have the following statements:

(a) If $v(0, x)$ is zero for sufficiently negative $x$, then there exists $T>0$ such that for $t \geq T, v(t, x)<\epsilon$ for all $x$.

(b) If $u(0, x)$ is zero for sufficiently negative $x$, then for any $\epsilon>0$,

$$
\lim _{t \rightarrow+\infty}\left[\sup _{x \leq t(c-\epsilon)} u(t, x)\right]=0
$$

(c) If $u(0, x)$ is zero for all sufficiently large $x$, then for any positive $\epsilon$,

$$
\lim _{t \rightarrow+\infty}\left[\sup _{x \geq t\left(c^{*}(\infty)+\epsilon\right)} u(t, x)\right]=0
$$

(d) If $u(0, x)>0$ on a closed interval, and $u(0, x)$ is zero for all sufficiently large $x$, then for every $\epsilon$ with $0<\epsilon<\frac{\left(c^{*}(\infty)-c\right)}{2}$,

$$
\lim _{t \rightarrow+\infty}\left[\sup _{t(c+\epsilon) \leq x \leq t\left(c^{*}(\infty)-\epsilon\right)}\left|r_{1}(\infty)-u(t, x)\right|\right]=0
$$

Remark III.2 The biological interpretation of Theorem III.2 is as follows.

(i) The statement (a) shows that species $v$ faces extinction in every part of the spatial domain.

(ii) The statement (b) shows that the density of species u will approach zero for the region behind the rightward moving habitat boundary for species $u$. 
(iii) The statement (c) shows that the density of species $u$ will approach zero for the region sufficiently in front of the advancing population of $u$, which would be $x>c_{2}^{*}(\infty) t$.

(iv) The statement (d) shows that for the region of space between the suitable habitat boundary for $u$ and the leading edge of the advancing population of $u,(c t<x<$ $\left.c_{2}^{*}(\infty) t\right)$, the density of the species will approach the maximum carrying capacity for $u, r_{1}(\infty)$.

(v) Statement (b) and statement (d) show that the left wavefront of species u moves to the right at speed $c$; statement (c) and statement (d) show that, the species $u$ persists and spreads to the right at asymptotic spreading speed $c_{1}^{*}(\infty)$.

Proof. The proof of the statement (a) is similar to the proof of Theorem III.1, and is omitted. It follows that when $t \geq T, v(t, x)<\epsilon$ for all $x$. We assume $t_{0}=T$. When $t>t_{0}$, we have that $u\left(r_{1}(x-c t)-u-a_{1} v\right) \geq u\left(r_{1}(x-c t)-u-a_{1} \epsilon\right)$, so that the solution of model

$$
\frac{\partial u}{\partial t}=d_{1} \frac{\partial^{2} u}{\partial x^{2}}+\left(r_{1}(x-c t)-\epsilon\right) u-u^{2}
$$

$\grave{u}(t, x)$, is a lower solution of model

$$
\frac{\partial u}{\partial t}=d_{1} \frac{\partial^{2} u}{\partial x^{2}}+\left(r_{1}(x-c t)\right) u-u^{2}-a_{1} v
$$

Similarly, we can prove that the solution of model

$$
\frac{\partial u}{\partial t}=d_{1} \frac{\partial^{2} u}{\partial x^{2}}+r_{1}(x-c t) u-u^{2}
$$

$u(t, x)$, is an upper solution of system (49) with the same initial data.

Since $\epsilon$ can be arbitrarily small, the upper solution and the lower solution of (49) have similar results as Theorem 2.2 in Li et al. (2015).

The proof is complete.

Similarly, we have a theorem corresponding to Theorem III.2 when $c_{1}^{*}(\infty)<c$ and $c_{2}^{*}(-\infty)>c$. Then the species $u$ will eventually become extinct and the species $v$ 
will dominate the whole spatial domain and move to the left at the asymptotic speed $c_{2}^{*}(-\infty)$. We omit this theorem here.

In the next theorem, we describe the scenario where for both $u, v$ 's natural spreading speed are sufficient for them to keep pace with their respective suitable habitat boundaries.

Theorem III.3 Assume that Hypothesis III.1 is satisfied and $c$ satisfies that $c>0$, $c_{1}^{*}(\infty)>c$ and $c_{2}^{*}(-\infty)>c$. Let $(u(t, x), v(t, x))$ be the solution of the system $(46)$ with initial values $0 \leq u(0, x) \leq r_{1}(\infty)$ and $0 \leq v(0, x) \leq r_{2}(-\infty)$. Then we have the following statements:

(a) For any $\epsilon>0$, let $u(0, x)$ be zero for sufficiently negative $x$ and $v(0, x)$ be zero for sufficiently large $x$, then

$$
\begin{aligned}
& \lim _{t \rightarrow+\infty}\left[\sup _{x \leq t(c-\epsilon)} u(t, x)\right]=0, \\
& \lim _{t \rightarrow+\infty}\left[\sup _{x \geq-t(c-\epsilon)} v(t, x)\right]=0 .
\end{aligned}
$$

(b) For any positive $\epsilon$, let $u(0, x)$ be zero for all sufficiently large $x$ and $v(0, x)$ be zero for all sufficiently negative $x$. Then

$$
\begin{gathered}
\lim _{t \rightarrow+\infty}\left[\sup _{x \geq t\left(c_{1}^{*}(\infty)+\epsilon\right)} u(t, x)\right]=0, \\
\lim _{t \rightarrow+\infty}\left[\sup _{x \leq-t\left(c_{2}^{*}(-\infty)+\epsilon\right)} v(t, x)\right]=0 .
\end{gathered}
$$

(c) If $u(0, x)>0$ on a closed interval, and $u(0, x)$ is zero for all sufficiently large $x$, and if $v(0, x)>0$ on a closed interval, and $v(0, x)$ is zero for all sufficiently negative $x$, then for every $\epsilon$ with $0<\epsilon<\min \left\{\frac{\left(c_{1}^{*}(\infty)-c\right)}{2}, \frac{\left(c_{2}^{*}(-\infty)-c\right)}{2}\right\}$

$$
\begin{gathered}
\lim _{t \rightarrow+\infty}\left[\sup _{t(c+\epsilon) \leq x \leq t\left(c^{*}(\infty)-\epsilon\right)}\left|r_{1}(\infty)-u(t, x)\right|\right]=0, \\
\lim _{t \rightarrow+\infty}\left[\sup _{-t\left(c_{2}^{*}(\infty)-\epsilon\right) \leq x \leq-t(c+\epsilon)}\left|r_{2}(-\infty)-u(t, x)\right|\right]=0 .
\end{gathered}
$$

Remark III.3 The theorem has the following interpretations. 
(i) The statement (a) shows that species $u$ and $v$ will both become extinct outside of their respective suitable habitats.

(ii) The statement (b) shows that the species $u$ will only spread rightward into its suitable habitat at its asymptotic spreading speed $c_{1}^{*}(\infty)$; similarly the species $v$ will only spread leftward into its suitable habitat at its asymptotic spreading speed $c_{2}^{*}(-\infty)$.

(iii) The statement (c) shows that for the region of space between the suitable habitat boundary for $u$ and the leading edge of the advancing population for $u$, (ct $<$ $x<c_{2}^{*}(\infty) t$ ), the density of the species will approach the maximum carrying capacity for $u, r_{1}(\infty)$. Similarly for the region of space between the suitable habitat boundary for $v$ and the leading edge of the advancing population for $v$, $\left(-c_{2}^{*}(-\infty) t<x<-c t\right)$, the density of the species will approach the maximum carrying capacity for $v, r_{2}(-\infty)$.

Proof. Since the solution of the model $(49),(\dot{u}(t, x), \dot{v}(t, x))$, is the ordered upper solution of system (46), we have that

$$
u(t, x) \leq \dot{u}(t, x) \text { and } v(t, x) \leq \dot{v}(t, x)
$$

Since $(\dot{u}(t, x), \dot{v}(t, x))$ satisfies the Theorem 2.2 in Li et al. (2015), we have that for every $\epsilon>0$, if $\dot{u}(0, x)$ is zero for sufficiently negative $x, \dot{v}(0, x)$ is zero for sufficiently large $x$,

$$
\begin{aligned}
& \lim _{t \rightarrow+\infty}\left[\sup _{x \leq t(c-\epsilon)} \dot{u}(t, x)\right]=0, \\
& \lim _{t \rightarrow+\infty}\left[\sup _{x \geq-t(c-\epsilon)} \dot{v}(t, x)\right]=0 .
\end{aligned}
$$

When $\dot{u}(0, x)$ is zero for all sufficiently large $x, \dot{v}(0, x)$ is zero for all sufficiently negative $x$,

$$
\begin{gathered}
\lim _{t \rightarrow+\infty}\left[\sup _{x \geq t\left(c_{1}^{*}(\infty)+\epsilon\right)} \dot{u}(t, x)\right]=0, \\
\lim _{t \rightarrow+\infty}\left[\sup _{x \leq-t\left(c_{2}^{*}(-\infty)+\epsilon\right)} \dot{v}(t, x)\right]=0 .
\end{gathered}
$$

Therefore, we have the statement (a) and (b). 
We now consider the statement (c). From Lemma III.1, for any positive $\epsilon$, there exists $T>0$ and $M>0$, such that

$$
\begin{gathered}
u(t, x)<\epsilon \text { for } t>T \text { and } x \leq-M+c t, \\
v(t, x)<\epsilon \text { for } t>T \text { and } x \geq M-c t .
\end{gathered}
$$

That means when $t \geq T, v(t, x)$ can be extremely small when $x \geq M-c t$. Similar to the proof of statement (iii) of Theorem 2.2 in $\mathrm{Li}$ et al. (2015), we have that for the model

$$
\frac{\partial u}{\partial t}=d_{1} \frac{\partial^{2} u}{\partial x^{2}}+\left(r_{1}(x-c t)-\epsilon_{1}\right) u-u^{2}
$$

when $u(0, x)$ is zero for all sufficiently large $x$, then for every $\epsilon$ with $0<\epsilon<\frac{\left(c_{1}^{*}(\infty)-c\right)}{2}$,

$$
\lim _{t \rightarrow+\infty}\left[\sup _{t(c+\epsilon) \leq x \leq t\left(c^{*}(\infty)-\epsilon\right)}\left|r(\infty)-\epsilon_{1}-u(t, x)\right|\right]=0 .
$$

Since $\epsilon_{1}$ can be arbitrarily small, when $\epsilon_{1}$ converges to zero, we have that

$$
\lim _{t \rightarrow+\infty}\left[\sup _{t(c+\epsilon) \leq x \leq t\left(c^{*}(\infty)-\epsilon\right)}\left|r_{1}(\infty)-u(t, x)\right|\right]=0 .
$$

Similarly, we can prove that if $v(0, x)>0$ on a closed interval, and $v(0, x)$ is zero for all sufficiently negative $x$, then for every $\epsilon$ with $0<\epsilon \leq \frac{\left(c_{2}^{*}(-\infty)-c\right)}{2}$,

$$
\lim _{t \rightarrow+\infty}\left[\sup _{-t\left(c_{2}^{*}(\infty)-\epsilon\right) \leq x \leq-t(c+\epsilon)}\left|r_{2}(\infty)-u(t, x)\right|\right]=0 .
$$

The proof is complete.

\section{Discussion for Lotka-Volterra Competition Model Chapter}

In this chapter, we analyzed the simplest Lotka-Volterra competition model by assuming the respective suitable habitats of species, represented by $u$ and $v$, are drifting away from the established population. We illustrated that

- When the drift speed of the habitats for competing species exceeds the respective species' spreading speeds, both of them will become extinct.

- When one species' population expansion rate exceeds the habitat drift rate, this species will persist and spread in its suitable habitat. 
- When the drift speed for the habitats of both species is slower than their respective maximum population expansion rate, each of the species will survive and spread into their respective habitats.

This extends beyond the reaction-diffusion models in Chapter II to the multispecies systems and shows the impact of contracting habitat edges on the persistence and spread of both competing species.

The discussions in this chapter are only the rudimentary work for multi-species models. There are still various questions to be considered in future work. For example, our results in this chapter can be extended to analyze two populations with similar logistic dynamics that also interact with each other via a more general Lotka-Volterra competition model. We are interested in those species' persistence and spread in the presence of climate-driven habitat shift. The model is given by

$$
\left\{\begin{array}{l}
\frac{d u}{d t}=d_{1} \frac{\partial^{2} u}{\partial x^{2}}+u\left(r_{1}\left(x-c_{1} t\right)-u-a_{1} v\right) \\
\frac{d v}{d t}=d_{2} \frac{\partial^{2} u}{\partial x^{2}}+v\left(r_{2}\left(x-c_{2} t\right)-v-a_{2} u\right) .
\end{array}\right.
$$

Here $u=u(t, x)$, and $v=v(t, x)$ are densities of two competing populations. In this model, $r_{1}\left(x-c_{1} t\right)$ describes the intrinsic growth rates for the species $u$, which is assumed to be a bounded piecewise continuously differentiable function. The spatial domain can be divided into two parts for species $u$. If $r_{1}\left(x-c_{1} t\right)<0$, then the region is not suitable for the species $u$ 's growth and when $r_{1}\left(x-c_{1} t\right)>0$, then the region is suitable for $u$ to persist there. Similarly, $r_{2}\left(x-c_{2} t\right)$ describes the intrinsic growth rates for the species $v$, which is assumed to be a bounded piecewise continuously differentiable function. The spatial domain is divided into the region which is not suitable for species $v$ 's growth when $r_{2}\left(x-c_{2} t\right)<0$ and the region which is suitable for the species $v$ growth when $r_{2}\left(x-c_{2} t\right)>0$. The signs of $c_{1}$ and $c_{2}$ determine the direction of shift for the suitable habitat edge for each species. By varying the values of $c_{1}, c_{2}$, and definitions of the function $r_{1}(x)$ and $r_{2}(x)$ in model (50), we may model various scenarios of habitat shift. We will pick some more complicated scenarios and provide their numerical simulations in the next section (Figure 5 to Figure 10 ). 
6 Simulations for two species model

We present some numerical simulations to model (46) with intrinsic growth functions:

$$
\begin{aligned}
& r_{1}(x)= \begin{cases}r_{1}^{+} & \text {if } x<0 \\
r_{1}^{-} & \text {otherwise }\end{cases} \\
& r_{2}(x)= \begin{cases}r_{2}^{+} & \text {if } x<0 \\
r_{2}^{-} & \text {otherwise }\end{cases}
\end{aligned}
$$

where $r_{1}^{+}, r_{1}^{-}, r_{2}^{+}$and $r_{2}^{-}$can be any positive or negative numbers and the initial data

$$
\begin{gathered}
u(0, x)=\left\{\begin{aligned}
\sin (x-50) & \text { if } 50<x<50+\pi \\
0 & \text { otherwise, }
\end{aligned}\right. \\
v(0, x)=\left\{\begin{aligned}
\sin (x+50+\pi) & \text { if }-50-\pi<x<-50 \\
0 & \text { otherwise. }
\end{aligned}\right.
\end{gathered}
$$

The species $u$ was initially introduced on the interval $[50,50+\pi]$ and the species $v$ was initially introduced on the interval $[-50-\pi,-50]$. Assume that $d_{1}, d_{2}, a_{1}$ and $a_{2}$ are positive parameters and habitat shifting speed $c$ can be positive or negative.

In the discussion of the simulations for model (46), we assume that $r_{1}^{+}>$ $0>r_{1}^{-}$and $r_{2}^{+}<0<r_{2}^{-}$, so that the maximum spreading speed for species $u$ is $c_{1}^{*}(\infty)=2 \sqrt{d_{1} r_{1}^{+}}$and the maximum spreading speed for species $v$ is $c_{2}^{*}(-\infty)=$ $2 \sqrt{d_{2} r_{2}^{-}} \cdot r_{1}(x-c t)$ moves to the right at a speed $c>0$ and $r_{2}(x+c t)$ moves to the left at a speed $c>0$. Numerical simulations were conducted using MATLAB. In the color map, red corresponds to species $v$, while blue corresponds to $u$. For each species, dark color represents higher population density region, while light color represent low population density region.

Figure 2 corresponds with Theorem III.1, which states that both species become extinct if their maximum spreading speeds are exceeded by the climate change speed $c$. The Figure 2 shows the front view (Figure 2a) and vertical view (Figure 2b) of the numerical solution of model (46), assuming that $r_{1}^{+}=4, r_{2}^{-}=1, r_{1}^{-}=r_{2}^{+}=$ $-0.1, d_{1}=0.5, d_{2}=1, a_{1}=1, a_{2}=2$, so that $c_{1}^{*}(\infty)=2 \sqrt{2}=2.828 \ldots, c_{2}^{*}(\infty)=2$. 
We also assume that $r_{1}(x)$ moves toward the right with speed $c=3$ and $r_{2}(x)$ moves toward the left with speed $c=3$. So $c>c_{1}^{*}(\infty)$ and $c>c_{2}^{*}(-\infty)$. From Figure 2, we can see that both species eventually become extinct from the spatial domain. We see species $u$ persists longer than species $v$, which is consistent with its spreading speed 2.828 being closer to the climate change speed $c=3$.

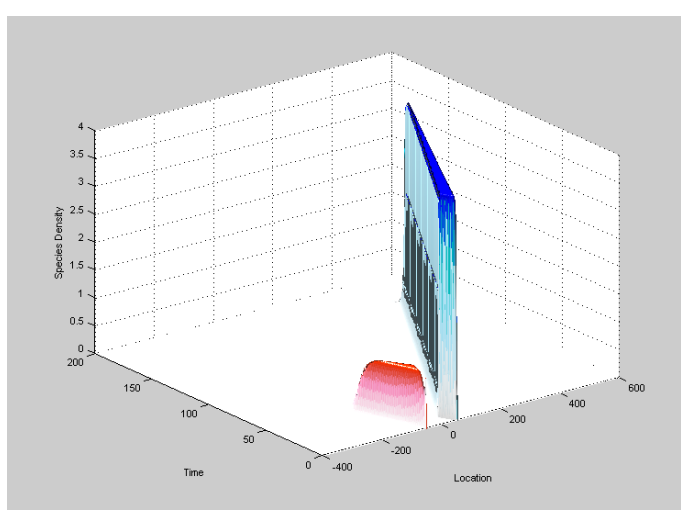

(a) Front View

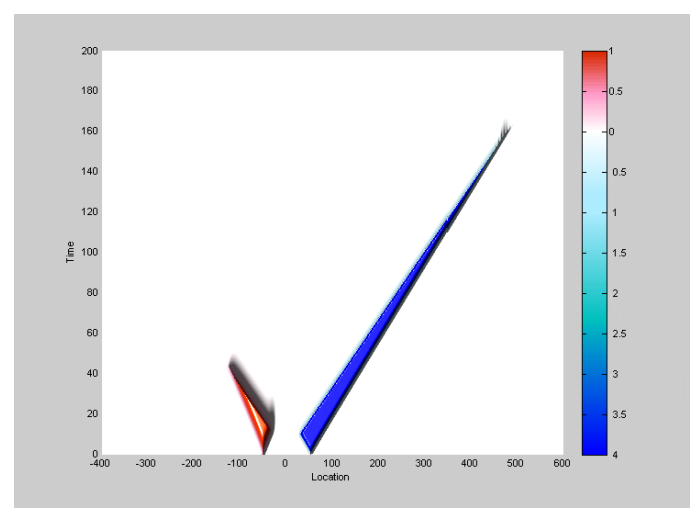

(b) Vertical View

Figure 2: Numerical Solution for Model (46) when $r_{1}^{+}=4, r_{2}^{-}=1, r_{1}^{-}=r_{2}^{+}=$ $-0.1, d_{1}=0.5, d_{2}=1, a_{1}=1, a_{2}=2$, and $c=3$

Figure 3 corresponds with Theorem III.2, which states that if $u$ 's maximum spreading speed exceeds $c$ while $v$ 's maximum spread speed is less than $c$, then $u$ will persist spreading at $c_{1}^{*}(\infty)$ and $v$ will face extinction. Figure 3 shows the front view (Figure 3a) and vertical view (Figure 3b) of the numerical solution of model (46), assuming that $r_{1}^{+}=4, r_{2}^{-}=1, r_{1}^{-}=r_{2}^{+}=-0.1, d_{1}=1.5, d_{2}=1, a_{1}=1, a_{2}=2$, so that $c_{1}^{*}(\infty)=2 * \sqrt{4 * 1.5}=4.898 \ldots, c_{2}^{*}(\infty)=2$. We also assume that $r_{1}(x-c t)$ moves toward the right with speed $c=2.2$ and $r_{2}(x+c t)$ moves toward the left with speed $c=2.2$. So $c_{1}^{*}(\infty)>c>c_{2}^{*}(-\infty)$. From Figure 3, we can see that the species $v$ eventually becomes extinct. The species $u$ moves to the right at an asymptotic speed $c_{1}^{*}(\infty)$. The rear of the wave shifts to the right at speed $c$.

Figure 4, which corresponds with Theorem III.3, shows the front view (Figure 4a) and vertical view (Figure 4b) of the numerical solution of model (46), assuming that $r_{1}^{+}=4, r_{2}^{-}=2, r_{1}^{-}=r_{2}^{+}=-0.1, d_{1}=1, d_{2}=1, a_{1}=1, a_{2}=2$, so that 


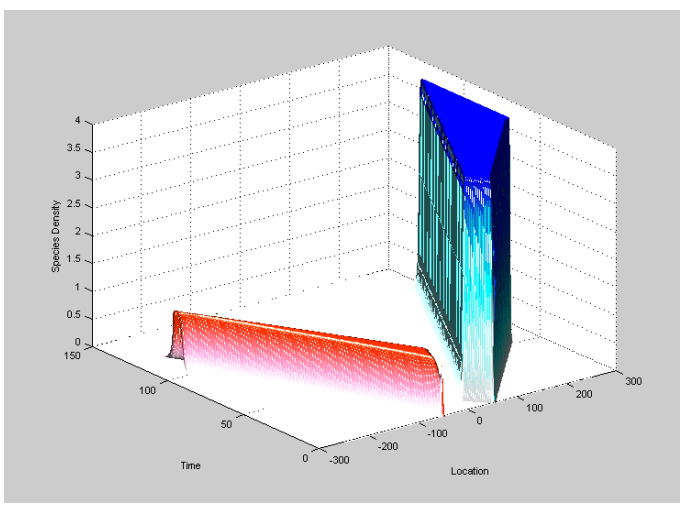

(a) Front View

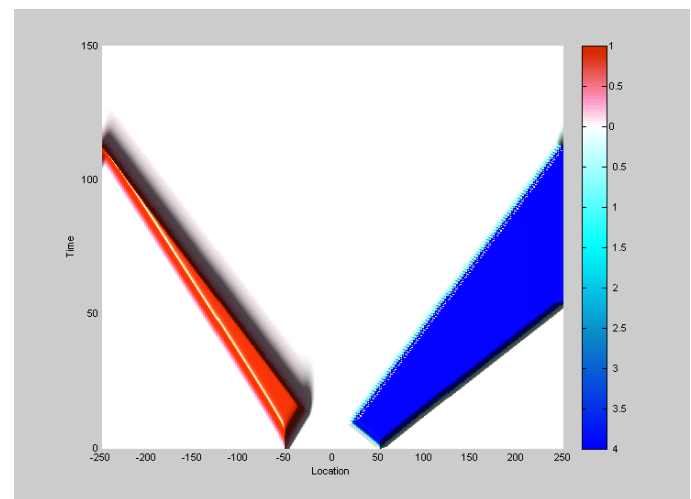

(b) Vertical View

Figure 3: Numerical Solution for Model (46) when $r_{1}^{+}=4, r_{2}^{-}=1, r_{1}^{-}=r_{2}^{+}=$ $-0.1, d_{1}=1.5, d_{2}=1, a_{1}=1, a_{2}=2$, and $c=2.2$

$c_{1}^{*}(\infty)=4, c_{2}^{*}(\infty)=2 \sqrt{2}=2.828 \ldots$ We also assume that both $r_{1}(x-c t)$ moves toward the right with speed $c=1.7$ and $r_{2}(x+c t)$ moves toward the left with speed $c=1$.7. So $c_{1}^{*}(\infty)>c_{2}^{*}(-\infty)>c$. From Figure 4 , we can see that species $u$ moves to the right at an asymptotic speed $c_{1}^{*}(\infty)$. Species $v$ moves to the left at an asymptotic speed $c_{2}^{*}(-\infty)$. The left wavefront of species $u$ moves to the right at speed $c$, similarly the right of the species $v$ wavefront moves to the left at speed $c$.

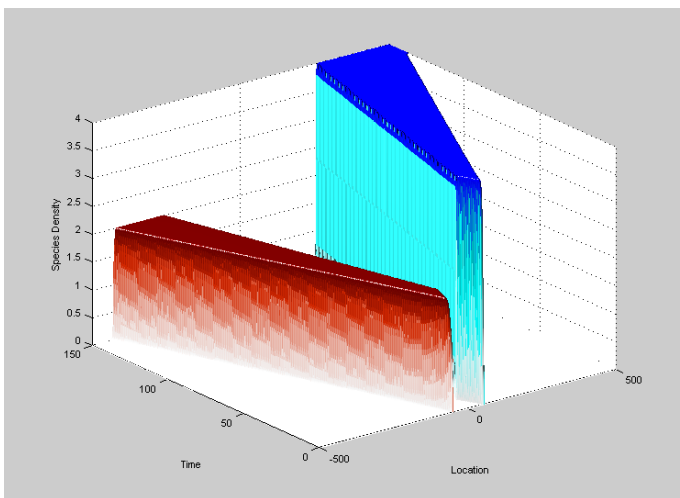

(a) Front View

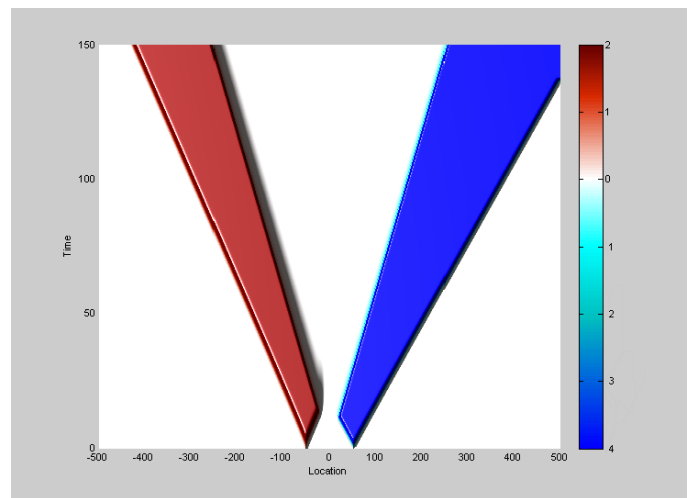

(b) Vertical View

Figure 4: Numerical Solution for Model (46) when $r_{1}^{+}=4, r_{2}^{-}=2, r_{1}^{-}=r_{2}^{+}=$ $-0.1, d_{1}=1, d_{2}=1, a_{1}=1, a_{2}=2$, and $c=1.7$

The competition model can be a difficult model to extract analytic results from, especially if exploring the persistence and spreading dynamics of both com- 
peting species along shifting habitats. Their spreading behavior will be affected by the resource distribution, habitat shifting speed, and competition coefficients of both species. In this chapter, we only analyzed the simplest situations, while there are many more interesting and complicated questions we were not able to include. We choose several of these more complicated scenarios and provide their numerical simulations here.

In these simulations, we model the following scenarios: To show the importance of competition coefficients, in Figures 5 and 6 we assume for both species the high quality habitat lies to the right, and the left most habitat is not suitable for survival. Further we assume both habitats drift rightward away from the established population, so that their suitable habitats are contracting. For purposes of contrast, we keep other parameters the same but vary the competition coefficients in Figures 5 and 6.

To show the importance of habitats shift directions, in Figures 7 and 8 we assume for both species the high quality habitat lies to the right, and the left most habitat is not suitable for survival. Further we assume both habitats drift leftward towards the established populations, so that their suitable habitats are expanding. For purposes of contrast with Figures 5 and 6, we keep other parameters the same but vary the habitat drift direction for both species in Figures 7 and 8 .

To show the importance of resource distribution for both species, in Figures 9 and 10 we assume for both species the high quality habitat lies to the right, and the left most habitat is also suitable for survival but of lower quality. Further we assume both habitats drift rightward away from the established populations. For purposes of contrast with Figures 5 and 6, we keep other parameters the same but vary the resource distribution for both species in Figures 9 and 10.

Figure 5 shows the front view (Figure 5a) and vertical view (Figure 5b) of the numerical solution of model (50), assuming that $r_{1}^{+}=1, r_{2}^{+}=2, r_{1}^{-}=r_{2}^{-}=$ $-0.1, d_{1}=1.5, d_{2}=2, a_{1}=0.1, a_{2}=1$, so that $c_{1}^{*}(\infty)=2 \sqrt{1.5}=2.449 \ldots, c_{2}^{*}(\infty)=4$. We also assume that both $r_{1}(x)$ and $r_{2}(x)$ move toward the right with speed $c=2$. So $c_{2}^{*}(\infty)>c_{1}^{*}(\infty)>c$. From Figure 5 , we can see the coexistence of species $u$ 


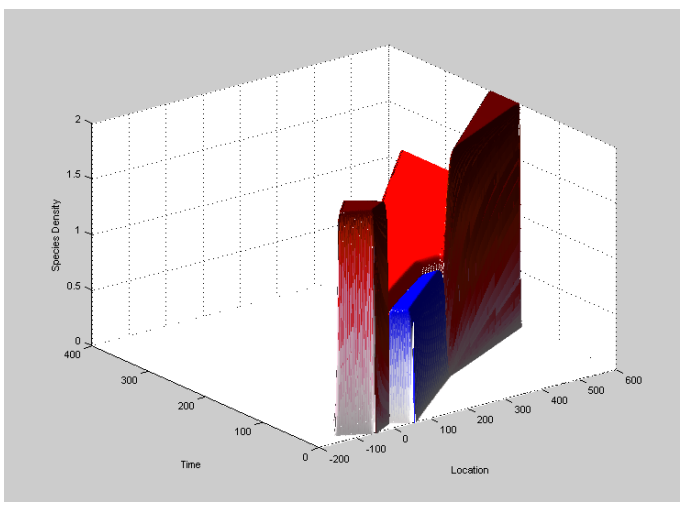

(a) Front View

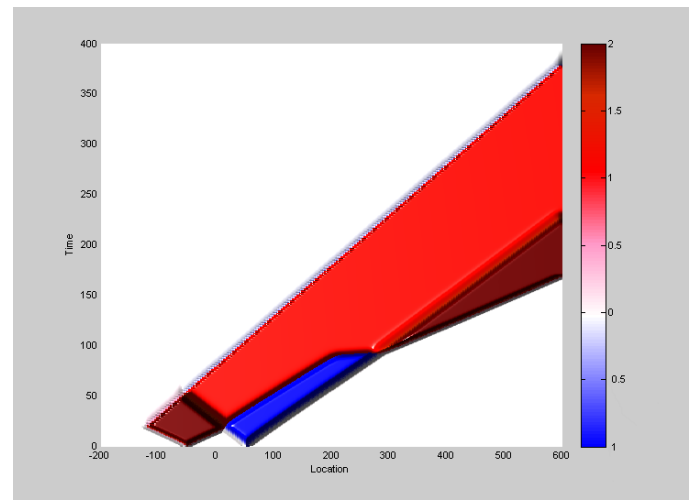

(b) Vertical View

Figure 5: Numerical Solution for Model (50) when $r_{1}^{+}=1, r_{2}^{+}=2, r_{1}^{-}=r_{2}^{-}=$ $-0.1, d_{1}=1.5, d_{2}=2, a_{1}=0.1, a_{2}=1$, and $c=2$

and species $v$ near the $\infty$ of the spatial domain. Species $v$ moves to the right at an asymptotic speed $c_{2}^{*}(\infty)$. The left wavefront of species $u$ and $v$ move to the right at speed $c$.

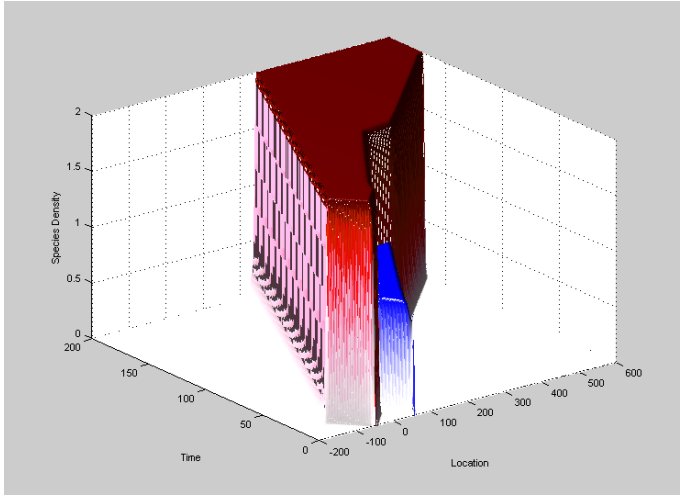

(a) Front View

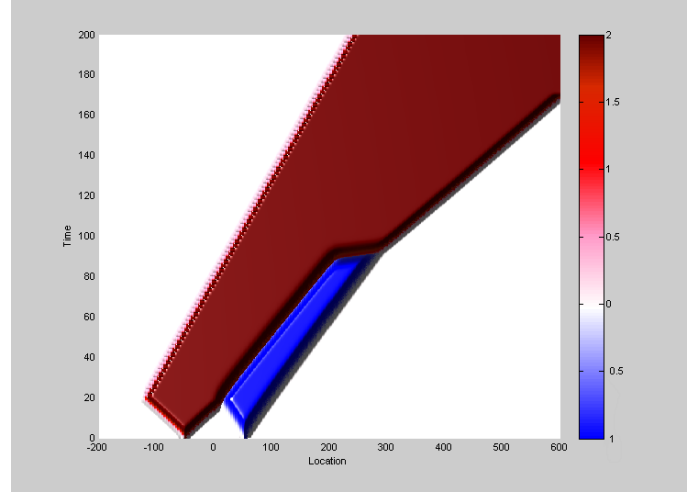

(b) Vertical View

Figure 6: Numerical Solution for Model (50) when $r_{1}^{+}=1, r_{2}^{+}=2, r_{1}^{-}=r_{2}^{-}=$ $-0.1, d_{1}=1.5, d_{2}=2, a_{1}=1, a_{2}=1$, and $c=2$

Figure 6 shows the front view (Figure 6a) and vertical view (Figure 6b) of the numerical solution of model (50), assuming that $r_{1}^{+}=1, r_{2}^{+}=2, r_{1}^{-}=r_{2}^{-}=$ $-0.1, d_{1}=1.5, d_{2}=2, a_{1}=1, a_{2}=1$, so that $c_{1}^{*}(\infty)=2 \sqrt{1.5}=2.449 \ldots, c_{2}^{*}(\infty)=4$. We also assume that both $r_{1}(x)$ and $r_{2}(x)$ move toward the right with speed $c=2$. So $c_{2}^{*}(\infty)>c_{1}^{*}(\infty)>c$. From Figure 6 , we can see that species $u$ becomes extinct 
and species $v$ moves to the right at an asymptotic speed $c_{2}^{*}(\infty)$. The left wavefront of species $v$ moves to the right at speed $c$.

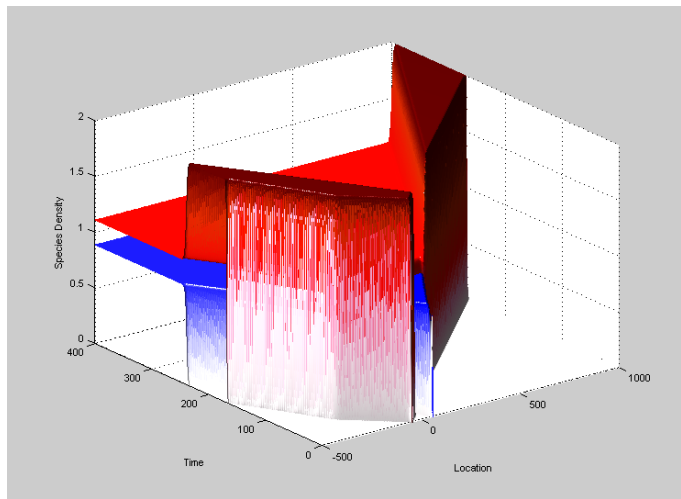

(a) Front View

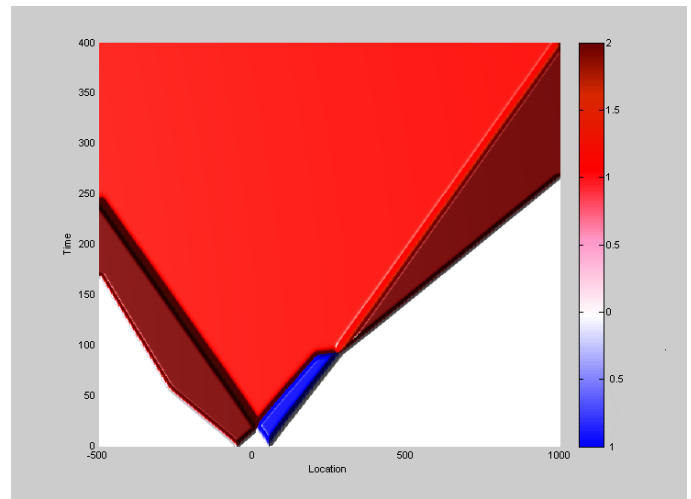

(b) Vertical View

Figure 7: Numerical Solution for Model (50) when $r_{1}^{+}=1, r_{2}^{+}=2, r_{1}^{-}=r_{2}^{-}=$ $-0.1, d_{1}=1.5, d_{2}=2, a_{1}=0.1, a_{2}=1$, and $c=-2$

Figure 7 shows the front view (Figure 7a) and vertical view (Figure 7b) of the numerical solution of model (50), assuming that $r_{1}^{+}=1, r_{2}^{+}=2, r_{1}^{-}=r_{2}^{-}=-0.1, d_{1}=$ $1.5, d_{2}=2, a_{1}=0.1, a_{2}=1$, so that $c_{1}^{*}(\infty)=2 * \sqrt{1.5}=2.449 \ldots, c_{2}^{*}(\infty)=4$. We also assume that both $r_{1}$ and $r_{2}$ move toward the left with speed $c=2$. So $c<c_{1}^{*}(\infty)$ and $c<c_{2}^{*}(-\infty)$. From Figure 7, we can see that species $u$ and species $v$ coexist everywhere in the spatial domain.

Figure 8 shows the front view (Figure 8a) and vertical view (Figure 8b) of the numerical solution of model (50), assuming that $r_{1}^{+}=1, r_{2}^{+}=2, r_{1}^{-}=r_{2}^{-}=$ $-0.1, d_{1}=1.5, d_{2}=2, a_{1}=1, a_{2}=1$, so that $c_{1}^{*}(\infty)=2 * \sqrt{1.5}=2.449 \ldots, c_{2}^{*}(\infty)=4$. We also assume that both $r_{1}(x)$ and $r_{2}(x)$ move toward the left with speed $c=2$. So $c<c_{1}^{*}(\infty)$ and $c<c_{2}^{*}(-\infty)$. From Figure 8 , we can see that species $u$ becomes extinct. Species $v$ moves to the right at an asymptotic speed $c_{2}^{*}(\infty)$ and moves to the left at speed $c$.

Figure 9 shows the front view (Figure 9a) and vertical view (Figure 9b) of the numerical solution of model (50), assuming that $r_{1}^{+}=1, r_{2}^{+}=2, r_{1}^{-}=0.8, r_{2}^{-}=$ $0.6, d_{1}=1.5, d_{2}=2, a_{1}=0.1, a_{2}=1$, so that $c_{1}^{*}(\infty)=2 * \sqrt{1.5}=2.449 \ldots, c_{2}^{*}(\infty)=$ $4, c_{1}^{*}(-\infty)=2 * \sqrt{0.8}=1.789 \ldots, c_{2}^{*}(-\infty)=2 * \sqrt{1.2}=2.191 \ldots$ We also assume that 


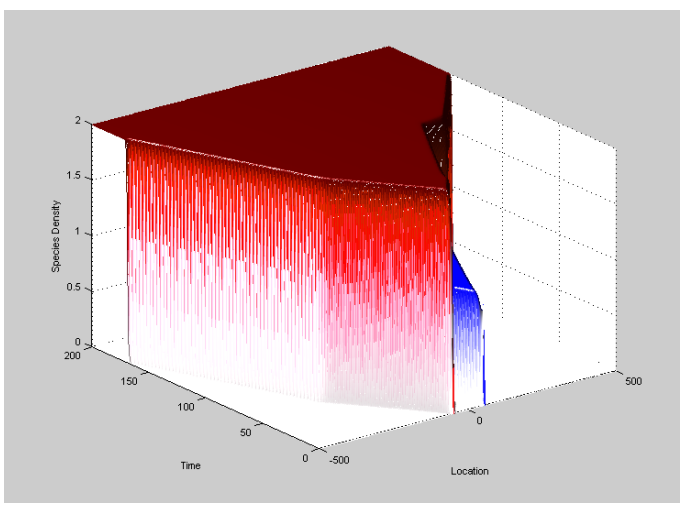

(a) Front View

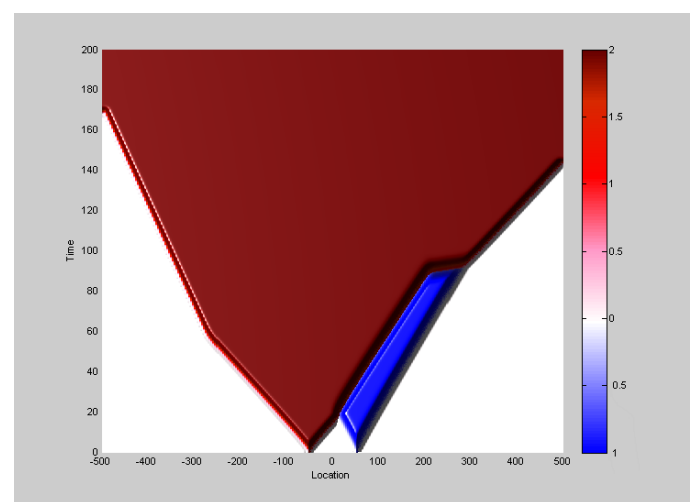

(b) Vertical View

Figure 8: Numerical Solution for Model (50) when $r_{1}^{+}=1, r_{2}^{+}=2, r_{1}^{-}=r_{2}^{-}=$ $-0.1, d_{1}=1.5, d_{2}=2, a_{1}=1, a_{2}=1$, and $c=-2$

both $r_{1}(x)$ and $r_{2}(x)$ move toward the right with speed $c=2$. So $c<c_{1}^{*}(\infty)$ and $c<c_{2}^{*}(-\infty)$. From Figure 9, we can see that the species $u$ and species $v$ coexist and compete with each other everywhere in the spatial domain. Species $v$ moves faster than species $u$ in both directions. The spreading speed of species $v$ in the right direction is $c_{2}^{*}(\infty)$ and in the left direction is $c_{2}^{*}(-\infty)$.

Figure 10 shows the front view (Figure 10a) and vertical view (Figure 10b) of the numerical solution of model (50), assuming that $r_{1}^{+}=1, r_{2}^{+}=2, r_{1}^{-}=0.8, r_{2}^{-}=$ $0.6, d_{1}=1.5, d_{2}=2, a_{1}=1, a_{2}=1$, so that $c_{1}^{*}(\infty)=2 * \sqrt{1.5}=2.499 . ., c_{2}^{*}(\infty)=4$. We also assume that both $r_{1}(x)$ and $r_{2}(x)$ move toward the right with speed $c=2$. So $c<c_{1}^{*}(\infty)$ and $c<c_{2}^{*}(-\infty)$. From Figure 10, we can see that species $u$ becomes extinct from the spatial domain. Species $v$ moves to the right at an asymptotic speed $c_{2}^{*}(\infty)=4$ and to the left at speed $c_{2}^{*}(-\infty)$. 


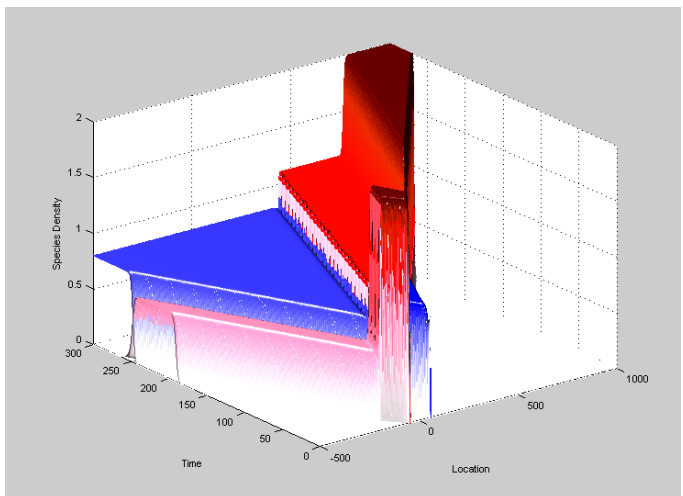

(a) Front View

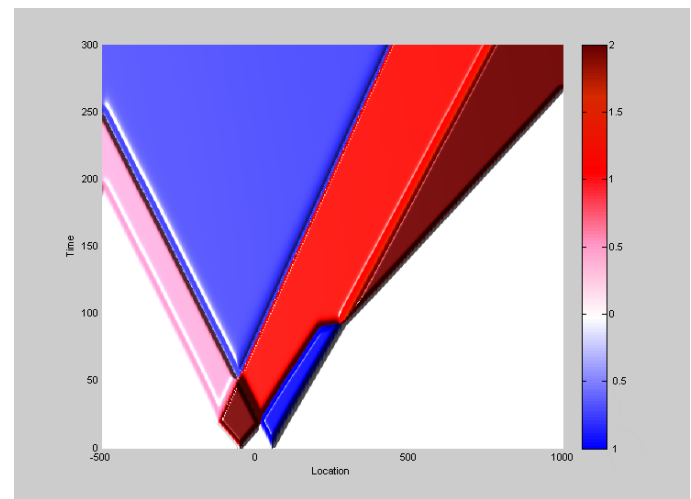

(b) Vertical View

Figure 9: Numerical Solution for Model (50) when $r_{1}^{+}=1, r_{2}^{+}=2, r_{1}^{-}=0.8, r_{2}^{-}=$ $0.6, d_{1}=1.5, d_{2}=2, a_{1}=0.1, a_{2}=1$, and $c=2$

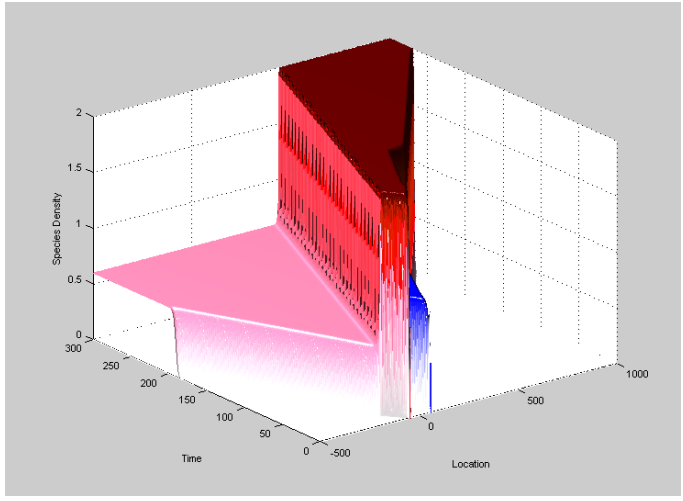

(a) Front View

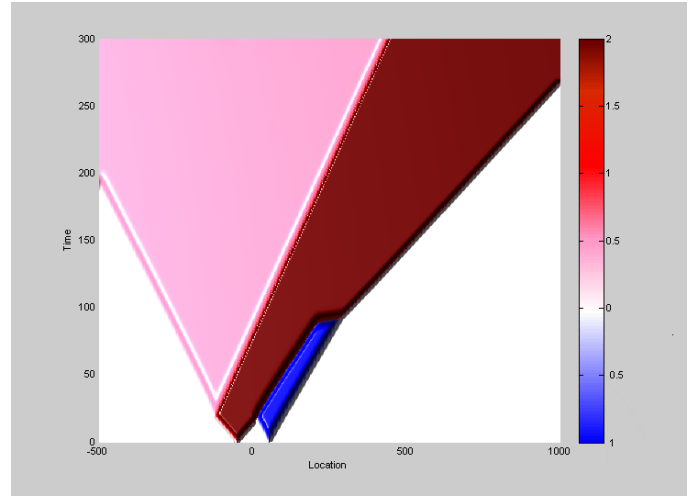

(b) Vertical View

Figure 10: Numerical Solution for Model (50) when $r_{1}^{+}=1, r_{2}^{+}=2, r_{1}^{-}=0.8, r_{2}^{-}=$ $0.6, d_{1}=1.5, d_{2}=2, a_{1}=1, a_{2}=1$, and $c=2$ 


\section{CHAPTER IV}

\section{LATTICE DIFFERENTIAL MODEL ANALYSIS}

\section{Linear Lattice Differential Equations and Modified Bessel Functions}

In this chapter we are concerned about the spread and persistence of some species described by lattice differential equations. Lattice differential equations are infinite systems of ordinary differential equations indexed by points on a spatial lattice. Such systems have emerged in many applications, for example, in physics(Scott 2003), chemistry(Laplante and Erneux 1992), biology(Bell 1981, Winslow et al. 1993, Aronson and Huang 1994) and material sciences(Cahn 1960, and Cook et al., 1969). They are also found to be useful in the theory of bio-macromolecules, for example, they have been successfully applied to describe the processes of the transformations and binding in gene regulation and signal transduction (Zhang 2003, Teif 2010).

In our work, we are mostly interested in their applications in mathematical population models, where the lattice can be regarded as patchy environments for some species. We will use the lattice differential equations to characterize some biological phenomena, like spread and persistence for some species. We like to mention that there has been a large volume of work devoted to the study of existence of traveling wave solutions to the lattice differential equations with various growth functions and functionals (Chen and Guo 2002, Cahn 1998, Carr 2004, Chi et al. 1986, Chow et al. 1998, Fang et al. 2010, Iooss 2000, Keener 1987, Ma and Zou 2005, Mallet-Paret 1999, Wu and Zou 1997, Zhang and Guo 2014, Zinner 1992, Zinner et al. 1992), however to the systems under our interest, the existence of traveling wave solutions remains to be discussed due to the inhomogeneity of the nonlinear terms. 
We begin with the linear lattice differential equations:

$$
\left\{\begin{array}{l}
\frac{\partial u}{\partial t}(t, x)=D[u(t, x+1)-2 u(t, x)+u(t, x-1)], \quad x \in \mathrm{H}, t>0, \\
u(0, x)=u_{0}(x),
\end{array}\right.
$$

where the initial data $u_{0}$ is assumed to be in $\mathrm{L}^{\infty}(\mathrm{H})$. Here $\mathrm{H}$ is the habitat which can be the discrete integer space $\mathrm{Z}$ or the continuous space $\mathrm{R}$. In this model, $u(t, x)$ represents the population density of the species at point $x$ time $t$. The coefficient $D$ represents the diffusivity whose counterpart can be found in the classical heat equations $\frac{\partial u}{\partial t}=D \frac{\partial^{2} u}{\partial x^{2}}$. The system of (51) can be viewed as approximation of the heat equation by discretization in the spatial direction.

To proceed, we introduce some notations. Let $\Delta_{1}$ and $\Delta_{-1}$ be the shifting operators as $\left(\Delta_{1} u\right)(x)=u(x+1)$ and $\left(\Delta_{-1} u\right)(x)=u(x-1)$ for any $u \in \mathrm{L}^{\infty}(\mathrm{H})$, and I be the identity operator in $\mathrm{L}^{\infty}(\mathrm{H})$. It is trivial to notice that $\Delta_{1} \cdot \Delta_{-1}=\Delta_{-1} \cdot \Delta_{1}=\mathrm{I}$. The operator $\Delta_{1}-2 \mathrm{I}+\Delta_{-1}$ is the discretized Laplace operator. With these notations, the linear lattice differential equations (51) can be written as an abstract ODE system in $L^{\infty}(\mathrm{H})$,

$$
\frac{\partial u}{\partial t}=D\left(\Delta_{1}-2 \mathrm{I}+\Delta_{-1}\right) u
$$

Following the work in $\mathrm{Hu}$ and $\mathrm{Li}$ (2015) concerning the fundamental solution theory to the linear lattice differential model (51), the solution $u(t, x)$ can be given by

$$
\begin{aligned}
u(t, x) & =\left[e^{D\left(\Delta_{1}-2 \mathrm{I}+\Delta_{-1}\right) t} u_{0}\right](x) \\
& =e^{-2 D t} \sum_{m \in Z} I_{m}(2 D t) u_{0}(x-m),
\end{aligned}
$$

where $I_{m}, m \geq 0$ are defined as

$$
I_{m}(t)=\sum_{k=0}^{+\infty} \frac{(t / 2)^{m+2 k}}{k !(m+k) !}
$$

and $I_{m}(t)=I_{-m}(t)$ for $m<0$. The sequence $\left\{I_{m}(t)\right\}_{m \geq 0}$ turns out to be the classical first kind modified Bessel function of order $m$. As far as we know, Hu and Li are the first to make the connection between the modified Bessel functions and fundamental solutions to lattice differential equations.

As $u(t, x) \equiv$ constant is a solution of model $(51)$, it is easy to prove that

$$
1=e^{-2 D t}\left[I_{0}(2 D t)+2 I_{1}(2 D t)+2 I_{2}(2 D t)+2 I_{3}(2 D t)+\cdots\right] .
$$


Here we borrow some important estimates on $I_{m}(t)$ obtained in $\mathrm{Hu}$ and $\mathrm{Li}$ (2015) for our analysis. The first one is Lemma 2.1 in $\mathrm{Hu}$ and $\mathrm{Li}$ (2015).

Lemma IV.1 For $m \leq 2 D t$

$$
\begin{gathered}
e^{-2 D t} I_{m}(2 D t) \leq \frac{C}{\sqrt{2 \pi}} \frac{e^{-\alpha_{1} \frac{m^{2}}{2 D t}}}{\left((2 D t)^{2}+m^{2}\right)^{1 / 4}}, \\
e^{-2 D t} I_{m}^{\prime}(2 D t) \leq \frac{C}{\sqrt{2 \pi}} \frac{\left((2 D t)^{2}+m^{2}\right)^{1 / 4}}{2 D t} e^{-\alpha_{1} \frac{m^{2}}{2 D t}}
\end{gathered}
$$

For $m \geq 2 D t$

$$
\begin{gathered}
e^{-2 D t} I_{m}(2 D t) \leq \frac{C}{\sqrt{2 \pi}} \frac{e^{-\alpha_{2} m}}{\left((2 D t)^{2}+m^{2}\right)^{1 / 4}}, \\
e^{-2 D t} I_{m}^{\prime}(2 D t) \leq \frac{C}{\sqrt{2 \pi}} \frac{\left((2 D t)^{2}+m^{2}\right)^{1 / 4}}{2 D t} e^{-\alpha_{2} m},
\end{gathered}
$$

where $\alpha_{1}=\ln (\sqrt{2}+1)-\frac{1}{2}$ and $\alpha_{2}=\ln (\sqrt{2}+1)+1-\sqrt{2}$. C is an absolute constant which may vary at each occurrence.

We comment that the estimate (53) shows that the kernel $e^{-2 D t} I_{m}(2 D t)$ behaves like the Gaussian kernel when $m$ is small and the estimate (54) shows that the kernel $e^{-2 D t} I_{m}(2 D t)$ behaves like the exponentially decaying when $m$ is large as the time $t$ is fixed. We have the following corollary for the tail estimates on $u(t, x)$ (Corollary 2.2 in $\mathrm{Hu}$ and $\mathrm{Li}(2015)$ ).

Corollary IV.1 For any positive $\epsilon \in(0,1)$, there exists an $M>1$ depending only on $\epsilon$, such that,

$$
\sum_{|m| \geq \max \{M, 2 D t\}} e^{-2 D t} I_{m}(2 D t) \leq \frac{\epsilon}{2},
$$

and

$$
\sum_{|m| \geq \max \{M, \sqrt{2 D M t}\}} e^{-2 D t} I_{m}(2 D t) \leq \epsilon
$$


By taking the resource limitations into consideration, we propose to study the simplest nonlinear lattice differential equation model:

$$
\frac{\partial u}{\partial t}=D[u(t, x+1)-2 u(t, x)+u(t, x-1)]+u(r-u), \quad x \in \mathrm{H}, \quad t>0 .
$$

The constant $r$ represents the capacity of the environment. This model was also applied to nerve systems (Bell 1981). The spreading speed of the traveling wave solutions connecting 0 and $r$ of model (55) is obtained as $c^{*}=\inf _{\mu>0} \frac{4 D \sinh ^{2}(\mu / 2)+r}{\mu}$ (Fang 2010, Liang 2007 and Weng 2003). The equation (55) is also an extension of the famous Fisher-KPP equation for spatial population ecology in discrete space. Additionally, with different growth functions and functionals, various extensions of (55) have been proposed and investigated extensively in recent years (Chen 2002, Cahn 1998, Carr 2004, Chi 1986). We conclude this section with the nonhomogeneous system defined as follows

$$
\left\{\begin{array}{l}
\frac{\partial u}{\partial t}(t, x)=D[u(t, x+1)-2 u(t, x)+u(t, x-1)]+f(t, x), x \in \mathrm{H}, t>0 \\
u(0, x)=u_{0}(x)
\end{array}\right.
$$

where the initial data $u_{0}$ is assumed to be in $\mathrm{L}^{\infty}(\mathrm{H})$ and $f \in \mathrm{L}^{\infty}\left(\mathrm{R}^{+}, \mathrm{H}\right)$. With aid of fundamental solutions of the linear lattice differential equation (51), the solution of model $(56) u(t, x)$ can be written as follows

$$
\begin{aligned}
u(t, x)= & \sum_{m=-\infty}^{+\infty} e^{-2 D t} I_{m}(2 D t) u_{0}(x-m) \\
& +\int_{0}^{t} \sum_{m=-\infty}^{+\infty} e^{-2 D(t-\tau)} I_{m}(2 D(t-\tau)) f(\tau, x-m) d \tau .
\end{aligned}
$$

2 Well-posedness of Lattice-Differential Model with Shifting Habitat

In this section we are concerned about the growth and spread of a species described by the lattice differential equations with shifting habitat as follows:

$$
\frac{\partial u}{\partial t}(t, x)=D[u(t, x+1)-2 u(t, x)+u(t, x-1)]+r(x-c t) u(t, x)-u^{2}(t, x), \quad x \in \mathrm{H}, t>0 .
$$

Here $u(t, x)$ is the density function for the population at point $\mathrm{x}$ and time $\mathrm{t}, D>0$ is a constant diffusion coefficient, $r(x)$ represents the population growth rate, $-u^{2}$ 
describes the death rate due to the resource limitation, and $\mathrm{H}$ is the habitat which can be the discrete integer space $\mathrm{Z}$ or the continuous space $\mathrm{R}$.

We assume that $r(x)$ is continuous and nondecreasing and bounded from below by $r(-\infty)$ and above by $r(\infty)>0$. The monotonicity of $r(x)$ in $x$ reflects that the quality of habitat improves to the right along the $x$-axis. The assumption $r(x) \leq$ $r(\infty)$ indicates that the population growth rate is limited by the maximum carrying capacity of the environment. We use the function $r(x-c t)$ to represent the habitat shifting with climate change, where $c$ is a real number. When $c$ is positive, the function $r(x-c t)$ implies that the resource distribution propagates rightward at speed $c$, so that the habitat with better quality contracts. When $c$ is negative, this function implies that the resource distribution propagates leftward at speed $|c|$, so that the habitat with better quality expands. The persistence and spread of species in a discrete space with shifting habitat have been studied by assuming $r(-\infty)<0$ (Hu and Li (2015)), which means, to the left of the region of poor quality, the species cannot grow. In this Chapter, we assume that $r(-\infty)>0$, then the species can grow everywhere along the discrete spatial gradient, but with differing levels of success. The potential domain of the species is unbounded and can be distinguishable as a higher quality region with higher population growth rate (favorable habitat) and a lower quality region with lower population growth rate (less favorable habitat). The edge of the favorable habitat for species is shifting at speed $c$. We are interested in the spread and persistence of the species for different $c$ values.

We make the following hypothesis for $r(x)$ for our mathematical analysis:

Hypothesis IV.1 $r(x)$ is nondecreasing, bounded, and piecewise continuously differentiable in $x$ for $-\infty<x<\infty, 0<r(-\infty)<r(\infty)<\infty$.

With regard to problem (58), we have the following definition of upper and lower solutions.

Definition IV.1 A function $\tilde{u}(t, x)$ with $t>0$ and $x \in \mathrm{R}$ is called an upper solution of (58) if the following inequality is satisfied:

$$
\frac{\partial \tilde{u}}{\partial t} \geq D[\tilde{u}(t, x+1)-2 \tilde{u}(t, x)+\tilde{u}(t, x-1)]+\tilde{u} r(x-c t)-\tilde{u}^{2}, \quad x \in \mathrm{H}, t>0 .
$$


Similarly, $\hat{u}(t, x)$ is called a lower solution if the reversed inequality in (59) is satisfied.

The functions $\tilde{u}(t, x)$ and $\hat{u}(t, x)$ are called ordered upper and lower solutions if $\tilde{u}(t, x) \geq \hat{u}(t, x)$ for all $t>0$ and $-\infty<x<\infty$. From the definition, it is clear that $\tilde{u}(t, x)=r(\infty)$ is an upper solution and $\hat{u}(t, x)=0$ is a lower solution of model (58). To study the existence of a solution to (58), we need some conditions on the population growth function. A basic assumption on $f(u, t, x)=u r(x-c t)-u^{2}$ is the following one-sided Lipschitz condition:

$$
\begin{aligned}
f\left(u_{1}, t, x\right)-f\left(u_{2}, t, x\right) & =u_{1} r(x-c t)-u_{1}^{2}-\left(u_{2} r(x-c t)-u_{2}^{2}\right) \\
& =\left(u_{1}-u_{2}\right)\left(r(x-c t)-u_{1}-u_{2}\right) \\
& \geq-\rho\left(u_{1}-u_{2}\right),
\end{aligned}
$$

for $0 \leq u_{2} \leq u_{1} \leq r(\infty)$ and $\rho$ is a constant. It is easy to prove that $u r(x-c t)-u^{2}$ is Lipschitz continuous with $\rho>3 r(\infty)$, then the function

$$
\mathrm{F}(u, t, x) \equiv u(\rho+r(x-c t)-u)
$$

is nondecreasing in $u$ for $0 \leq u \leq r(\infty)$. By adding a dominant linear term $\rho u(t, x)$ to both sides of model (58), we obtain the equivalent equation of (58):

$$
\frac{\partial u}{\partial t}+\rho u=D[u(t, x+1)-2 u(t, x)+u(t, x-1)]+\rho u+u r(x-c t)-u^{2} .
$$

Obviously, $u \equiv 0$ is a trivial solution and $u \equiv r(\infty)$ is an upper solution of (60). From $\mathrm{Hu}$ and $\mathrm{Li}(2015)$, the solution of $(60)$ with $u(0, x)=u_{0}(x)$, where $u_{0}(x)$ is continuous in $x$ and $0 \leq u_{0}(x) \leq r(\infty)$, can be expressed as the fixed point of the nonlinear integral equation as follows,

$$
\begin{aligned}
u(t, x)= & (T[u])(t, x) \\
= & \sum_{m=-\infty}^{+\infty} e^{-(\rho+2 D) t} I_{m}(2 D t) u_{0}(x+m) \\
& +\int_{0}^{t} \sum_{m=-\infty}^{+\infty} e^{-(\rho+2 D)(t-\tau)} I_{m}(2 D(t-\tau)) \\
& \cdot u(\tau, x+m)[\rho+r(x+m-c \tau)-u(\tau, x+m)] d \tau,
\end{aligned}
$$

with $\rho>3 r(\infty)$. 
Consider the sequence $u^{(n)}(t, x)$ generated by:

$$
u^{(n+1)}(t, x)=\left(T\left[u^{(n)}\right]\right)(t, x),
$$

where $u^{(0)}(t, x)=0$ or $u^{(0)}(t, x)=r(\infty)$. Theorem 3.1 in (Hu and Li, 2015) claims that the sequence $u^{(n)}(t, x)$ is nondecreasing in $n$ if $u^{(0)}(t, x)=r(-\infty)$ and nonincreasing in $n$ if $u^{(0)}(t, x)=r(\infty)$. In both cases $u(t, x)=\lim _{n \rightarrow \infty} u^{(n)}(t, x)$ is the unique solution of model $(60)$ with $u(0, x)=u_{0}(x)$, and $0 \leq u(t, x) \leq r(\infty)$.

Let

$$
\mathrm{L}_{\alpha}:=\{u: 0 \leq u(x) \leq \alpha \text { for } x \in \mathrm{H}\} \subseteq \mathrm{L}^{\infty}(\mathrm{H})
$$

We present below the comparison principle corresponding to the lattice differential equation (ref. Corollary 3.1 in $\mathrm{Hu}$ and $\mathrm{Li}$ (2015)), which will be the principal tool in subsequent discussions.

Lemma IV.2 (Comparison Principle) Let $u, v \in C\left(R^{+}, \mathrm{L}_{r(\infty)}\right)$, such that $u(t, x) \geq$ $(T(u))(t, x)$ and $v(t, x) \leq(T(u))(t, x)$ for all $(t, x) \in R^{+} \times \mathrm{H}$ and $u(0, x) \geq v(0, x)$ for all $x \in \mathrm{H}$. Then $u(t, x) \geq v(t, x)$ for all $(t, x) \in R^{+} \times \mathrm{H}$.

To consider the spreading speed of the lattice differential equation, we introduce some notations. Define

$$
\phi(x ; \mu)=\frac{4 D \sinh ^{2}(\mu / 2)+r(x)}{\mu},
$$

and

$$
\psi(\mu)=D\left(e^{\mu}-e^{-\mu}\right)
$$

Then following $\mathrm{Hu}$ and $\mathrm{Li}$ (2015), we define the spreading speed

$$
c^{*}(x)=\inf _{\mu>0} \phi(x ; \mu)
$$

which is the unique minimum point of $\phi(x ; \mu)$. Let $\mu=\mu^{*}(x)$ be the unique point where the minimum of $\phi(x ; \mu)$ occurs, then

$$
c^{*}(x)=\phi\left(x ; \mu^{*}(x)\right)
$$

and by direct calculation, $\mu=\mu^{*}(x)$ is increasing in $\mathrm{x}$. 
Observe that $\psi(\mu)$ is a strictly increasing function for $\mu>0$ and $\phi(x ; \mu)$ is strictly decreasing for $0 \leq \mu \leq \mu^{*}(x)$ at each number $x$. We have that $\phi(x ; \mu)>\psi(\mu)$ for $0<\mu<\mu^{*}(x)$. Furthermore, $\phi(x ; \mu)$ intersects with $\psi(\mu)$ at the point where the infimum of $\phi(x ; \mu)$ is attained, i.e.,

$$
c^{*}(x)=\phi\left(x ; \mu^{*}(x)\right)=\psi\left(\mu^{*}(x)\right) .
$$

It is easy to prove that when $r(\infty)>r(-\infty)>0, c^{*}(\infty)>c^{*}(-\infty)$. We will demonstrate that under appropriate conditions, the rightward spreading speed is given by $c^{*}(\infty)$ or $c^{*}(-\infty)$, and the leftward spreading speed is given by $c^{*}(\infty)$, $c^{*}(-\infty)$ or $|c|$.

\section{Upper Solutions}

In this section we discuss the upper solutions for model (58). When $c=0$, we provide a lemma for the equation,

$$
\frac{\partial u}{\partial t}=D[u(t, x+1)-2 u(t, x)+u(t, x-1)]+r(x) u-u^{2}, \quad x \in \mathrm{H}, t>0,
$$

which is essentially Lemma 4.1 in $\mathrm{Hu}$ and $\mathrm{Li}$ (2015).

Lemma IV.3 Let $\bar{u}(t, x)$ be the solution of model (64) with $\bar{u}(0, x)=r(\infty)$. Then $\bar{u}(t, x)$ is non-increasing in $t$ and nondecreasing in $x, \bar{u}(t,-\infty)=r(-\infty)$, and $\bar{u}(t, \infty)=r(\infty)$ for $t>0$.

The proof of this lemma is similar to the one of Lemma 4.1 in Hu and $\mathrm{Li}$ (2015), so we omit the details.

Lemma IV.4 Let $u(t, x)$ be the solution of model (58) with $u(0, x)=u_{0}(x)$, where $0 \leq u_{0}(x) \leq r(\infty)$. For any $\epsilon>0$, there exists sufficiently large $T>0$ and $M>0$, such that, when $t>T$ and $x \leq-M+c t$,

$$
u(t, x) \leq r(-\infty)+\epsilon
$$

Proof. We first consider the case of $c \geq 0$. For any $\epsilon>0$, we take

$$
\epsilon_{0}=\left(\sqrt{(4+r(-\infty))^{2}+4 \epsilon}-4-r(-\infty)\right) / 2>0
$$


Let $w(t, x)=u(t, x)-r(-\infty)-\epsilon_{0}$. Since $u(t, x)$ is the solution of model (58) with $u(0, x)=u_{0}(x), w(t, x)$ satisfies the following equation:

$$
\begin{aligned}
\frac{\partial w}{\partial t}= & D[u(t, x+1)-2 u(t, x)+u(t, x-1)] \\
& +\left(w+r(-\infty)+\epsilon_{0}\right)\left(r(x-c t)-w-r(-\infty)-\epsilon_{0}\right),
\end{aligned}
$$

which is equivalent to:

$$
\begin{aligned}
\frac{\partial w}{\partial t}+\rho w= & D[u(t, x+1)-2 u(t, x)+u(t, x-1)] \\
& +\rho w+\left(w+r(-\infty)+\epsilon_{0}\right)\left(r(x-c t)-w-r(-\infty)-\epsilon_{0}\right) \\
= & D[u(t, x+1)-2 u(t, x)+u(t, x-1)] \\
& +\left(w+r(-\infty)+\epsilon_{0}\right)\left(\rho+r(x-c t)-w-r(-\infty)-\epsilon_{0}\right)-\rho(r(-\infty)+\epsilon),
\end{aligned}
$$

with $\rho>3 r(\infty)$. From formula (61), the solution of the equation $(65), w(t, x)$, with initial value $w(0, x)=u(0, x)-r(-\infty)-\epsilon_{0}$ satisfies the integral equation

$$
\begin{aligned}
w(t, x)= & \sum_{m=-\infty}^{+\infty} e^{-(\rho+2 D) t} I_{m}(2 D t) w(0, x+m) \\
& +\int_{0}^{t} \sum_{m=-\infty}^{+\infty} e^{-(\rho+2 D) \tau} I_{m}(2 D \tau) \cdot\left[\left(w(t-\tau, x+m)+r(-\infty)+\epsilon_{0}\right) .\right. \\
& \left(\rho+r(x-c t+c \tau-y)-w(t-\tau, x+m)-r(-\infty)-\epsilon_{0}\right) \\
& \left.-\rho\left(r(-\infty)+\epsilon_{0}\right)\right] d \tau .
\end{aligned}
$$

Since $0 \leq u(t, x) \leq r(\infty)$, it is easy to see that the following expression is bounded:

$$
\begin{aligned}
& \mid\left(w(t-\tau, x+m)+r(-\infty)+\epsilon_{0}\right) . \\
& \left(\rho+r(x-c t+c \tau-y)-w(t-\tau, x+m)-r(-\infty)-\epsilon_{0}\right)-\rho\left(r(-\infty)+\epsilon_{0}\right) \mid \\
& \leq r(\infty)(\rho+2 r(\infty))+\rho\left(r(-\infty)+\epsilon_{0}\right) .
\end{aligned}
$$

Therefore,

$$
\int_{0}^{\infty} \sum_{-\infty}^{+\infty} e^{-(\rho+2 D) \tau} I_{m}(2 D \tau)\left[\left(w(t-\tau, x+m)+r(-\infty)+\epsilon_{0}\right)\right.
$$




$$
\begin{aligned}
& \left.\left(\rho+r(x-c t+c \tau-y)-w(t-\tau, x+m)-r(-\infty)-\epsilon_{0}\right)-\rho\left(r(-\infty)+\epsilon_{0}\right)\right] d \tau \\
& \leq \int_{0}^{\infty} \sum_{-\infty}^{+\infty} e^{-(\rho+2 D) \tau} I_{m}(2 D \tau)\left[r(\infty)(\rho+2 r(\infty))+\rho\left(r(-\infty)+\epsilon_{0}\right)\right] d y d \tau \\
& \leq \int_{0}^{\infty} e^{-\rho \tau} d \tau\left[r(\infty)(\rho+2 r(\infty))+\rho\left(r(-\infty)+\epsilon_{0}\right)\right] \\
& \leq \frac{r(\infty)(\rho+2 r(\infty))+\rho\left(r(-\infty)+\epsilon_{0}\right)}{\rho} .
\end{aligned}
$$

It follows that, for the given $\epsilon_{0}>0$, there exists $\eta>0$ and $A>\eta$, such that

$$
\begin{aligned}
& \int_{0}^{\eta} \sum_{-\infty}^{+\infty} e^{-(\rho+2 D) \tau} I_{m}(2 D \tau)\left[\left(w(t-\tau, x+m)+r(-\infty)+\epsilon_{0}\right)\right. \\
& \left.\left(\rho+r(x-c t+c \tau-y)-w(t-\tau, x+m)-r(-\infty)-\epsilon_{0}\right)-\rho\left(r(-\infty)+\epsilon_{0}\right)\right] d \tau<\epsilon_{0}
\end{aligned}
$$

and

$$
\begin{aligned}
& \int_{A}^{\infty} \sum_{-\infty}^{+\infty} e^{-(\rho+2 D) \tau} I_{m}(2 D \tau)\left[\left(w(t-\tau, x+m)+r(-\infty)+\epsilon_{0}\right)\right. \\
& \left.\left(\rho+r(x-c t+c \tau-y)-w(t-\tau, x+m)-r(-\infty)-\epsilon_{0}\right)-\rho\left(r(-\infty)+\epsilon_{0}\right)\right] d \tau<\epsilon_{0} .
\end{aligned}
$$

For $n>0$, let

$$
\begin{aligned}
& \int_{\eta}^{A} \sum_{-\infty}^{+\infty} e^{-(\rho+2 D) \tau} I_{m}(2 D \tau)\left[\left(w(t-\tau, x+m)+r(-\infty)+\epsilon_{0}\right)\right. \\
& \left.\cdot\left(\rho+r(x-c t+c \tau-y)-w(t-\tau, x+m)-r(-\infty)-\epsilon_{0}\right)-\rho\left(r(-\infty)+\epsilon_{0}\right)\right] d \tau \\
& =I(n)+I I(n),
\end{aligned}
$$

where

$$
\begin{aligned}
& I(n)=\int_{\eta}^{A} \sum_{|m| \leq n-c \tau} e^{-(\rho+2 D) \tau} I_{m}(2 D \tau)\left[\left(w(t-\tau, x+m)+r(-\infty)+\epsilon_{0}\right)\right. \\
& \left.\cdot\left(\rho+r(x-c t+c \tau-y)-w(t-\tau, x+m)-r(-\infty)-\epsilon_{0}\right)-\rho\left(r(-\infty)+\epsilon_{0}\right)\right] d \tau,
\end{aligned}
$$

and

$$
\begin{aligned}
& I I(n)=\int_{\eta}^{A} \sum_{|m|>n-c \tau} e^{-(\rho+2 D) \tau} I_{m}(2 D \tau)\left[\left(w(t-\tau, x+m)+r(-\infty)+\epsilon_{0}\right)\right. \\
& \left.\cdot\left(\rho+r(x-c t+c \tau-y)-w(t-\tau, x+m)-r(-\infty)-\epsilon_{0}\right)-\rho\left(r(-\infty)+\epsilon_{0}\right)\right] d \tau .
\end{aligned}
$$

By assuming $n>(c+2 D) A$ and using Lemma IV.1, we derive that

$$
I(n) \leq \int_{\eta}^{A} e^{-\rho \tau} \sum_{|m| \geq n-c \tau} \frac{C}{\sqrt{2 \pi}} \frac{e^{-\alpha_{2} m}}{\left((2 D \tau)^{2}+m^{2}\right)^{1 / 4}}\left[\left(w(t-\tau, x+m)+r(-\infty)+\epsilon_{0}\right)\right.
$$




$$
\begin{aligned}
& \left.\left(\rho+r(x-c t+c \tau-y)-w(t-\tau, x+m)-r(-\infty)-\epsilon_{0}\right)-\rho\left(r(-\infty)+\epsilon_{0}\right)\right] d \tau \\
\leq & \int_{\eta}^{A} e^{-\rho \tau} \sum_{|m| \geq n-c \tau} \frac{C}{\sqrt{2 \pi}} \frac{e^{-\alpha_{2} m}}{\left((2 D \tau)^{2}+m^{2}\right)^{1 / 4}}\left[r(\infty)(\rho+2 r(\infty))+\rho\left(r(-\infty)+\epsilon_{0}\right)\right] d \tau \\
\leq & \int_{\eta}^{A} \frac{C}{\sqrt{4 \pi D A}} e^{-\rho \tau} \sum_{|m| \geq n-c \tau} e^{-\alpha_{2} m}\left[r(\infty)(\rho+2 r(\infty))+\rho\left(r(-\infty)+\epsilon_{0}\right)\right] d \tau \\
\leq & \int_{\eta}^{A} \frac{C}{\sqrt{4 \pi D A}} e^{-\rho \tau}\left[r(\infty)(\rho+2 r(\infty))+\rho\left(r(-\infty)+\epsilon_{0}\right)\right] \int_{n-c \tau}^{\infty} e^{-\alpha_{1} m} d m d \tau .
\end{aligned}
$$

Since $\int_{n-c \tau}^{\infty} e^{-\alpha_{1} m} d m$ uniformly converges to 0 when $n$ is sufficiently large, for the given $\epsilon_{0}$, there exists sufficiently large $n_{0}$ such that

$$
I\left(n_{0}\right)<\epsilon_{0}
$$

Now we are going to prove that $I I\left(n_{0}\right)<\epsilon_{0}(r(-\infty)+\epsilon)$. By the monotonicity of $r(x)$, there is sufficiently large $M_{1}>0$, such that for $x<-M_{1}+c t$,

$$
r\left(x-c t+n_{0}\right)<r(-\infty)+\epsilon_{0} .
$$

From Lemma IV.3, $\bar{u}(t, x)$ is nonincreasing in $t$ and nondecreasing in $x, \bar{u}(t,-\infty)=$ $r(-\infty)$, and $\bar{u}(t, \infty)=r(\infty)$ for $t>0$. It follows that, for the given $\epsilon_{0}$, there are sufficiently large $T_{2}>0$ and $M_{2}>0$, such that for $t>A+T_{2}$, and $x<-M_{2}$,

$$
\bar{u}\left(t-A, x-c A+m_{0}\right)<r(-\infty)+\epsilon_{0} .
$$

By the definition of $I I\left(n_{0}\right)$, its integration variables $\tau$ and $y$ satisfy $c \tau-y \leq n_{0}$. We have that

$$
\begin{aligned}
I I\left(n_{0}\right)= & \int_{\eta}^{A} \sum_{|m| \leq n_{0}-c \tau} e^{-(\rho+2 D) \tau} I_{m}(2 D \tau)\left[\left(w(t-\tau, x+m)+r(-\infty)+\epsilon_{0}\right)\right. \\
& \left.\left(\rho+r(x-c t+c \tau+m)-w(t-\tau, x+m)-r(-\infty)-\epsilon_{0}\right)-\rho\left(r(-\infty)+\epsilon_{0}\right)\right] d \tau \\
= & \int_{\eta}^{A} \sum_{|m| \leq n_{0}-c \tau} e^{-(\rho+2 D) \tau} I_{m}(2 D \tau)[u(t-\tau, x+m) \\
& \left.(\rho+r(x-c t+c \tau+m)-u(t-\tau, x+m))-\rho\left(r(-\infty)+\epsilon_{0}\right)\right] d \tau \\
\leq & \int_{\eta}^{A} \sum_{|m| \leq n_{0}-c \tau} e^{-(\rho+2 D) \tau} I_{m}(2 D \tau)[\bar{u}(t-\tau, x+m) \\
& \left.(\rho+r(x-c t+c \tau+m)-\bar{u}(t-\tau, x+m))-\rho\left(r(-\infty)+\epsilon_{0}\right)\right] d \tau \\
\leq & \int_{\eta}^{A} \sum_{|m| \leq n_{0}-c \tau} e^{-(\rho+2 D) \tau} I_{m}(2 D \tau)\left[\bar{u}\left(t-A, x+n_{0}-c A\right)\right.
\end{aligned}
$$




$$
\begin{aligned}
& \left.\left(\rho+r\left(x-c t+n_{0}\right)-r(-\infty)\right)-\rho\left(r(-\infty)+\epsilon_{0}\right)\right] d \tau \\
\leq & \int_{\eta}^{A} \sum_{|m| \leq n_{0}-c \tau} e^{-(\rho+2 D) \tau} I_{m}(2 D \tau)\left[\left(r(-\infty)+\epsilon_{0}\right)\left(\rho+\epsilon_{0}\right)-\rho\left(r(\infty)+\epsilon_{0}\right)\right] d \tau \\
\leq & \left(r(-\infty)+\epsilon_{0}\right) \epsilon_{0} \int_{\eta}^{A} e^{-\rho \tau} d \tau \\
\leq & \left(r(-\infty)+\epsilon_{0}\right) \epsilon_{0} .
\end{aligned}
$$

Since $|w(0, x)|=\left|u(0, x)-r(-\infty)-\epsilon_{0}\right|<r(\infty)-r(-\infty)-\epsilon_{0}$,

$$
\sum_{m=-\infty}^{+\infty} e^{-(\rho+2 D) t} I_{m}(2 D t) w(0, x+m) \leq\left(r(\infty)-r(-\infty)-\epsilon_{0}\right) e^{-\rho t} .
$$

We have that, for the given $\epsilon_{0}$, there exists $T_{3}>0$, such that for $t>T_{3}$,

$$
\sum_{m=-\infty}^{+\infty} e^{-(\rho+2 D) t} I_{m}(2 D t) w(0, x+m) \leq \epsilon_{0}
$$

Therefore, for $t \geq T:=\max \left\{T_{2}, T_{3}\right\}$ and $x \leq-M+c t$ with $M:=\max \left\{M_{1}, M_{2}\right\}$,

$$
w(t, x) \leq \epsilon_{0}\left(4+r(-\infty)+\epsilon_{0}\right)=\epsilon,
$$

which implies that

$$
u(t, x) \leq r(-\infty)+\epsilon
$$

In the case of $c<0$, let $\bar{u}(t, x)$ be the solution of model $(64)$ with $\bar{u}(0, x)=$ $r(\infty)$. From Lemma IV.3, $\bar{u}(t,-\infty)=r(-\infty)$. Then for any $\epsilon>0$, there exists $T_{1}>0$ and $M>0$ such that for $x<-M, \bar{u}\left(T_{1}, x\right)<r(-\infty)+\epsilon$. Since $\bar{u}(t, x)$ is nonincreasing in $t$, we have that

$$
\bar{u}(t, x)<r(-\infty)+\epsilon \text { for } t \geq T_{1} \text { and } x \leq-M .
$$

The function $\check{u}=\bar{u}(t, x-c t)$ satisfies

$$
\frac{\partial \check{u}}{\partial t}=D[\check{u}(t, x+1)-2 \check{u}(t, x)+\check{u}(t, x-1)]-c[\check{u}(t, x+1)-\check{u}(t, x)]+\check{u}(r(x-c t)-\check{u}) .
$$

Since $c<0$, and $\bar{u}(t, x)$ is nondecreasing in $x$, we have that

$$
\frac{\partial \check{u}}{\partial t} \geq D[\check{u}(t, x+1)-2 \check{u}(t, x)+\check{u}(t, x-1)]+\check{u}(r(x-c t)-\check{u}) .
$$

So $\breve{u}(t, x)$ is an upper solution of model (58). Let $u(0, x) \leq r(\infty)$, we have that $u(t, x) \leq \check{u}(t, x)$. It yields that

$$
u(t, x)<r(-\infty)+\epsilon \text { for } t \geq T_{1} \text { and } x \leq-M+c t
$$


The proof is complete.

We introduce the following lemma regarding two upper solutions for model (58).

Lemma IV.5 Let $u(t, x)$ is a solution of model (58). Then

(a) If $u(0, x)$ is zero for all sufficiently large $x$, and $0 \leq u(0, x) \leq r(\infty)$, then for any small positive $\epsilon$ there exist positive numbers $A$ and $\mu_{\epsilon}$ such that

$$
u(t, x) \leq A e^{-\mu_{\epsilon}\left(x-\left(c^{*}(\infty)+\epsilon\right) t\right)} .
$$

(b) Assume $c>-c^{*}(-\infty)$. If $u(0, x)$ is zero for all sufficiently negative $x$, and $0 \leq u(0, x) \leq r(\infty)$, then for any positive $\epsilon_{1}$, there exist positive numbers $B$ and $\mu_{1}$ such that for all $t>0$ and $-\infty<x<\infty$,

$$
u(t, x) \leq w(t, x)=B e^{\mu_{1}\left(x+\left(c^{*}(-\infty)+\epsilon_{1}\right) t\right)} .
$$

Proof. We first prove (a). For any $\epsilon>0$, since $\phi(x ; \mu)$ decreases in $0<\mu<$ $\mu^{*}(x)$, there exists $\mu_{\epsilon}$ such that $\phi\left(\infty ; \mu_{\epsilon}\right)=c^{*}(\infty)+\epsilon$. We assume that $A$ is a positive number and

$$
\hat{u}(t, x)=A e^{-\mu_{\epsilon}\left(x-\left(c^{*}(\infty)+\epsilon\right) t\right)} .
$$

It is easy to show that $\hat{u}(t, x)$ is a solution of

$$
\frac{\partial u}{\partial t}=D[u(t, x+1)-2 u(t, x)+u(t, x-1)]+r(\infty) u \text {. }
$$

Since $r(\infty) u \geq u(r(x-c t)-u), \hat{u}(t, x)$ is an upper solution of model (58). Choose $A$ sufficiently large such that $u(0, x) \leq \hat{u}(0, x)=A e^{-\mu_{\epsilon} x}$. Then

$$
u(t, x) \leq \hat{u}(t, x)=A e^{-\mu_{\epsilon}\left(x-\left(c^{*}(\infty)+\epsilon\right) t\right)} .
$$

We now prove statement (b). By the definition of $c^{*}(x)$, we have that

$$
\begin{aligned}
c^{*}(-\infty) & =\inf _{\mu>0} \frac{4 D \sinh ^{2}(\mu / 2)+r(-\infty)}{\mu} \\
& =\inf _{\mu>0} \frac{4 D\left(\frac{e^{\mu / 2}-e^{-\mu / 2}}{2}\right)^{2}+r(-\infty)}{\mu} \\
& =\inf _{\mu>0} \frac{D\left(e^{\mu}-2+e^{-\mu}\right)+r(-\infty)}{\mu} \\
& =\liminf _{\delta \rightarrow 0} \frac{D\left(e^{\mu}-2+e^{-\mu}\right)+r(-\infty)+\delta}{\mu} .
\end{aligned}
$$


Therefore, for any $\epsilon_{1}>0$, there exists $\delta>0$ and $0<\mu_{1}<\mu^{*}(-\infty)$, such that

$$
c^{*}(-\infty)+\frac{\epsilon_{1}}{2}=\frac{2 D\left(e^{\mu_{1}}-2+e^{-\mu_{1}}\right)+r(-\infty)+\delta}{\mu_{1}} .
$$

It follows that

$$
\mu_{1}\left(c^{*}(-\infty)+\frac{\epsilon_{1}}{2}\right)=2 D\left(e^{\mu_{1}}-2+e^{-\mu_{1}}\right)+r(-\infty)+\delta
$$

Since $r(x)$ is continuous and nondecreasing in $-\infty<x<\infty$, there exists $x_{1}$ such that if $x<x_{1}$, then

$$
r(x) \leq r(-\infty)+\delta
$$

It follows that for any $t>0$, if $x<x_{1}+c t$, then

$$
r(x-c t) \leq r(-\infty)+\delta
$$

Additionally, there exists $B$ sufficiently large such that $B e^{\mu_{1} x_{1}}=r(\infty)$.

Let

$$
S[u](t, x)=\frac{\partial u}{\partial t}-D[u(t, x+1)-2 u(t, x)+u(t, x-1)]-r(x-c t) u+u^{2} .
$$

Then

$$
\begin{aligned}
S[w](t, x) & =\frac{\partial w}{\partial t}-D[w(t, x+1)-2 w(t, x)+w(t, x-1)]-r(x-c t) w+w^{2} \\
& =w(t, x)\left[\mu_{1}\left(c^{*}(-\infty)+\epsilon_{1}\right)-D\left(e^{\mu}-2-e^{-\mu}\right)-r(x-c t)+w(t, x)\right] \\
& =w(t, x)\left[r(-\infty)+\epsilon-r(x-c t)+B e^{\mu_{1}\left(x+\left(c^{*}(-\infty)+\epsilon_{1}\right) t\right)}\right] .
\end{aligned}
$$

It follows that for any $t>0$, when $x<x_{1}+c t$, we have that,

$$
S[w](t, x) \geq w(t, x) B e^{\mu_{1}\left(x+\left(c^{*}(-\infty)+\epsilon_{1}\right) t\right)}>0,
$$

and when $x>x_{1}+c t$, we have that,

$$
B e^{\mu_{1}\left(x+\left(c^{*}(-\infty)+\epsilon_{1}\right) t\right)} \geq B e^{\mu_{1}\left(x_{1}+c t+\left(c^{*}(-\infty)+\epsilon_{1}\right) t\right)} \geq B e^{\mu_{1} x_{1}}=r(\infty) .
$$

So $S[w](t, x)>0$ for any $t>0$ and $-\infty<x<\infty$.

Therefore, $w(t, x)$ is an upper solution of $u(t, x)$, i.e.

$$
u(t, x) \leq B e^{\mu_{1}\left(x+\left(c^{*}(-\infty)+\epsilon_{1}\right) t\right)} .
$$

The proof is complete.

The next lemma is necessary in proving theorems in section 5 . 
Lemma IV.6 Assume $c$ and $\mu$ are constants. For any $\epsilon>0$ sufficiently small, there exist $b>0$ sufficiently large and $S_{0}$ a positive number such that for any real number $x$,

$$
\begin{aligned}
& \int_{0}^{t} \sum_{m=-\infty}^{+\infty} e^{-(\rho+2 D) \tau} I_{m}(2 D \tau) e^{\mu m} r(x-m-c(t-\tau)) d \tau \\
& \leq\left(1+S_{0} \epsilon\right) \int_{0}^{t} \sum_{c \tau-b}^{\infty} e^{-(\rho+2 D) \tau} I_{m}(2 D \tau) e^{\mu m} r(x-m-c(t-\tau)) d \tau
\end{aligned}
$$

where $I_{m}(2 D \tau)$ is defined by the formula (52).

Proof. Choose $\eta, A$ and $h$ to be any positive numbers. When $\mu>0$, let

$$
\int_{0}^{t} \sum_{m=-\infty}^{+\infty} e^{-(\rho+2 D) \tau} I_{m}(2 D \tau) e^{\mu m} r(x-m-c(t-\tau)) d \tau=\mathrm{I}(\eta)+\mathrm{I}(A)+\mathrm{I}(h)+\mathrm{II}(h),
$$

where

$$
\begin{aligned}
\mathrm{I}(A) & =\int_{A}^{\infty} \sum_{m=-\infty}^{+\infty} e^{-(\rho+2 D) \tau} I_{m}(2 D \tau) e^{\mu m} r(x-m-c(t-\tau)) d \tau, \\
\mathrm{I}(\eta) & =\int_{0}^{\eta} \sum_{m=-\infty}^{+\infty} e^{-(\rho+2 D) \tau} I_{m}(2 D \tau) e^{\mu m} r(x-m-c(t-\tau)) d \tau, \\
\mathrm{I}(h) & =\int_{\eta}^{A} \sum_{-\infty}^{c \tau-h} e^{-(\rho+2 D) \tau} I_{m}(2 D \tau) e^{\mu m} r(x-m-c(t-\tau)) d \tau, \\
\mathrm{II}(h) & =\int_{\eta}^{A} \sum_{c \tau-h}^{\infty} e^{-(\rho+2 D) \tau} I_{m}(2 D \tau) e^{\mu m} r(x-m-c(t-\tau)) d \tau .
\end{aligned}
$$

Here we have omitted explicitly listing the $x$ dependency of I and II. We have that

$$
\begin{aligned}
\mathrm{I}(A) & \leq \int_{A}^{\infty} \sum_{m=-\infty}^{+\infty} e^{-(\rho+2 D) \tau} I_{m}(2 D \tau) e^{\mu m} r(\infty) d \tau \\
& \leq r(\infty) \int_{A}^{\infty} e^{-(\rho+2 D) \tau}\left[\sum_{m=0}^{+\infty} I_{m}(2 D \tau) e^{\mu m}+\sum_{m=-\infty}^{-1} I_{m}(2 D \tau) e^{\mu m}\right] d \tau \\
& \leq r(\infty) \int_{A}^{\infty} e^{-(\rho+2 D) \tau}\left[\sum_{m=0}^{+\infty} I_{m}(2 D \tau) e^{\mu m}+\sum_{m=1}^{\infty} I_{m}(2 D \tau) e^{-\mu m}\right] d \tau \\
& \leq r(\infty) \int_{A}^{\infty} e^{-(\rho+2 D) \tau}\left[\sum_{m=0}^{+\infty} I_{m}\left(2 D e^{\mu} \tau\right)+\sum_{m=1}^{\infty} I_{m}(2 D \tau)\right] d \tau \\
& \leq r(\infty) \int_{A}^{\infty} e^{-(\rho+2 D) \tau}\left[e^{2 D e^{\mu} \tau}+e^{2 D \tau}\right] d \tau \\
& =r(\infty)\left[\frac{e^{-\left(\rho+2 D-2 D e^{\mu}\right) A}}{\rho+2 D-2 D e^{\mu}}+\frac{e^{-\rho A}}{\rho}\right]
\end{aligned}
$$


and

$$
\begin{aligned}
\mathrm{I}(\eta) & \leq \int_{0}^{\eta} \sum_{m=-\infty}^{+\infty} e^{-(\rho+2 D) \tau} I_{m}(2 D \tau) e^{\mu m} r(\infty) d \tau \\
& \leq r(\infty) \int_{0}^{\eta} e^{-(\rho+2 D) \tau}\left[\sum_{m=0}^{+\infty} I_{m}\left(2 D e^{\mu} \tau\right)+\sum_{m=1}^{\infty} I_{m}(2 D \tau)\right] d \tau \\
& \leq r(\infty) \int_{0}^{\eta} e^{-(\rho+2 D) \tau}\left[e^{2 D e^{\mu} \tau}+e^{2 D \tau}\right] d \tau \\
& =r(\infty)\left[\frac{1-e^{-\left(\rho+2 D-2 D e^{\mu}\right) \eta}}{\rho+2 D-2 D e^{\mu}}+\frac{1-e^{-\rho \eta}}{\rho}\right]
\end{aligned}
$$

We assume $\rho$ is sufficiently large such that $\rho+2 D-2 D e^{\mu}>0$, then for any positive $\epsilon$, there exist $\eta$ sufficiently small and $A$ sufficiently large, such that $\mathrm{A}(\eta)<\frac{\epsilon}{4}$ and $\mathrm{I}(\eta)<\frac{\epsilon}{4}$

Assuming $h>(c+2 D) A$ in $\mathrm{I}(h)$, we have that

$$
\begin{aligned}
\mathrm{I}(h) & \leq \int_{\eta}^{A} \sum_{m=-\infty}^{c \tau-h} e^{-(\rho+2 D) \tau} I_{m}(2 D \tau) e^{\mu m} r(\infty) d \tau \\
& \leq \int_{\eta}^{A} r(\infty) \sum_{m=h-c \tau}^{\infty} e^{-(\rho+2 D) \tau} I_{m}(2 D \tau) e^{-\mu m} d \tau \\
& \leq \int_{\eta}^{A} r(\infty) \sum_{m=h-c \tau}^{\infty} e^{-(\rho+2 D) \tau} I_{m}(2 D \tau) d \tau \\
& \leq \int_{\eta}^{A} r(\infty) \sum_{m=h-c \tau}^{\infty} e^{-\rho \tau} \frac{C}{\sqrt{2 \pi}} \frac{e^{-\alpha_{2} m}}{\left((2 D \tau)^{2}+m^{2}\right)^{1 / 4}} d \tau \\
& \leq \int_{\eta}^{A} r(\infty) \sum_{m=h-c \tau}^{\infty} e^{-\rho \tau} \frac{C}{\sqrt{2 \pi}} \frac{e^{-\alpha_{2} m}}{\sqrt{2 D \tau}} d \tau
\end{aligned}
$$

Since $m=h-c \tau \rightarrow \infty$ uniformly for $\tau \in[\eta, A]$ as $h \rightarrow \infty$, for the given $\epsilon>0$, there exists sufficiently large $h=b$ such that

$$
\mathrm{I}(b)<\frac{\epsilon}{4}
$$

Additionally,

$$
\begin{aligned}
\mathrm{II}(b) & \leq \int_{\eta}^{A} \sum_{m=c \tau-h}^{\infty} e^{-(\rho+2 D) \tau} I_{m}(2 D \tau) e^{\mu m} r(\infty) d \tau \\
& \leq \int_{\eta}^{A} \sum_{m=-\infty}^{\infty} e^{-(\rho+2 D) \tau} I_{m}(2 D \tau) e^{\mu m} r(\infty) d \tau \\
& \leq \int_{\eta}^{A} r(\infty) e^{-(\rho+2 D) \tau}\left[\sum_{m=-\infty}^{-1} I_{m}(2 D \tau) e^{\mu m}+\sum_{m=0}^{\infty} I_{m}(2 D \tau) e^{\mu m}\right] d \tau
\end{aligned}
$$




$$
\begin{aligned}
& \leq \int_{\eta}^{A} r(\infty) e^{-(\rho+2 D) \tau}\left[e^{2 D e^{\mu} \tau}+e^{2 D \tau}\right] d \tau \\
& \leq r(\infty) \int_{0}^{\eta} e^{-(\rho+2 D) \tau}\left[e^{2 D e^{\mu} \tau}+e^{2 D \tau}\right] d \tau \\
& =r(\infty)\left[\frac{e^{-\left(\rho+2 D-2 D e^{\mu}\right) \eta-e^{-\left(\rho+2 D-2 D e^{\mu}\right) A}}}{\rho+2 D-2 D e^{\mu}}+\frac{e^{-\rho \eta}-e^{-\rho A}}{\rho}\right] .
\end{aligned}
$$

Similarly, we can prove that

$$
\begin{aligned}
\mathrm{II}(b) & \geq \int_{\eta}^{A} \sum_{m=c \tau-h}^{\infty} e^{-(\rho+2 D) \tau} I_{m}(2 D \tau) e^{\mu m} r(-\infty) d \tau \\
& \geq \int_{\eta}^{A} \sum_{m=0}^{\infty} e^{-(\rho+2 D) \tau} I_{m}(2 D \tau) e^{\mu m} r(-\infty) d \tau \\
& \geq \int_{\eta}^{A} r(-\infty) e^{-(\rho+2 D) \tau}\left[\sum_{m=0}^{\infty} I_{m}(2 D \tau)\right] d \tau \\
& \geq \int_{\eta}^{A} \frac{r(-\infty)}{2} e^{-(\rho+2 D) \tau} e^{2 D \tau} d \tau \\
& =\frac{r(-\infty)}{2 \rho}\left(e^{-\rho \eta}-e^{-\rho A}\right) .
\end{aligned}
$$

It follows that $\operatorname{II}(b)$ is positive and bounded. For the given $\epsilon$, there exists a positive number $S_{0}$ such that

$$
\mathrm{I}(A)+\mathrm{I}(\eta)+\mathrm{I}(b)<S_{0} \epsilon \mathrm{II}(b) .
$$

Therefore,

$$
\begin{aligned}
& \int_{0}^{t} \sum_{m=-\infty}^{+\infty} e^{-(\rho+2 D) \tau} I_{m}(2 D \tau) e^{\mu m} r(x-m-c(t-\tau)) d \tau \\
& \leq\left(1+S_{0} \epsilon\right) \int_{0}^{t} \sum_{c \tau-b}^{\infty} e^{-(\rho+2 D) \tau} I_{m}(2 D \tau) e^{\mu m} r(x-m-c(t-\tau)) d \tau .
\end{aligned}
$$

Similarly, we can prove that when $\mu<0$, equation (67) is also valid. The proof is complete.

\section{Lower Solutions}

Now we construct lower solutions for model (58) by extending the work in $\mathrm{Hu}$ and $\mathrm{Li}(2015)$. We recall the functions $v(\mu ; x)$ to build the lower solution of system (58), which was used in $\mathrm{Hu}$ and $\mathrm{Li}$ (2015). The function $v(\mu ; x)$ is given by

$$
v(\mu ; x)= \begin{cases}e^{-\mu x} \sin \gamma x, & \text { if } 0 \leq x \leq \frac{\pi}{\gamma} \\ 0, & \text { elsewhere }\end{cases}
$$


with $\gamma>0$ and $\eta>0$. This function is continuous and second order derivative in $x$ except at the point $x=0$ and $x=\frac{\pi}{\gamma}$, which was initially introduced in Weinberger (1982). The maximum of $v(\mu ; x)$ occurs at $\sigma(\mu)=\frac{1}{\gamma} \tan ^{-1}\left(\frac{\gamma}{\mu}\right)$. The function $\sigma(\mu)$ is strictly decreasing in $\mu$. We also need the function

$$
v_{-}(\mu ; x)=v(\mu ;-x)
$$

which was used in Weinberger (1982) as well.

We extend the definition of spreading speed by assuming

$$
c_{\gamma}^{*}(\ell)=\inf _{\mu>0} \phi_{\gamma}(\mu, \ell)
$$

where

$$
\phi_{\gamma}(\mu, \ell)=\frac{D\left[\left(e^{\mu}+e^{-\mu}\right) \cos \gamma-2\right]+r(\ell)}{\mu} .
$$

It is easy to prove that $\phi_{\gamma}(\mu, \ell)<\phi(\mu, \ell)$ and $\phi_{\gamma}(\mu, \ell)$ converge to $\phi(\mu, \ell)$ uniformly for $\mu$ in any bounded interval as $\gamma>0$. It follows that $c_{\gamma}^{*}(\ell)<c^{*}(\ell)$ and $c_{\gamma}^{*}(\ell) \rightarrow c^{*}(\ell)$ as $\gamma \rightarrow 0$. Define

$$
\psi(\mu, \gamma)=\frac{D \sin \gamma}{\gamma}\left(e^{\mu}-e^{-\mu}\right)
$$

For the positive number $\alpha$ and $\ell$ satisfying $c^{*}(\ell)=c^{*}(\infty)-\epsilon$, we recall the function defined in $\mathrm{Hu}$ and $\mathrm{Li}$ (2015) as follows:

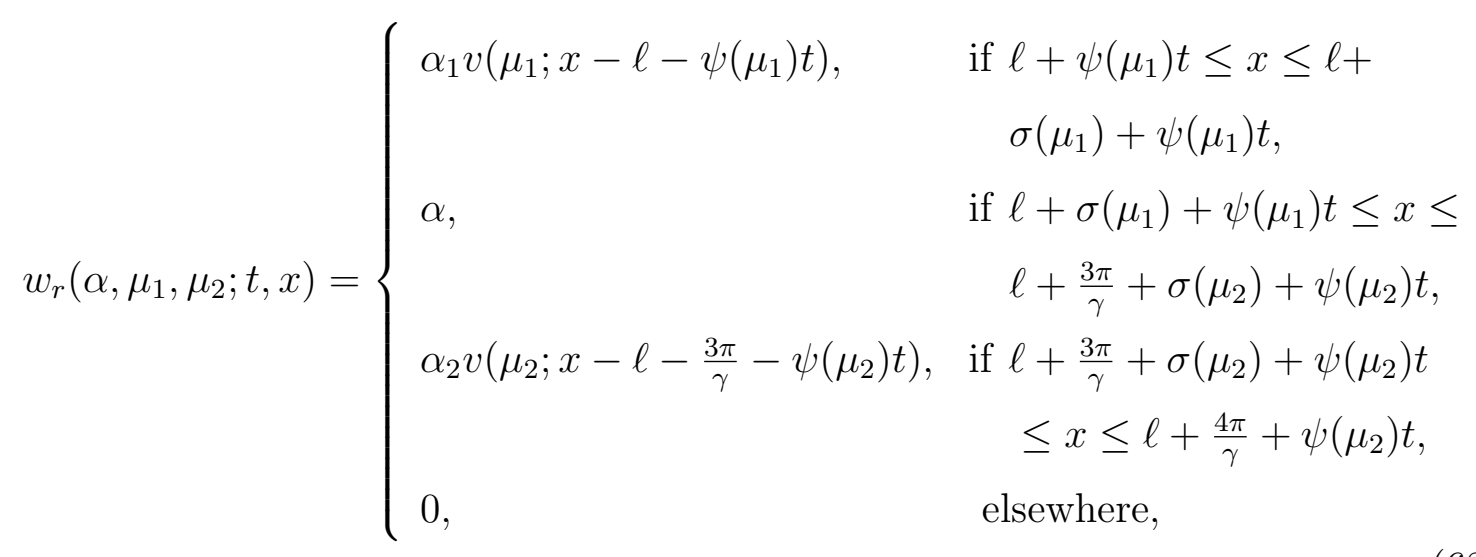

where $\alpha_{1}=\frac{\alpha}{v\left(\mu_{1} ; \sigma\left(\mu_{1}\right)\right)}, \alpha_{2}=\frac{\alpha}{v\left(\mu_{2} ; \sigma\left(\mu_{2}\right)\right)}$ and $\psi(\mu)$ is defined by formula (63). For $x$ in the interval

$$
\left[\ell+\sigma\left(\mu_{1}\right)+\psi\left(\mu_{1}\right) t, \ell+\frac{3 \pi}{\gamma}+\sigma\left(\mu_{2}\right)+\psi\left(\mu_{2}\right) t\right],
$$


we have $w_{r}\left(\alpha, \mu_{1}, \mu_{2} ; t, x\right)=\alpha$, with the end points shifting rightward at speeds $\psi\left(\mu_{1}\right)$ and $\psi\left(\mu_{2}\right)$ as $t \rightarrow \infty$.

Similar to the reaction-diffusion system, we define

$$
w\left(\alpha, \mu_{1}, \mu_{2} ; t, x\right)=\left\{\begin{array}{lc}
\alpha_{1} v_{-}\left(\mu_{1} ; x-\ell+\psi\left(\mu_{1}\right) t\right), & \text { if } \ell-\psi\left(\mu_{1}\right) t-\frac{\pi}{\gamma} \leq x \leq \ell- \\
\alpha, & \sigma\left(\mu_{1}\right)-\psi\left(\mu_{1}\right) t, \\
& \text { if } \ell-\sigma\left(\mu_{1}\right)-\psi\left(\mu_{1}\right) t \leq x \leq \\
& \ell+\frac{3 \pi}{\gamma}+\sigma\left(\mu_{2}\right)+\psi\left(\mu_{2}\right) t, \\
\alpha_{2} v\left(\mu_{2} ; x-\ell-\frac{3 \pi}{\gamma}-\psi\left(\mu_{2}\right) t\right), & \text { if } \ell+\frac{3 \pi}{\gamma}+\sigma\left(\mu_{2}\right)+\psi\left(\mu_{2}\right) t \\
& \leq x \leq \ell+\frac{4 \pi}{\gamma}+\psi\left(\mu_{2}\right) t, \\
0, & \text { elsewhere, }
\end{array}\right.
$$

and

$$
w_{l}\left(\alpha, \mu_{1}, \mu_{2} ; t, x\right)=\left\{\begin{array}{lr}
\alpha_{1} v_{-}\left(\mu_{1} ; x-\ell+\psi\left(\mu_{1}\right) t\right), & \text { if } \ell-\psi\left(\mu_{1}\right) t-\frac{\pi}{\gamma} \leq x \leq \ell- \\
\alpha, & \sigma\left(\mu_{1}\right)-\psi\left(\mu_{1}\right) t, \\
& \text { if } \ell-\sigma\left(\mu_{1}\right)-\psi\left(\mu_{1}\right) t \leq x \leq \\
& \ell+\frac{3 \pi}{\gamma}-\sigma\left(\mu_{2}\right)-\psi\left(\mu_{2}\right) t \\
\alpha_{2} v_{-}\left(\mu_{2} ; x-\ell-\frac{3 \pi}{\gamma}+\psi\left(\mu_{2}\right) t\right), & \text { if } \ell+\frac{3 \pi}{\gamma}-\sigma\left(\mu_{2}\right)-\psi\left(\mu_{2}\right) t \\
& \leq x \leq \ell+\frac{3 \pi}{\gamma}-\psi\left(\mu_{2}\right) t \\
0, & \text { elsewhere. }
\end{array}\right.
$$

We have that $w\left(\alpha, \mu_{1}, \mu_{2} ; t, x\right)=\alpha$ when $x$ in the interval

$$
\left[\ell-\sigma\left(\mu_{1}\right)-\psi\left(\mu_{1}\right) t, \ell+\frac{3 \pi}{\gamma}+\sigma\left(\mu_{2}\right)+\psi\left(\mu_{2}\right) t\right]
$$

with left end points shifting leftward at speeds $\psi\left(\mu_{1}\right)$ and right end point shifting rightward at speeds $\psi\left(\mu_{2}\right)$ as $t \rightarrow \infty$. When $x$ in the interval

$$
\left[\ell-\sigma\left(\mu_{1}\right)-\psi\left(\mu_{1}\right) t, \ell+\frac{3 \pi}{\gamma}-\sigma\left(\mu_{2}\right)-\psi\left(\mu_{2}\right) t\right],
$$

we have $w_{l}\left(\alpha, \mu_{1}, \mu_{2} ; t, x\right)=\alpha$, with both end points shifting leftward at speeds $\psi\left(\mu_{1}\right)$ and $\psi\left(\mu_{2}\right)$ as $t \rightarrow \infty$.

The following lemma was given in $\mathrm{Hu}$ and $\mathrm{Li}$ (2015) (Lemma 5.1) for $-\infty<$ $r(-\infty)<0$, but is valid for the case of $0<r(-\infty)<r(\infty)$ as well. 
Lemma IV.7 Assume that Hypothesis IV.1 is satisfied and $0 \leq c<c^{*}(\infty)$. Assume also $u(t, x)$ is a solution of model (58) with $0<u(0, x) \leq r(\infty)$ for all $x$. For any $\epsilon$ satisfying $0<\epsilon<\frac{c^{*}(\infty)-c}{5}$, there exist $\ell$ such that $c^{*}(\ell)=c^{*}(\infty)-\epsilon$, and $\gamma>0$ such that $c^{*}(\ell)-c_{\gamma}^{*}(\ell) \leq \epsilon$. Let $0<\mu_{1}<\mu_{2}<\mu^{*}(\ell)$ with $\psi\left(\mu_{1}, \gamma\right)=c+\epsilon$ and $\psi\left(\mu_{2}, \gamma\right)=c_{\gamma}^{*}(\ell)-\epsilon$. Then for $\mu \in\left[\mu_{1}, \mu_{2}\right]$, and $0<a \leq \epsilon / \mu_{2}$, the function $\omega(t, x)=$ av $(\mu ; x-\ell-\psi(\mu, \gamma) t)$ is a continuous lower solution of model (58). Furthermore if $u(0, x) \geq a v(\mu, x-\ell)$, then $u(t, x) \geq a v(\mu, x-\ell-\psi(\mu) t)$ for all $t>0$.

The proof of this lemma is similar to the proof for Lemma 5.1 in $\mathrm{Hu}$ and $\mathrm{Li}$ (2015), so we omit the details.

Similar to Lemma IV.7, the following lemma is about lower solutions of model (58) when $c \geq c^{*}(\infty)$.

Lemma IV.8 Assume that Hypothesis IV.1 is satisfied and $c \geq c^{*}(\infty)$. Assume also $u(t, x)$ is a solution of model (58) with $0<u(0, x) \leq r(\infty)$ for all $x$. For any positive $\epsilon$, there exists $\ell$ such that $c^{*}(\ell)=c^{*}(\infty)-\epsilon$, and $\gamma>0$ such that $c^{*}(\ell)-c_{\gamma}^{*}(\ell) \leq \epsilon$. Let $0<\mu_{1}=\mu_{2}<\mu^{*}(\ell)$ with $\psi\left(\mu_{1}, \gamma\right)=\psi\left(\mu_{2}, \gamma\right)=c_{\gamma}^{*}(-\infty)-\epsilon$. Then for $\mu \in\left[0, \mu_{2}\right]$ and $0<a \leq \epsilon / \mu_{2}$, the function $\tilde{u}_{2}(t, x)=a v(\mu ; x-\ell-\psi(\mu, \gamma) t)$ is a continuous lower solution of model (58), in the sense that if $u(0, x) \geq a v(\mu, x-\ell)$, then $u(t, x) \geq a v(\mu, x-\ell-\psi(\mu) t)$ for all $t>0 ;$ for $\mu \in\left[0, \mu_{1}\right]$, and $0<a \leq \epsilon / \mu_{1}$, the function $\tilde{u}_{1}(t, x)=a v_{-}(\mu ; x-\ell+\psi(\mu, \gamma) t)$ is a continuous lower solution of model (58), in the sense that if $u(0, x) \geq a v_{-}(\mu, x-\ell)$, then $u(t, x) \geq a v_{-}(\mu, x-\ell+\psi(\mu) t)$ for all $t>0$.

Proof. From the proof of Lemma 5.1 in $\mathrm{Hu}$ and Li (2015), in order to prove that $\omega(t, x)=\tilde{u}_{2}(t, x)$ is a lower solution of model (58), it will suffice to prove that for $t>0, \ell+\psi(\mu, \gamma) \leq x \leq \ell+\pi / \gamma+\psi(\mu, \gamma) t$

$$
\frac{\partial \omega}{\partial t}(t, x) \leq D[\omega(t, x+1)-2 \omega(t, x)+\omega(t, x-1)]+\omega(t, x)(r(x-c t)-\omega(t, x)) .
$$

When $\omega(t, x)=\tilde{u}_{2}(t, x)$, we have that

$$
\begin{aligned}
\frac{\partial \omega}{\partial t}(t, x)= & a e^{-\mu(x-\ell-\psi(\mu, \gamma) t)} \psi(\mu, \gamma) \\
& (\mu \sin \gamma(x-\ell-\psi(\mu, \gamma) t)-\gamma \cos \gamma(x-\ell-\psi(\mu, \gamma) t))
\end{aligned}
$$


Also,

$$
\begin{aligned}
& D[\omega(t, x+1)-2 \omega(t, x)+\omega(t, x-1)] \\
\geq & D a e^{-\mu_{2}(x-\ell-\psi(\mu, \gamma) t)}\left[\left(e^{-\mu}+e^{\mu}\right) \cos \gamma-2\right] \sin \gamma(x-\ell-\psi(\mu, \gamma) t) \\
& -D a e^{-\mu(x-\ell-\psi(\mu, \gamma) t)} \gamma \psi(\mu, \gamma) \cos \gamma(x-\ell-\psi(\mu, \gamma) t)
\end{aligned}
$$

Equations (71), (72) and (73) show that in order to prove $\omega$ is a lower solution of model (58), we need to verify that

$$
\mu \psi(\mu, \gamma) \leq D\left[\left(e^{-\mu}+e^{\mu}\right) \cos \gamma-2\right]+r(x-c t)-a v(\mu ; x-\ell-\psi(\mu, \gamma) t)
$$

Since $r(x-c t) \geq r(-\infty)$, it suffices to prove that

$$
\mu \psi(\mu, \gamma) \leq D\left[\left(e^{-\mu}+e^{\mu}\right) \cos \gamma-2\right]+r(-\infty)-a
$$

It follows that

$$
\psi(\mu, \gamma) \leq \phi_{\gamma}(\mu,-\infty)-a / \mu_{2}
$$

Therefore, we choose $\mu_{2}$ to satisfy that $\psi\left(\mu_{2}, \gamma\right)=c_{\gamma}^{*}(-\infty)-\epsilon$ with $0<a<\epsilon / \mu_{2}$, so that when $\mu \in\left[0, \mu_{2}\right]$, the function $\tilde{u}_{2}(t, x)=a v(\mu ; x-\ell-\psi(\mu, \gamma) t)$ is a continuous lower solution of model (58), which means that if $u(0, x) \geq a v(\mu, x-\ell)$, then $u(t, x) \geq$ $a v(\mu, x-\ell-\psi(\mu) t)$ for all $t>0$.

Similarly, to prove that $\omega(t, x)=\tilde{u}_{1}(t, x)$ is a lower solution of model (58), it will suffice to prove that for $t>0, \ell-\pi / \gamma-\psi(\mu, \gamma) \leq x \leq \ell-\psi(\mu, \gamma) t$,

$$
\frac{\partial \omega}{\partial t}(t, x) \leq D[\omega(t, x+1)-2 \omega(t, x)+\omega(t, x-1)]+\omega(t, x)(r(x-c t)-\omega(t, x)) .
$$

When $\omega(t, x)=\tilde{u}_{1}(t, x)$, we have that

$$
\begin{aligned}
\frac{\partial \omega}{\partial t}(t, x)= & a e^{\mu(x-\ell+\psi(\mu, \gamma) t)} \psi(\mu, \gamma) . \\
& (-\mu \sin \gamma(x-\ell+\psi(\mu, \gamma) t)-\gamma \cos \gamma(x-\ell+\psi(\mu, \gamma) t)) .
\end{aligned}
$$

Also,

$$
\begin{aligned}
& D[\omega(t, x+1)-2 \omega(t, x)+\omega(t, x-1)] \\
\geq \quad & -D a e^{\mu(x-\ell+\psi(\mu, \gamma) t)}\left[\left(e^{-\mu}+e^{\mu}\right) \cos \gamma-2\right] \sin \gamma(x-\ell+\psi(\mu, \gamma) t) \\
& -D a e^{\mu(x-\ell+\psi(\mu, \gamma) t)} \gamma \psi(\mu, \gamma) \cos \gamma(x-\ell+\psi(\mu, \gamma) t) .
\end{aligned}
$$


Equations (74), (75) and (76) show that, in order to prove $\omega$ is a lower solution of model (58), we need to verify that

$$
\mu \psi(\mu, \gamma) \leq D\left[\left(e^{-\mu}+e^{\mu}\right) \cos \gamma-2\right]+r(x-c t)-a v(\mu ; x-\ell-\psi(\mu, \gamma) t)
$$

Since $r(x-c t) \geq r(-\infty)$, it suffices to prove that

$$
\mu \psi(\mu, \gamma) \leq D\left[\left(e^{-\mu}+e^{\mu}\right) \cos \gamma-2\right]+r(-\infty)-a
$$

It follows that

$$
\psi(\mu, \gamma) \leq \phi_{\gamma}(\mu,-\infty)-a / \mu_{1}
$$

Therefore, we choose $\mu_{1}$ satisfies that $\psi\left(\mu_{1}, \gamma\right)=c_{\gamma}^{*}(-\infty)-\epsilon$ with $0<a<\epsilon / \mu_{1}$, so that when $\mu \in\left[0, \mu_{1}\right]$, the function $\tilde{u}_{1}(t, x)=a v_{-}(\mu ; x-\ell+\psi(\mu, \gamma) t)$ is a continuous lower solution of model (58), which means that if $u(0, x) \geq a v_{-}(\mu, x-\ell)$, then $u(t, x) \geq a v_{-}(\mu, x-\ell+\psi(\mu) t)$ for all $t>0$. The proof is complete.

Similarly, when $c<0$, we have the following lemmas for the lower solution of model (58).

Lemma IV.9 Assume that Hypothesis IV.1 is satisfied and $c^{*}(-\infty)>-c \geq 0$. Assume also $u(t, x)$ is a solution of model (58) with $0<u(0, x) \leq r(\infty)$ for all $x$. For any positive $\epsilon$, there exists $\ell$ such that $c^{*}(\ell)=c^{*}(\infty)-\epsilon$, and $\gamma>0$ such that $c^{*}(\ell)-c_{\gamma}^{*}(\ell) \leq \epsilon$. Let $\psi\left(\mu_{1}\right)=-c-\epsilon, \psi\left(\mu_{2}\right)=c_{\gamma}^{*}(l)-2 \epsilon, \psi\left(\mu_{3}\right)=c^{*}(-\infty)-\epsilon$, and $\psi\left(\mu_{4}\right)=-c+\epsilon$. Then for $\mu \in\left[0, \mu_{2}\right]$, and $0<a \leq \epsilon / \mu_{2}$, the function $\tilde{u}_{2}(t, x)=$ $a v(\mu ; x-\ell-\psi(\mu, \gamma) t)$ is a continuous lower solution of model (58), in the sense that if $u(0, x) \geq a v(\mu, x-\ell)$, then $u(t, x) \geq a v(\mu, x-\ell-\psi(\mu) t)$ for all $t>0$; for $\mu \in\left[0, \mu_{1}\right]$ or $\mu \in\left[\mu_{4}, \mu_{3}\right]$, and $0<a \leq \epsilon / \mu_{3}$, if $u(0, x) \geq a v_{-}(\mu, x-\ell)$, the function $\tilde{u}_{1}(t, x)=a v_{-}(\mu ; x-\ell+\psi(\mu, \gamma) t)$ is a continuous lower solution of model (58), in the sense that if $u(0, x) \geq a v_{-}(\mu, x-\ell)$, then $u(t, x) \geq a v_{-}(\mu, x-\ell+\psi(\mu) t)$ for all $t>0$.

The proof of this lemma is similar to the previous Lemma IV.8. When $\mu \in$ $\left[0, \mu_{1}\right]$, we have that

$$
r(x-c s)-d \mu^{2} \geq r\left(\ell-\frac{\pi}{\gamma}-\psi\left(\mu_{1}\right) s-c s\right)-d \mu_{1}^{2}
$$




$$
\begin{aligned}
& =r\left(\ell-\frac{\pi}{\gamma}+(c+\epsilon) s-c s\right)-d \mu_{1}^{2} \\
& \geq r(\ell)-d \mu_{1}^{2} \\
& \geq \frac{\left(c^{*}(\infty)-\epsilon\right)^{2}}{4 d}-\frac{(c+\epsilon)^{2}}{4 d}>0 ;
\end{aligned}
$$

and when $\mu \in\left[0, \mu_{2}\right]$, we have that

$$
\begin{aligned}
r(x-c s)-d \mu^{2} & \geq r\left(\ell+\psi\left(\mu_{2}\right) s-c s\right)-d \mu_{2}^{2} \\
& =r\left(\ell+\left(c^{*}(\infty)-\epsilon\right) s-c s\right)-d \mu_{2}^{2} \\
& \geq r(\ell)-d \mu_{2}^{2} \\
& \geq \frac{\left(c^{*}(\infty)-\epsilon\right)^{2}}{4 d}-\frac{\left(c^{*}(\infty)-2 \epsilon\right)^{2}}{4 d}>0 .
\end{aligned}
$$

For $\mu \in\left[\mu_{4}, \mu_{3}\right]$, we have that $r(x-c s)>r(-\infty)$. We omit the details of the proof here.

Lemma IV.10 Assume that Hypothesis IV.1 is satisfied and $c^{*}(-\infty) \leq-c<c^{*}(\infty)$. Assume also $u(t, x)$ is a solution of model (58) with $0<u(0, x) \leq r(\infty)$ for all $x$. For any positive $\epsilon$, there exists $\ell$ such that $c^{*}(\ell)=c^{*}(\infty)-\epsilon$, and $\gamma>0$ such that $c^{*}(\ell)-c_{\gamma}^{*}(\ell) \leq \epsilon$. Let $\psi\left(\mu_{1}\right)=-c-\epsilon$, and $\psi\left(\mu_{2}\right)=c_{\gamma}^{*}(\ell)-\epsilon$. Then for $\mu \in\left[0, \mu_{2}\right]$, and $0<a \leq \epsilon / \mu_{2}$, if $u(0, x) \geq a v(\mu, x-\ell)$, then $u(t, x) \geq a v(\mu, x-\ell-\psi(\mu) t)$ for all $t>0$; for $\mu \in\left[0, \mu_{1}\right]$ and $0<a \leq \epsilon / \mu_{1}$, if $u(0, x) \geq a v_{-}(\mu, x-\ell)$, then $u(t, x) \geq a v_{-}(\mu, x-\ell+\psi(\mu) t)$ for all $t>0$.

Lemma IV.11 Assume that Hypothesis IV.1 is satisfied and $-c \geq c^{*}(\infty)$. Assume also $u(t, x)$ is a solution of model (58) with $0<u(0, x) \leq r(\infty)$ for all $x$. For any positive $\epsilon$, there exists $\ell$ such that $c^{*}(\ell)=c^{*}(\infty)-\epsilon$, and $\gamma>0$ such that $c^{*}(\ell)-c_{\gamma}^{*}(\ell) \leq$ $\epsilon$. Let $\psi\left(\mu_{1}\right)=\psi\left(\mu_{2}\right)=c_{\gamma}^{*}(\ell)-\epsilon$. Then for $\mu \in\left[0, \mu_{2}\right]$, and $0<a \leq \epsilon / \mu_{2}$, if $u(0, x) \geq a v(\mu, x-\ell)$, then $u(t, x) \geq a v(\mu, x-\ell-\psi(\mu) t)$ for all $t>0 ;$ for $\mu \in\left[0, \mu_{1}\right]$ and $0<a \leq \epsilon / \mu_{1}$, if $u(0, x) \geq a v_{-}(\mu, x-\ell)$, then $u(t, x) \geq a v_{-}(\mu, x-\ell+\psi(\mu) t)$ for all $t>0$.

The following lemma shows that functions $w_{l}\left(\alpha, \mu_{1}, \mu_{2} ; t, x\right), w_{r}\left(\alpha, \mu_{1}, \mu_{2} ; t, x\right)$ and $w\left(\alpha, \mu_{1}, \mu_{2} ; t, x\right)$ can translate to the lower solution of system (58) under appropriate conditions. 
Lemma IV.12 Assume that Hypotheses IV.1 is satisfied and $u(t, x)$ is a solution of model (58) with $0<u(0, x) \leq r(\infty)$ for all $x$. Then for any small positive number $\epsilon$, there exist positive numbers $\alpha, \mu_{1}, \mu_{2}, t_{0}$, and $\ell$ satisfying $c^{*}(\ell)=c^{*}(\infty)-\epsilon$. Let $\gamma>0$ such that $c^{*}(\ell)-c_{\gamma}^{*}(\ell) \leq \epsilon$. Then for $t>t_{0}$

(a) If $c>c^{*}(\infty), u(t, x) \geq w\left(\alpha, \mu_{1}, \mu_{2} ; t-t_{0}, x\right)$ with $\psi\left(\mu_{1}\right)=\psi\left(\mu_{2}\right)=c^{*}(-\infty)-\epsilon$;

(b) If $0<c<c^{*}(\infty), u(t, x) \geq w_{r}\left(\alpha, \mu_{1}, \mu_{2} ; t-t_{0}, x\right)$ with $\psi\left(\mu_{1}\right)=c+\epsilon$ and $\psi\left(\mu_{2}\right)=c^{*}(\infty)-\epsilon$; and, $u(t, x) \geq w\left(\alpha, \mu_{1}^{\prime}, \mu_{2}^{\prime} ; t-t_{0}, x\right)$ with $\psi\left(\mu_{1}^{\prime}\right)=\psi\left(\mu_{2}^{\prime}\right)=$ $c^{*}(-\infty)-\epsilon$;

(c) If $c^{*}(-\infty)>-c \geq 0, u(t, x) \geq w\left(\alpha, \mu_{1}, \mu_{2} ; t-t_{0}, x\right)$ with $\psi\left(\mu_{1}\right)=-c-\epsilon$ and $\psi\left(\mu_{2}\right)=c^{*}(\infty)-\epsilon$; and, $u(t, x) \geq w_{l}\left(\alpha, \mu_{1}^{\prime}, \mu_{2}^{\prime} ; t-t_{0}, x\right)$ with $\psi\left(\mu_{1}^{\prime}\right)=c^{*}(-\infty)-\epsilon$ and $\psi\left(\mu_{2}^{\prime}\right)=-c+\epsilon$;

(d) If $c^{*}(-\infty) \leq-c<c^{*}(\infty), u(t, x) \geq w\left(\alpha, \mu_{1}, \mu_{2} ; t-t_{0}, x\right)$ with $\psi\left(\mu_{1}\right)=-c-\epsilon$ and $\psi\left(\mu_{2}\right)=c^{*}(\infty)-\epsilon$;

(e) If $-c \geq c^{*}(\infty), u(t, x) \geq w\left(\alpha, \mu_{1}, \mu_{2} ; t-t_{0}, x\right)$ with $\psi\left(\mu_{1}\right)=\psi\left(\mu_{2}\right)=c^{*}(\infty)-\epsilon$.

The proof of this lemma can be done by constructing a family of lower solutions of model (58) as in the proof of Lemma II.11 and the proof for Lemma 5.2 in Hu and Li (2015). We omit the details. 


\section{Spreading Speed}

Similar to Theorem II.1, we show that when $c$ is large, the species persists in a lattice space and spreads to both right and left at the asymptotic spreading speed $c^{*}(-\infty)$ in the following theorem.

Theorem IV.1 Assume that Hypothesis IV.1 is satisfied and $c^{*}(\infty) \leq c$. Assume also $u(t, x)$ is a solution of model (58) with $0<u(0, x) \leq r(\infty)$ for all $x$.

(a) Let $\mu^{+}=\mu^{*}(\infty)=\sqrt{\frac{r(\infty)}{d}}, \mu^{-}=\mu^{*}(-\infty)=\sqrt{\frac{r(-\infty)}{d}}$ and $\underline{c}=\frac{\mu^{+} c^{*}(\infty)-\mu^{-} c^{*}(-\infty)}{\mu^{+}-\mu^{-}}$. If $u(0, x)$ is zero for all sufficiently large $x, 0 \leq u(0, x) \leq r(\infty)$, and $c>\underline{c}$, then for any positive $\epsilon$,

$$
\lim _{t \rightarrow+\infty}\left[\sup _{x \geq t\left(c^{*}(-\infty)+\epsilon\right)} u(t, x)\right]=0
$$

(b) If $u(0, x)$ is zero for all sufficiently negative $x$, and $0 \leq u(0, x) \leq r(\infty)$, then for any positive $\epsilon$

$$
\lim _{t \rightarrow+\infty}\left[\sup _{x \leq-t\left(c^{*}(-\infty)+\epsilon\right)} u(t, x)\right]=0 .
$$

(c) If $u(0, x)>0$ on a closed interval and $0 \leq u(0, x) \leq r(\infty)$, then for every $\epsilon>0$

$$
\lim _{t \rightarrow+\infty}\left[\inf _{-t\left(c^{*}(-\infty)-\epsilon\right) \leq x \leq t\left(c^{*}(-\infty)-\epsilon\right)} u(t, x)\right]=r(-\infty)
$$

Remark IV.1 Biologically, this theorem implies that, if the species spreading speed in the high quality environment is not sufficient to keep pace with the rate of climate change, then the species effectively only exists in the low quality environment. Since the species is spreading only in the low quality environment, its leftward and rightward spreading speeds are that of the low quality environment $c^{*}(-\infty)$. This theorem does not include the case of $c^{*}(\infty)<c \leq \underline{c}$.

Proof. We first prove statement (a). From statement (a) of Lemma IV.5, we have that for any small positive number $\epsilon$, there exist $A_{1}>0$, such that

$$
u(t, x) \leq u_{1}(t, x)=A_{1} e^{-\mu_{1}\left(x-\left(c^{*}(\infty)+\epsilon\right) t\right)},
$$


where $\mu_{1}$ is the smallest solution of $\phi(\infty ; \mu)=c^{*}(\infty)+\epsilon$. Additionally, from the formula (66), for the given $\epsilon$, there exists $\delta>0$ and $0<\mu_{2}<\mu^{*}(-\infty)$, such that

$$
\mu_{2}\left(c^{*}(-\infty)+\epsilon\right)=2 D\left(e^{\mu_{2}}-2+e^{-\mu_{2}}\right)+r(-\infty)+\delta .
$$

We assume that

$$
u_{2}(t, x)=A_{2} e^{-\mu_{2}\left(x-\left(c^{*}(-\infty)+\epsilon\right) t\right)},
$$

and

$$
S=\frac{\ln A_{1}-\ln A_{2}}{\mu_{1}-\mu_{2}} .
$$

It is straightforward to prove that $u_{2}(t, x)$ is the solution of the following model:

$$
\frac{\partial u}{\partial t}=D[u(t, x+1)-2 u(t, x)+u(t, x-1)]+(r(-\infty)+\delta) u, \quad x \in \mathrm{H}, t>0,
$$

so that $u_{2}(t, x)$ satisfies the following integral equation:

$$
\begin{aligned}
u_{2}(t, x)= & \sum_{m=-\infty}^{+\infty} e^{-(\rho+2 D) t} I_{m}(2 D t) A_{2} e^{-\mu_{2}(x-m)}+ \\
& \int_{0}^{t} \sum_{m=-\infty}^{+\infty} e^{-(\rho+2 D) \tau} I_{m}(2 D \tau) A_{2} e^{-\mu_{2}\left(x-m-\left(c^{*}(-\infty)+\epsilon\right)(t-\tau)\right)}(\rho+r(-\infty)+\delta) d \tau .
\end{aligned}
$$

For any $\epsilon_{1}>0$ sufficiently small, we can choose $\mu_{1}$ and $\mu_{2}$ satisfying that $\mu^{+} \geq \mu_{1}>\mu^{+}-\epsilon_{1}$ and $\mu^{-} \geq \mu_{2}>\mu^{+}-\epsilon_{1}$. So when $\epsilon_{1} \rightarrow 0$, we have that

$$
0<\frac{\mu_{1} c^{*}(\infty)-\mu_{2} c^{*}(-\infty)}{\mu_{1}-\mu_{2}} \leq \underline{c}+\epsilon .
$$

Therefore, when $c>\underline{c}+3 \epsilon$ and $x>(c-\epsilon) t+S$, we have that

$$
\begin{aligned}
\frac{u_{2}(t, x)}{u_{1}(t, x)} & =\frac{A_{2}}{A_{1}} e^{\mu_{1}\left(x-\left(c^{*}(\infty)+\epsilon\right) t\right)-\mu_{2}\left(x-\left(c^{*}(-\infty)+\epsilon\right) t\right)} \\
& =\frac{A_{2}}{A_{1}} e^{\left(\mu_{1}-\mu_{2}\right)\left[x-\left(\frac{\mu_{1} c^{*}(\infty)-\mu_{2} c^{*}(-\infty)}{\mu_{1}-\mu_{2}}+\epsilon\right) t\right]} \\
& \geq \frac{A_{2}}{A_{1}} e^{\left(\mu_{1}-\mu_{2}\right)[x-(\underline{c}+2 \epsilon) t]} \\
& \geq \frac{A_{2}}{A_{1}} e^{\left(\mu_{1}-\mu_{2}\right)(S+(c-\epsilon) t-(c-\epsilon) t)} \\
& \geq \frac{A_{2}}{A_{1}} e^{\left(\mu_{1}-\mu_{2}\right) S}=1
\end{aligned}
$$


It follows that, when $x>(c-\epsilon) t+S, u(t, x) \leq u_{1}(t, x) \leq u_{2}(t, x)$.

On the other hand, when $x \leq x_{0}+c t-b$, where $x_{0}$ satisfies that for any $x<x_{0}, r(x)<r(-\infty)+\delta$. Assume $u(0, x) \leq u_{2}(0, x)=A_{2} e^{-\mu_{2} x}$ and $b$ is introduced in Lemma IV.6 with $\mu=\mu_{2}$. The sequence $u^{(n)}(t, x)$ is defined in the formula (62) with $u^{(0)}(t, x)=0$. Obviously, $u^{(0)}(t, x)=0$ is a lower solution of system (58) and $u(t, x)=\lim _{n \rightarrow \infty} u^{(n)}(t, x)$. We have that

$$
\begin{aligned}
u^{(1)}(t, x) & =Q\left[u^{(0)}\right](t, x)=\sum_{m=-\infty}^{+\infty} e^{-(\rho+2 D) t} I_{m}(2 D t) A_{2} e^{-\mu_{2}(x-m)} \\
& =A_{2} e^{-\mu_{2} x-(\rho+2 D) t}\left[\sum_{m=0}^{+\infty} I_{m}\left(2 D e^{\mu} \tau\right)+\sum_{m=1}^{\infty} I_{m}(2 D \tau)\right] \\
& =A_{2} e^{-\mu_{2} x-(\rho+2 D) t} e^{2 D e^{\mu_{2} t}} \\
& =u_{2}(t, x) e^{-\left(\rho+2 D+c^{*}(-\infty)+\epsilon-2 D e^{\mu_{2}}\right) t} \leq u_{2}(t, x)
\end{aligned}
$$

for $\rho$ sufficiently large. We compute that

$$
\begin{aligned}
Q\left[u_{2}\right](t, x)= & \sum_{m=-\infty}^{+\infty} e^{-(\rho+2 D) t} I_{m}(2 D t) u_{0}(x+m)+\int_{0}^{t} \sum_{m=-\infty}^{+\infty} e^{-(\rho+2 D)(t-\tau)} \\
& \cdot I_{m}(2 D(t-\tau)) u_{2}(\tau, x+m)\left[\rho+r(x+m-c \tau)-u_{2}(\tau, x+m)\right] d \tau \\
= & \sum_{m=-\infty}^{+\infty} e^{-(\rho+2 D) t} I_{m}(2 D t) u_{0}(x-m)+\int_{0}^{t} \sum_{m=-\infty}^{+\infty} e^{-(\rho+2 D) \tau} \\
& \cdot I_{m}(2 D \tau) u_{2}(t-\tau, x-m)\left[\rho+r(x-m-c(t-\tau))-u_{2}(t-\tau, x-m)\right] d \tau \\
\leq & \sum_{m=-\infty}^{+\infty} e^{-(\rho+2 D) t} I_{m}(2 D t) A_{2} e^{-\mu_{2}(x-m)}+\int_{0}^{t} \sum_{m=-\infty}^{+\infty} e^{-(\rho+2 D) \tau} \\
& \cdot I_{m}(2 D \tau) A_{2} e^{-\mu_{2}\left(x-m-\left(c^{*}(-\infty)+\epsilon\right)(t-\tau)\right)}[\rho+r(x-m-c(t-\tau))] d \tau \\
\leq & \sum_{m=-\infty}^{+\infty} e^{-(\rho+2 D) t} I_{m}(2 D t) A_{2} e^{-\mu_{2}(x-m)}+\left(1+S_{0} \epsilon_{1}\right) \int_{0}^{t} \sum_{m=c \tau-b}^{+\infty} e^{-(\rho+2 D) \tau} \\
& \cdot I_{m}(2 D \tau) A_{2} e^{-\mu_{2}\left(x-m-\left(c^{*}(-\infty)+\epsilon\right)(t-\tau)\right)}[\rho+r(x-m-c(t-\tau))] d \tau \\
\leq & \sum_{m=-\infty}^{+\infty} e^{-(\rho+2 D) t} I_{m}(2 D t) A_{2} e^{-\mu_{2}(x-m)}+\left(1+S_{0} \epsilon_{1}\right)(\rho+r(-\infty)+\delta) \\
& \cdot \int_{0}^{t} \sum_{m=c \tau-b}^{+\infty} e^{-(\rho+2 D) \tau} I_{m}(2 D \tau) A_{2} e^{-\mu_{2}\left(x-m-\left(c^{*}(-\infty)+\epsilon\right)(t-\tau)\right)} d \tau .
\end{aligned}
$$

Since $\epsilon_{1}$ is an arbitrary small number, (78) and (77) show that $Q\left[u_{2}\right](t, x) \leq u_{2}(t, x)$. By induction, $u^{(n)}(t, x) \leq u_{2}(t, x)$. Since $u^{(n)}(t, x)$ converges to $u(t, x)$, so $u(t, x) \leq$ $u_{2}(t, x)$ for any $x \leq x_{0}+c t-b$. 
Let $t$ satisfies $t>T_{\epsilon}=\frac{\left(S+b-x_{0}\right)}{\epsilon}$, then $(c-\epsilon) t+S \leq x_{0}+c t-b$, so we have that $u(t, x) \leq u_{2}(t, x)$ for any $\mathrm{x}$ and $t$ sufficiently large. Therefore, the statement (a) is true.

Now we consider the statement (b). From the statement (b) in Lemma IV.5, for any $\epsilon>0$, there exist positive numbers $B$ and $\mu_{1}$ such that

$$
0 \leq u(t, x) \leq B e^{\mu_{1}\left(x+\left(c^{*}(-\infty)+\frac{\epsilon}{2}\right) t\right)}
$$

It follows that

$$
\begin{aligned}
0 \leq \lim _{t \rightarrow+\infty}\left[\sup _{x \leq-t\left(c^{*}(-\infty)+\epsilon\right)} u(t, x)\right] & \leq \lim _{t \rightarrow+\infty}\left[\sup _{x \leq-t\left(c^{*}(-\infty)+\epsilon\right)} B e^{\mu_{1}\left(x+\left(c^{*}(-\infty)+\frac{\epsilon}{2}\right) t\right)}\right] \\
& \leq \lim _{t \rightarrow+\infty}\left[B e^{\mu_{1}\left(-t\left(c^{*}(-\infty)+\epsilon\right)+\left(c^{*}(-\infty)+\frac{\epsilon}{2}\right) t\right)}\right] \\
& \leq \lim _{t \rightarrow+\infty}\left[B e^{-\mu_{1} \frac{\epsilon}{2} t}\right]=0 .
\end{aligned}
$$

Therefore,

$$
\lim _{t \rightarrow+\infty}\left[\sup _{x \leq-t\left(c^{*}(-\infty)+\epsilon\right)} u(t, x)\right]=0 .
$$

Finally, we prove statement (c). By Lemma IV.4, for any $\epsilon>0$, there exist sufficiently large $T_{1}>0$ and $M>0$, such that, when $t>T_{1}$ and $x \leq-M+c t$,

$$
u(t, x) \leq r(-\infty)+\epsilon .
$$

Form statement (a) of Lemma IV.5, we have that if $u(0, x)$ is zero for all sufficiently large $x$, and $0 \leq u(0, x) \leq r(\infty)$, then for any positive $\epsilon$ there exist positive numbers $A$ and $\mu_{\epsilon}$ such that

$$
u(t, x) \leq A e^{-\mu_{\epsilon}\left(x-\left(c^{*}(\infty)+\frac{\epsilon}{2}\right) t\right)} .
$$

When $x \geq\left(c^{*}(\infty)+\epsilon\right) t$, we have that

$$
u(t, x) \leq A e^{-\mu_{\epsilon} \frac{\epsilon}{2} t}
$$

It follows that, for the given $\epsilon$, there exists a sufficiently large $T_{2}$, such that

$$
u(t, x)<r(-\infty)+\epsilon \text { for } t \geq T_{2} \text { and } x \geq\left(c^{*}(\infty)+\epsilon\right) t
$$

Since $c^{*}(\infty)<c$, there exists $T_{3}>\max \left\{T_{1}, T_{2}\right\}$ such that for $t>T_{3},-M+c t>$ $\left(c^{*}(\infty)+\epsilon\right) t$. Therefore, when $t>T_{3}$, we have that $u(t, x) \leq r(-\infty)+\epsilon$ for any $x$. 
On the other hand, from Lemma IV.12, choose $0<\epsilon<\min \left\{r(\infty), \frac{c^{*}(\infty)-c}{5}\right\}$ and $t_{0}>0, \alpha>0$ and $\gamma>0$ sufficiently small such that $u\left(t_{0}, x\right) \geq \omega\left(t_{0}\right)$. We have that for any $t>t_{0}, x \in \mathrm{H}$,

$$
u(t, x) \geq w\left(\alpha, \mu_{1}, \mu_{2} ; t-t_{0}, x\right)
$$

where $\psi\left(\mu_{1}\right)=\psi\left(\mu_{2}\right)=c^{*}(-\infty)-\epsilon / 2$.

By using Corollary IV.1, for the given $\epsilon>0$, there is $M>0$ such that

$$
\sum_{|m| \leq \max \{M, \sqrt{2 D M \tau}\}} e^{-2 D \tau} I_{m}(2 D \tau) \geq 1-\epsilon,
$$

for any $\tau>0$. If $u\left(t_{0}, x\right)$ is assumed to be the initial value for the model (58), then for sufficiently large $t_{1} \geq t_{0}$, the solution $u(t, x)$ satisfies the integral equation for $t>t_{1}$

$$
\begin{aligned}
u(t, x)= & \sum_{m=-\infty}^{+\infty} e^{-(\rho+2 D)\left(t-t_{1}\right)} I_{m}\left(2 D\left(t-t_{1}\right)\right) u\left(t_{1}, x+m\right) \\
& +\int_{t_{1}}^{t} \sum_{m=-\infty}^{+\infty} e^{-(\rho+2 D)(t-\tau)} I_{m}(2 D(t-\tau)) \\
& \cdot u(\tau, x+m)[\rho+r(x+m-c \tau)-u(\tau, x+m)] d \tau .
\end{aligned}
$$

When $t>t_{1}, x$ satisfies

$$
\begin{aligned}
& \ell-\sigma\left(\mu_{1}\right)-\psi\left(\mu_{1}, \gamma\right)\left(t-t_{0}\right)+\max \left\{M, \sqrt{2 D M\left(t-t_{1}\right)}\right\} \leq x \\
& \leq \ell+3 \pi / \gamma+\sigma\left(\mu_{2}\right)+\psi\left(\mu_{2}, \gamma\right)\left(t-t_{0}\right)-\max \left\{M, \sqrt{2 D M\left(t-t_{1}\right)}\right\}
\end{aligned}
$$

and $m$ satisfies

$$
-\max \left\{M, \sqrt{2 D M\left(t-t_{1}\right)}\right\} \leq m \leq \max \left\{M, \sqrt{2 D M\left(t-t_{1}\right)}\right\}
$$

we have that

$$
\ell-\sigma\left(\mu_{1}\right)-\psi\left(\mu_{1}, \gamma\right)\left(t-t_{0}\right) \leq x+m \leq \ell+3 \pi / \gamma+\sigma\left(\mu_{2}\right)+\psi\left(\mu_{2}, \gamma\right)\left(t-t_{0}\right)
$$

By direct calculation, the linear part of equation (79) satisfies that

$$
\begin{aligned}
& \sum_{m=-\infty}^{+\infty} e^{-(\rho+2 D)\left(t-t_{1}\right)} I_{m}\left(2 D\left(t-t_{1}\right)\right) u\left(t_{1}, x+m\right) \\
\geq & \sum_{|m| \leq \max \left\{M, \sqrt{2 D M\left(t-t_{1}\right)}\right\}} e^{-(\rho+2 D)\left(t-t_{1}\right)} I_{m}\left(2 D\left(t-t_{1}\right)\right) \alpha \\
\geq & (1-\epsilon) \alpha e^{-\rho\left(t-t_{1}\right)} .
\end{aligned}
$$


In the nonlinear part, we have that

$$
\begin{aligned}
& \sum_{m=-\infty}^{+\infty} e^{-(\rho+2 D)(t-\tau)} I_{m}(2 D(t-\tau)) u(\tau, x+m)[\rho+r(x+m-c \tau)-u(\tau, x+m)] \\
\geq & (1-\epsilon) \alpha[\rho+r(-\infty)-\epsilon-\alpha] e^{-\rho(t-\tau)} .
\end{aligned}
$$

It follows from (79)-(84) that for $t \geq t_{0}$ and $x$ satisfying (80)

$$
u(t, x) \geq \tilde{u}^{(1)}(t)
$$

where

$$
\tilde{u}^{(1)}(t)=(1-\epsilon) \alpha e^{-\rho\left(t-t_{1}\right)}+\int_{t_{1}}^{t} e^{-\rho(t-\tau)}(1-\epsilon) \alpha[\rho+r(-\infty)-\epsilon-\alpha] d \tau .
$$

It follows from this, (79) and induction that for $t \geq t_{1}$ and $x$ satisfying (80),

$$
u(t, x) \geq \tilde{u}^{(n)}(t)
$$

where $\tilde{u}^{(n)}(t)$ satisfies

$$
\begin{aligned}
\tilde{u}^{(n+1)}(t) & =(1-\epsilon) \alpha e^{-\rho\left(t-t_{0}\right)} \\
& +\int_{t_{0}}^{t} e^{-\rho(t-\tau)}(1-\epsilon) \tilde{u}^{(n)}(\tau)\left[\rho+r(-\infty)-\epsilon-\frac{1}{1-\epsilon} \tilde{u}^{(n)}(\tau)\right] d \tau \\
& =a_{n}+b_{n}(t) e^{-\rho\left(t-t_{0}\right)}
\end{aligned}
$$

In this formula,

$$
\begin{gathered}
a_{n}=\frac{(1-\epsilon) a_{n-1}\left(\rho+r(-\infty)-\epsilon-a_{n-1}\right)}{\rho}, \\
a_{1}=\frac{(1-\epsilon) \alpha[\rho+r(-\infty)-\epsilon-\alpha]}{\rho},
\end{gathered}
$$

and $b_{n}(t)$ is the sum of polynomials, and products of polynomials and exponential functions in the form of $e^{-j \rho\left(t-t_{0}\right)}$ with $j$ a positive integer.

Since $\lim _{t \rightarrow \infty} \tilde{u}^{(n)}(t)=a_{n}$ and $\lim _{n \rightarrow \infty} a_{n}=r(-\infty)-\epsilon-\epsilon \rho /(1-\epsilon)$, there exists a positive integer $N$ sufficiently large, for $t>t_{1}$

$$
\tilde{u}^{(N)}(t) \geq r(-\infty)-\epsilon-\epsilon \rho /(1-\epsilon)
$$

Choose $t_{2}>t_{1}$ sufficiently large such that when $t>t_{2}$,

$$
\begin{gathered}
\ell-\sigma\left(\mu_{1}\right)-\psi\left(\mu_{1}, \gamma\right)\left(t-t_{1}\right)+N \max \left\{M, \sqrt{2 D M\left(t-t_{1}\right)}\right\} \\
\leq \ell+3 \pi / \gamma+\sigma\left(\mu_{2}\right)+\psi\left(\mu_{2}, \gamma\right)\left(t-t_{1}\right)-N \max \left\{M, \sqrt{2 D M\left(t-t_{1}\right)}\right\},
\end{gathered}
$$


and also there exists $t_{3}>t_{2}$ such that when $t>t_{3}$

$$
\begin{aligned}
& \ell-\sigma\left(\mu_{1}\right)-\psi\left(\mu_{1}, \gamma\right)\left(t-t_{1}\right)+N \max \left\{M, \sqrt{2 D M\left(t-t_{1}\right)}\right\} \\
\leq & -t\left(c^{*}(-\infty)-\epsilon\right) \leq t\left(c^{*}(-\infty)-\epsilon\right) \\
\leq & \ell+3 \pi / \gamma+\sigma\left(\mu_{2}\right)+\psi\left(\mu_{2}, \gamma\right)\left(t-t_{1}\right)-N \max \left\{M, \sqrt{2 D M\left(t-t_{1}\right)}\right\} .
\end{aligned}
$$

Equations (85), (86) and (87) show that when $t>t_{3}$ and $-t\left(c^{*}(-\infty)-\epsilon\right) \leq x \leq$ $t\left(c^{*}(-\infty)-\epsilon\right)$

$$
\lim _{t \rightarrow \infty}\left[\inf _{-t\left(c^{*}(-\infty)-\epsilon\right) \leq x \leq t\left(c^{*}(-\infty)-\epsilon\right)} u(t, x)\right] \geq(1-\epsilon)(r(\infty)-\epsilon) .
$$

Since $\epsilon$ is arbitrary and $u(t, x) \leq r(\infty)$ for all $x$ and $t$, it follows that

$$
\lim _{t \rightarrow+\infty}\left[\inf _{-t\left(c^{*}(-\infty)-\epsilon\right) \leq x \leq t\left(c^{*}(-\infty)-\epsilon\right)} u(t, x)\right]=r(-\infty) .
$$

The proof is complete.

The following theorem shows that if $c^{*}(\infty)>c \geq 0$, then the species persists in a lattice space and spreads to the right at the asymptotic spreading speed $c^{*}(\infty)$ and to the left at the asymptotic spreading speed $c^{*}(-\infty)$, forming a two-layer wave.

Theorem IV.2 Assume that Hypothesis IV.1 is satisfied and $c^{*}(\infty)>c \geq 0$. Assume also $u(t, x)$ is a solution of model (58) with $0<u(0, x) \leq r(\infty)$ for all $x$.

(a) If $u(0, x)$ is zero for all sufficiently large $x$, and $0 \leq u(0, x) \leq r(\infty)$, then for any positive $\epsilon$

$$
\lim _{t \rightarrow+\infty}\left[\sup _{x \geq t\left(c^{*}(\infty)+\epsilon\right)} u(t, x)\right]=0 .
$$

(b) If $0 \leq u(0, x) \leq r(\infty), u(0, x)>0$ on a closed interval, and $u(0, x)$ is zero for all sufficiently large $x$, then for every $\epsilon$ with $0<\epsilon<\frac{\left(c^{*}(\infty)-c\right)}{2}$

$$
\lim _{t \rightarrow+\infty}\left[\sup _{t(c+\epsilon) \leq x \leq t\left(c^{*}(\infty)-\epsilon\right)}|r(\infty)-u(t, x)|\right]=0 .
$$

(c) If $u(0, x)$ is zero for all sufficiently negative $x$, and $0 \leq u(0, x) \leq r(\infty)$, then for any positive $\epsilon$

$$
\lim _{t \rightarrow+\infty}\left[\sup _{x \leq-t\left(c^{*}(-\infty)+\epsilon\right)} u(t, x)\right]=0 .
$$


(d) If $u(0, x)>0$ on a closed interval and $0 \leq u(0, x) \leq r(\infty)$, then for every $\epsilon>0$

$$
\lim _{t \rightarrow+\infty}\left[\inf _{-t\left(c^{*}(-\infty)-\epsilon\right) \leq x \leq \min \left\{t(c-\epsilon), t\left(c^{*}(-\infty)-\epsilon\right)\right\}} u(t, x)\right]=r(-\infty)
$$

Remark IV.2 Here we mention some of its biological implications in the theorem. Essentially if the species' spreading speed in the high quality environment is sufficient to keep ahead of the rightward moving favorable habitat boundary, then the species will continue to expand ahead in the high quality environment at the speed $c^{*}(\infty)$. Similarly the species will continue to expand leftward into the low quality environment at the speed associated with the low quality environment, $c^{*}(-\infty)$. Statement (b) states that for regions behind the forward invasion front but sufficiently in front of the favorable habitat boundary, the population will essentially reach the high quality habitat equilibrium, $r(\infty)$. Similarly (d) states that for a region in front of the rearward invasion but sufficiently behind the favorable habitat boundary the population will essentially achieve the equilibrium value of the poor quality habitat, $r(-\infty)$.

Proof. We prove statement (a). From (a) of Lemma IV.5, we have that for any positive $\epsilon$, there exist positive numbers $A$ and $\mu_{\epsilon}$, such that

$$
u(t, x) \leq A e^{-\mu_{\epsilon}\left(x-\left(c^{*}(\infty)+\frac{\epsilon}{2}\right) t\right)} .
$$

The trivial solution $u=0$ is a lower solution of model (58), so $u(t, x) \geq 0$ for all $t>0$ and $x$. It follows that

$$
\begin{aligned}
0 \leq \lim _{t \rightarrow+\infty}\left[\sup _{x \geq t\left(c^{*}(\infty)+\epsilon\right)} u(t, x)\right] & \leq \lim _{t \rightarrow+\infty}\left[\sup _{x \geq t\left(c^{*}(\infty)+\epsilon\right)} A e^{-\mu_{\epsilon}\left(x-\left(c^{*}(\infty)+\frac{\epsilon}{2}\right) t\right)}\right] \\
& =\lim _{t \rightarrow+\infty}\left[A e^{-\mu_{\epsilon}\left(t\left(c^{*}(\infty)+\epsilon\right)-\left(c^{*}(\infty)+\frac{\epsilon}{2}\right) t\right)}\right] \\
& =\lim _{t \rightarrow+\infty}\left[A e^{-\mu_{\epsilon} \frac{\epsilon}{2} t}\right]=0 .
\end{aligned}
$$

Therefore

$$
\lim _{t \rightarrow+\infty}\left[\sup _{x \geq t\left(c^{*}(\infty)+\epsilon\right)} u(t, x)\right]=0 .
$$

The proof of statement (b) is similar to the one of statement(iii) in Theorem 5.1 of $\mathrm{Hu}$ and $\mathrm{Li}$ (2015) and is omitted. The proof of the statement (c) is identical to the proof of statement (c) in Theorem IV.1, so we omit the proof as well. 
Finally, we prove the statement (d). From the proof of statement (c) in Theorem IV.1, we have that

$$
\lim _{t \rightarrow+\infty}\left[\inf _{-t\left(c^{*}(-\infty)-\epsilon\right) \leq x \leq t\left(c^{*}(-\infty)-\epsilon\right)} u(t, x)\right] \geq r(-\infty)
$$

In other words, for any given $\epsilon>0$, there exist $T_{0}$, such that

$$
u(t, x) \geq r(-\infty), \quad \text { if } t>T_{0}, \text { and }-t\left(c^{*}(-\infty)-\epsilon\right) \leq x \leq t\left(c^{*}(-\infty)-\epsilon\right)
$$

On the other hand, by using Lemma IV.4, for the given $\epsilon>0$, there exists sufficiently large $T>0$ and $M>0$, such that,

$$
u(t, x) \leq r(-\infty)+\epsilon, \quad \text { if } t>T \text {, and } x \leq-M+c t
$$

If $c \leq c^{*}(-\infty)$, it follows from (88) and (89) that when $t>\max \left\{T, T_{0}, \frac{M}{\epsilon}\right\}$ and $-t\left(c^{*}(-\infty)-\epsilon\right) \leq x \leq t(c-\epsilon)$,

$$
r(-\infty) \leq u(t, x) \leq r(-\infty)+\epsilon .
$$

If $c \geq c^{*}(-\infty)$, it follows from (88) and (89) that when $t>\max \left\{T, T_{0}, \frac{M}{c-c^{*}(-\infty)+\epsilon}\right\}$ and $-t\left(c^{*}(-\infty)-\epsilon\right) \leq x \leq t\left(c^{*}(-\infty)-\epsilon\right)$,

$$
r(-\infty) \leq u(t, x) \leq r(-\infty)+\epsilon
$$

Since $\epsilon$ can be any small positive number, we have that

$$
\lim _{t \rightarrow+\infty}\left[\inf _{-t\left(c^{*}(-\infty)-\epsilon\right) \leq x \leq \min \left\{t(c-\epsilon), t\left(c^{*}(-\infty)-\epsilon\right)\right\}} u(t, x)\right]=r(-\infty) .
$$

The proof is complete.

The following theorem will show that when $-c^{*}(-\infty)<c<0$, the species persists in a lattice space and spreads to the right at the asymptotic spreading speed $c^{*}(\infty)$ and to the left at the asymptotic spreading speed $c^{*}(-\infty)$. The solution of model (58) form a two-layer wave.

Theorem IV.3 Assume that Hypothesis IV.1 is satisfied and $-c^{*}(-\infty)<c<0$. Assume also $u(t, x)$ is a solution of model (58) with $0<u(0, x) \leq r(\infty)$ for all $x$. 
(a) If $u(0, x)$ is zero for all sufficiently large $x$, and $0 \leq u(0, x) \leq r(\infty)$, then for any positive $\epsilon$

$$
\lim _{t \rightarrow+\infty}\left[\sup _{x \geq t\left(c^{*}(\infty)+\epsilon\right)} u(t, x)\right]=0 .
$$

(b) If $0 \leq u(0, x) \leq r(\infty), u(0, x)>0$ on a closed interval, and $u(0, x)$ is zero for all sufficiently large $x$, then for every $\epsilon$ with $0<\epsilon<\frac{\left(c^{*}(\infty)-c\right)}{2}$

$$
\lim _{t \rightarrow+\infty}\left[\inf _{-t(-c-\epsilon) \leq x \leq t\left(c^{*}(\infty)-\epsilon\right)} u(t, x)\right]=r(\infty) .
$$

(c) If $u(0, x)$ is zero for all sufficiently negative $x$, and $0 \leq u(0, x) \leq r(\infty)$, then for any positive $\epsilon$

$$
\lim _{t \rightarrow+\infty}\left[\sup _{x \leq-t\left(c^{*}(-\infty)+\epsilon\right)} u(t, x)\right]=0 .
$$

(d) If $u(0, x)>0$ on a closed interval and $0 \leq u(0, x) \leq r(\infty)$, then for every $\epsilon>0$

$$
\lim _{t \rightarrow+\infty}\left[\inf _{\left.-t\left(c^{*}(-\infty)-\epsilon\right) \leq x \leq-t(-c+\epsilon)\right\}} u(t, x)\right]=r(-\infty) .
$$

Remark IV.3 The theorem has some biological meanings. If the species spreading speed in the low quality environment is in excess of the leftward drift of the habitat boundary, then the species will continue to spread leftward at a speed associated with the low quality habitat, $c^{*}(-\infty)$. The rightward expansion will be at the speed associated with the high quality environment, $c^{*}(\infty)$.

Proof. The proofs for statement (a) and statement (c) are the same as those for statement (a) and statement (c) in Theorem IV.2. We omit them here.

Now we prove statement (b). From statement (c) of Lemma IV.12, for any small positive number $\epsilon$, there exist $\alpha, \gamma, \mu_{1}, \mu_{2}$, and a positive number $t_{0}$, such that when $t>t_{0}$,

$$
u(t, x) \geq w\left(\alpha, \mu_{1}, \mu_{2} ; t-t_{0}, x\right),
$$

where $\psi\left(\mu_{1}\right)=-c-\epsilon / 2$ and $\psi\left(\mu_{2}\right)=c^{*}(\infty)-\epsilon / 2$. If $u\left(t_{0}, x\right)$ is assumed to be the initial value for the model $(58)$, then the solution $u(t, x)$ satisfies the integral equation (79). Since when $t>t_{0}, x$ satisfies

$$
\begin{aligned}
& \ell-\sigma\left(\mu_{1}\right)-\psi\left(\mu_{1}, \gamma\right)\left(t-t_{0}\right)+\max \left\{M, \sqrt{2 D M\left(t-t_{1}\right)}\right\} \leq x \\
& \leq \ell+3 \pi / \gamma+\sigma\left(\mu_{2}\right)+\psi\left(\mu_{2}, \gamma\right)\left(t-t_{0}\right)-\max \left\{M, \sqrt{2 D M\left(t-t_{1}\right)}\right\}
\end{aligned}
$$


and $m$ satisfies

$$
-\max \left\{M, \sqrt{2 D M\left(t-t_{1}\right)}\right\} \leq m \leq \max \left\{M, \sqrt{2 D M\left(t-t_{1}\right)}\right\},
$$

we have that

$$
\ell-\sigma\left(\mu_{1}\right)-\psi\left(\mu_{1}, \gamma\right)\left(t-t_{0}\right) \leq x+m \leq \ell+3 \pi / \gamma+\sigma\left(\mu_{2}\right)+\psi\left(\mu_{2}, \gamma\right)\left(t-t_{0}\right)
$$

SO

$$
x-y-c t \geq \ell-\sigma\left(\mu_{1}\right)-\psi\left(\mu_{1}, \gamma\right)\left(t-t_{0}\right)-c t=\ell-\sigma\left(\mu_{1}\right)+\epsilon / 2\left(t-t_{0}\right)-\epsilon / 2 t_{0} \geq \ell .
$$

Similar to the process in the proof of statement (c) of Theorem IV.1, from (90)-(93) we can prove that

$$
\lim _{t \rightarrow \infty}\left[\inf _{-t(-c-\epsilon) \leq x \leq t\left(c^{*}(\infty)-\epsilon\right)} u(t, x)\right] \geq(1-\epsilon)(r(\infty)-\epsilon) .
$$

Since $\epsilon$ is arbitrary small and $u(t, x) \leq r(\infty)$ for all $x$ and $t$, it follows that

$$
\lim _{t \rightarrow+\infty}\left[\inf _{-t(-c-\epsilon) \leq x \leq t\left(c^{*}(\infty)-\epsilon\right)} u(t, x)\right]=r(\infty) .
$$

Finally, we prove statement (d). From statement (c) of Lemma IV.12, for any small positive number $\epsilon$, there exist $\alpha, \gamma, \mu_{1}, \mu_{2}$, and a positive number $t_{0}$, such that when $t>t_{0}$

$$
u(t, x) \geq w_{l}\left(\alpha, \mu_{1}^{\prime}, \mu_{2}^{\prime} ; t-t_{0}, x\right)
$$

where $\psi\left(\mu_{1}^{\prime}\right)=c^{*}(-\infty)-\epsilon$ and $\psi\left(\mu_{2}^{\prime}\right)=-c+\epsilon$. Using the same method to prove the statement (c), we have that

$$
\lim _{t \rightarrow+\infty}\left[\inf _{\left.-t\left(c^{*}(-\infty)-\epsilon\right) \leq x \leq-t(-c+\epsilon)\right\}} u(t, x)\right] \geq r(-\infty) .
$$

Additionally, by using Lemma IV.4, for the given $\epsilon>0$, there exist sufficiently large $T>0$ and $M>0$, such that, when $t>T$ and $x \leq-M+c t, u(t, x) \leq r(-\infty)+\epsilon$. Then when $t>\frac{M}{\epsilon}$, we have that $-t(-c+\epsilon)<-M+c t$, so that $u(t, x) \leq r(-\infty)+\epsilon$ for any $x$. Therefore,

$$
\lim _{t \rightarrow+\infty}\left[\inf _{\left.-t\left(c^{*}(-\infty)-\epsilon\right) \leq x \leq-t(-c+\epsilon)\right\}} u(t, x)\right]=r(-\infty) .
$$

The proof is complete.

The following theorem deals with when the habitat shifts rapidly leftward, $c^{*}(\infty) \geq-c \geq c^{*}(-\infty)$. 
Theorem IV.4 Assume that Hypothesis IV.1 is satisfied and $c^{*}(\infty) \geq-c \geq c^{*}(-\infty)$. Assume also $u(t, x)$ is a solution of model (58) with $0<u(0, x) \leq r(\infty)$ for all $x$.

(a) If $u(0, x)$ is zero for all sufficiently large $x$, and $0 \leq u(0, x) \leq r(\infty)$, then for any positive $\epsilon$

$$
\lim _{t \rightarrow+\infty}\left[\sup _{x \geq t\left(c^{*}(\infty)+\epsilon\right)} u(t, x)\right]=0
$$

(b) If $u(0, x)>0$ on a closed interval and $0 \leq u(0, x) \leq r(\infty)$, then for every $\epsilon$ satisfying $0<\epsilon<\frac{c^{*}(\infty)-\epsilon}{3}$,

$$
\lim _{t \rightarrow+\infty}\left[\inf _{t(c+\epsilon) \leq x \leq t\left(c^{*}(\infty)-\epsilon\right)} u(t, x)\right]=r(\infty) .
$$

(c) If $u(0, x)$ is zero for all sufficiently negative $x$, and $0 \leq u(0, x) \leq r(\infty)$, then for any positive $\epsilon$

$$
\lim _{t \rightarrow+\infty}\left[\sup _{x \leq-t(-c+\epsilon)} u(t, x)\right]=0
$$

Remark IV.4 Biologically if the leftward moving speed of the favorable habitat boundary exceeds the spreading speed in the low quality habitat, but is lower than the spreading speed in the high quality habitat, then the species will effectively only exist in the high quality habitat. Its leftward spreading speed will be the moving speed of habitat boundary, c, and rightward spreading speed will be $c^{*}(\infty)$.

Proof. Statement (a) is obvious, so we omit the details of the proof here. The proof of statement (b) is similar to the proof of statement (c) in Theorem IV.1 with the lower solution defined in statement $(\mathrm{d})$ of Lemma IV.12, which is that when $t>t_{0}$

$$
u(t, x) \geq w\left(\alpha, \mu_{1}, \mu_{2} ; t-t_{0}, x\right),
$$

where $\psi\left(\mu_{1}\right)=-c-\epsilon$ and $\psi\left(\mu_{2}\right)=c^{*}(\infty)-\epsilon$. We omit the details of the proof here.

The proof of statement (c) is similar to the proof of statement (a) in Theorem IV.1, so we omit the details. The proof is complete.

The following theorem deals with when $c^{*}(\infty) \leq-c$, so the habitat shifts extremely rapidly leftward. 
Theorem IV.5 Assume that Hypothesis IV.1 is satisfied and $c^{*}(\infty) \leq-c$. Assume also $u(t, x)$ is a solution of model (58) with $0<u(0, x) \leq r(\infty)$ for all $x$.

(a) If $u(0, x)$ is zero for all sufficiently large $x$, and $0 \leq u(0, x) \leq r(\infty)$, then for any positive $\epsilon$

$$
\lim _{t \rightarrow+\infty}\left[\sup _{x \geq t\left(c^{*}(\infty)+\epsilon\right)} u(t, x)\right]=0
$$

(b) If $u(0, x)>0$ on a closed interval and $0 \leq u(0, x) \leq r(\infty)$, then for every $\epsilon$ satisfying $0<\epsilon<\frac{c^{*}(\infty)-\epsilon}{3}$,

$$
\lim _{t \rightarrow+\infty}\left[\inf _{-t\left(c^{*}(\infty)-\epsilon\right) \leq x \leq t\left(c^{*}(\infty)-\epsilon\right)} u(t, x)\right]=r(\infty) .
$$

(c) If $u(0, x)$ is zero for all sufficiently negative $x$, and $0 \leq u(0, x) \leq r(\infty)$, then for any positive $\epsilon$

$$
\lim _{t \rightarrow+\infty}\left[\sup _{x \leq-t\left(c^{*}(\infty)+\epsilon\right)} u(t, x)\right]=0 .
$$

Remark IV.5 Biologically, if the leftward moving speed of the favorable habitat is extremely large, then the species will effectively only exist in the high quality habitat. The species will persist and spread in both directions in the habitat with spreading speed $c^{*}(\infty)$. The proof of this theorem is similar to theorem II.4 whose lower solution is defined in statement (e) of Lemma II.11. We only provide the theorem here. 


\section{Discussion for Lattice Differential Model Chapter}

In this chapter, we studied the growth and spread of a species associated to a lattice differential model in a shifting habitat. In our study, the region suitable for species growth was assumed to be expanding or contracting, the species was assumed to grow in the whole lattice space, but with different levels of success. Results in this chapter are extensions of the work by $\mathrm{Hu}$ and $\mathrm{Li}$ (2015) in the following sense. In the work by $\mathrm{Hu}$ and $\mathrm{Li}$ (2015), the region suitable for species growth was assumed to be contracting and species can persist at just one end. As our study shows, the persistence and spread of an invasive species have been made more complicated to analyze due to the interplay among the three speeds, $c^{*}(-\infty), c$ and $c^{*}(-\infty)$, and some new wave propagation patterns have been found to exist. We summarize our main results as follows:

- When the favorable habitat for species growth is contracting, the rightward spreading speed is $c^{*}(\infty)$ if the contracting speed $c$ is relatively small and is $c^{*}(-\infty)$ if the contracting speed $c$ is relatively large. In this case, the leftward spreading speed is $c^{*}(-\infty)$.

- When the favorable habitat for species growth is expanding, the rightward spreading speed is $c^{*}(\infty)$, while the leftward spreading speed depends on how fast climate change is occurring relative to the intrinsic rate of spread of invasive species. We showed that the leftward spreading speed is $c^{*}(\infty)$ for relatively rapidly expansion, $|c|$ itself for relatively intermediate expansion, and $c^{*}(-\infty)$ for relatively slow expansion.

- The solution of the lattice differential equations can form a two-layer wave with analytically determined propagation speeds, which was not found in the work of $\mathrm{Hu}$ and $\mathrm{Li}(2015)$. 


\section{CHAPTER V}

\section{DISCUSSION AND FUTURE DIRECTIONS}

\section{Conclusion}

In this dissertation, we established the persistence and spreading dynamics a-

long a shifting habitat gradient of population with reaction-diffusion equations, the Lotka-Volterra competition model and lattice differential equations. The models considered here are extended or modified versions of the basic Fisher's equation. By using these models, we showed the effect of climate changes on the population development of different species. Climate change can result in habitat shifts (e.g., Polovina et al. 2011; Parr et al. 2012), expansions (Ni 2000; Gonzalez et al. 2010; Lynch et al. 2012), and Contractions (Ni 2000; Gonzalez et al. 2010; Parr et al. 2012), causing the favorable habitat to either recede or envelope the established population. We analyzed some important factors for population growth which include population growth rate, resource distribution, speed and direction of habitat shift due to climate change in continuous or discrete space. We also started the discussion of the persistence and spreading dynamics for multiple species. We focused on the competition model with the assumption that their suitable habitats move in opposite directions.

In Chapter II, for a single species we assume the resource quality is low in the negative direction switching to high on the positive direction. The edge between areas with good quality resources and areas with bad quality resources could shift toward the positive(right) direction or the negative(left) direction. When the good quality resource region is shrinking and the resource boundary moves faster than the maximum population expansion rate, i.e. $c>c^{*}(\infty)$, the spreading speed of the species will approach the asymptotic spreading speed $\left(c^{*}(-\infty)\right)$ corresponding to the low 
quality habitat. When the resource boundary moves slower than the maximum population expansion rate, the species will persist and spread in the rightward direction with the speed of $c^{*}(\infty)$ and in the leftward direction with the speed of $c^{*}(-\infty)$.

On the other hand, the suitable habitat can drift in the negative direction as well. When the resource boundary moves slower than the asymptotic spreading speed $\left(c^{*}(-\infty)\right)$ corresponding to the low quality habitat, i.e. $-c<c^{*}(-\infty)$, we find that the spreading speed of the species in the negative direction equals $c^{*}(-\infty)$ and the spreading speed in the positive direction would be $c^{*}(+\infty)$. When the expanding rate of habitat space is greater than the minimum population expansion rate, i.e. $-c>$ $c^{*}(-\infty)$, the spreading speed of the species toward the left equals one of $|c|, c^{*}(-\infty)$, or $c^{*}(\infty)$. These conclusions are also valid for the lattice differential equations, which are discussed in Chapter IV.

In Chapter III, we analyzed the simplest Lotka-Volterra competition model, assuming the respective suitable habitats of species, represented by $u$ and $v$, are drifting away from the established population. We illustrated that, when the drift speed of the habitats exceeds the respective species' spreading speeds, both of them will become extinct. When the drift speed for the habitats of both species is slower than their respective maximum population expansion rate, each species will survive and spread into their respective habitats. Additionally, if one species' population expansion rate exceeds the habitat drift rate, this species will persist and spread in its suitable habitat. The discussions in this chapter are only the rudimentary work for multi-species models. There are still various questions to be considered in future work. We provided examples of these at the end of Chapter III.

\section{Discussion}

Global change has become more and more rapid in recent years, which is causing substantial changes for habitat gradients of many species. These gradients includes abiotic conditions, the availability of food resources, the density or diversity of interacting species, or other attributes. One of most common examples of gradients is the temperature gradient, which has moved both poleward and toward higher 
elevations. The temperature gradient might result in range expansions, range contractions, and range shifts of many species, so many of them will need to migrate to remain in a suitable habitat (Greenstein and Pandolfi 2008; Hodkinson 2005; Keleher and Rahel 1996).

In this dissertation, we discussed the role of expanding/contracting habitat gradients on single species populations in continuous space by using the reactiondiffusion modeling framework in Chapter II and in discrete space by using the lattice differential modeling framework in Chapter IV. We also analyzed multi-species populations by using the Lotka-Volterra competition model in Chapter III. Two scenarios were considered. First, the favorable habitat of the species is contracting so the species is faced with encroachment of a range boundary (i.e., range contraction). Second, the favorable habitat of the species is expanding so the species is faced by range expansion.

From our results, for the first scenario, persistence of habitats and species requires that the species expand at a rate faster than the boundary recession rate. If the species can expand at a rate faster than climate change, the species could continue to spread even though they lose their habitat at one boundary. One good example for species facing range encroachment is Scotch Broom (Cytisus Scoparius). The natural spreading speed of Scotch Broom was estimated to be $0.5 \mathrm{~m} / \mathrm{yr}$ (Downey and Smith 2000; Neubert and Parker 2004), and the velocity of climate change was demonstrated to be $0.08-1.26 \mathrm{~km} / \mathrm{yr}$ (Loarie et al. 2009), so the spreading speed of Scotch Broom is well below the velocity of climate change. Thus, Scotch Broom falls under the conditions of Theorem II.1. This means that Scotch Broom will experience a northern range contraction in Australia. This result was also predicted by Potter et al. (2008).

Compared with Scotch Broom, the light brown apple moth (LBAM, Epiphyas postvittana) is another example of a species facing range encroachment. Loarie et al. (2009) showed that the estimated maximum spreading speed of LBAM is $6 \mathrm{~km} / \mathrm{yr}$, which is well above the velocity of climate change. Thus, LBAM falls under the conditions of Theorem II.2. This means that LBAM will continue to spread with a 
northward spreading speed which is determined by the inherent LBAM rate of spread and the velocity of climate change. Since extreme summer temperatures may decrease the spreading speed of LBAM, especially in the southwestern USA (Lozier and Mills 2011), the southward spreading speed of LBAM is lower than the northward spreading speed.

For the second scenario, we demonstrated that the spread of a species into less favorable habitat requires that the species expand at a rate faster than the climate expansion rate. The spreading speed of species within their less favorable habitat is determined by how well the species can survive in the most extreme poor-quality environment. One good example for species facing range expansion and spread into less favorable habitat is the Hemlock Woolly Adelgid (HWA). HWA can destroy vast forests of hemlock and affiliated communities within their habitat, while extreme cold temperatures could prevent HWA's spread. Many HWA die off in the winter, having laid eggs that will hatch in the spring and also the spreading speed of HWA at the northern range boundary is relatively low. These reduce their population growth rate during the next spring and also restrict the northward spread of HWA. However, global warming provides opportunities for HWA to spread into currently untouched forests. Because the spreading speed of HWA is faster than the velocity of climate change (Evans and Gregoire 2007; Loarie et al. 2009), from Theorem II.4 and II.5, we predict that the northward spreading speed of HWA will be determined by the inherent LBAM rate of spread in less favorable habitat and the velocity of climate change. Similar results have been obtained by Paradis et al. (2008).

Compared with HWA, The Mediterranean Gecko (Hemidactylus turcicus) is another example of a species facing range expansion (Meshaka et al. 2006). Locey and Stone (2006) showed that the estimated spreading speed of this species is $20 \mathrm{~m} / \mathrm{yr}$, which is much slower than the velocity of climate change(Loarie et al. 2009). From Theorem II.3, we predicted that Gecko will spread by its ability to colonize a new location. 


\section{Future Directions}

Results in this dissertation can be extended in several different directions:

1. Periodicity in space or in time. Periodic phenomena are very common in biology. They can appear in a heterogeneous environment which is composed of two kinds of patches with different diffusivity and intrinsic growth rates, that vary periodically (Shigesada et al. 1986). Periodicity can also happen in time, for example, seasonality. Seasonal reproduction in mammals has been affected by climate change and many species may face extinction if they cannot develop new strategies for seasonal changes (Bronson 2009; Bradshaw and Holzapfel 2006). We can discuss the persistence and spread of species by considering periodicity in space or in time, for instance by letting $r(x, t)$ to be periodic in $x$ or $t$.

2. Density-dependent diffusion rate. Aggregation is an important social behavior in many species. Actually, it is hard to find animals that do not aggregate in nature. Individuals tend to join together at low densities and repel each other at high density. If the motion of individuals is influenced by their neighbors, their motion is said to be density dependent; otherwise, their motion is density independent. For density dependent aggregation, individuals attract each other due to social interactions, for example, mating, settlement and so on, which can help the population defend against predators (Fedotov et al. 2008). The most common theoretical model to explain the species' aggregation behavior is known as the density-dependent Fisher equation. This equation requires only one partial differential equation for the individuals' motion but is nonlinear due to density dependent diffusivity $D(u)$ (Gurney and Nisbet 1975; Shigesada et al. 1979; Petrovskii and Li 2003; Almeida et al. 2006; Balasuriya and Gottwald 2010; Kenkre and Kumar 2008).

3. Allee effect. The Allee effect is the positive correlation between population size or density and the mean population growth rate per capita of a species(Stephens 
1999). The Allee effect means that when the population density is low, the growth rate of the population is not optimal. This phenomenon is usually observed in species that require the assistance of another individual; for example species that hunt in packs would not be able to survive in smaller groups (Berec 2007). The steady state distribution of reaction-diffusion equations with Allee effect growth has been studied (Ali et al. 2009) and it was also found that the Allee effect can speed up or slow down population growth rate and invasions(Allee 1932; Shaw and Kokko 2015). This could be incorporated by a reaction term with the Allee effect.

\section{Further scenarios for two species competing along shifting habitats.} In chapter III, we provided a very basic analysis and discussed the simplest questions for a competition model along shifting habitat edges. Further analytic explorations of the various scenarios regarding which direction habitats shift, how resources are distributed, the competition coefficients of both species, etc., could be elaborated as well. We provided examples of these at the end of Chapter III.

5. Stage-structured models with phonological variations. Another possible direction is to consider the age structure for individuals in the model, i.e. dividing populations of species into adults and juveniles. In this model, only adult members of the population can reproduce. There might be no direct interaction between adults and juveniles or they have to compete for common resources. Some adults (black iguanas) will even eat juveniles during times of scarce food sources. Their life-cycle takes place in a year and can be affected by seasonality. For example, some species will be dormant in the fall and winter, and they will grow and spread in the spring and summer(Meyer and Li 2013).

Future research based on the findings of this dissertation, as has been shown, could take multiple directions and thus the method represented in this work holds fruitful possibilities for further exploration. 


\section{REFERENCES}

[1] Ali, J., Shivaji, R., Wampler, K. (2009). Population models with diffusion, strong Allee effect and constant yield harvesting. Journal of Mathemaical Analysis and Applications 352, 907-913.

[2] Allee, W.C., \& Bowen E. (1932). Studies in animal aggregations: mass protection against colloidal silver among goldfishes. Journal of Experimental Zoology 61(2), 185-207.

[3] Almeida, R.C., Delphim, S.A., \& da Costa, M.I.S. (2006). A numerical model to solve single-species invasion problems with Allee effects. Ecological Modeling 192, 601-617.

[4] Al-Khaled, K. (2001). Numerical study of Fisher's reactiondiffusion equation by the Sinc collocation method. Journal of Computational and Applied Mathematics $137(2), 245-255$.

[5] Andow, D. A., Kareiva, P. M., Levin, S.A., \& Okubo, A. (1990). Spread of invading organisms. Landscape Ecology 4, 177-188.

[6] Araujo, M.B., \& Pearson, R.G. (2005). Equilibrium of species distributions with climate. Ecogeography 28, 693-695.

[7] Aronson, D.G., \& Weinberger, H.F. (1975). Nonlinear diffusion in population genetics, combustion, and nerve impulse propagation in Partial Differential Equations and Related Topics. Lecture Notes in Mathematics. Springer-Verlag, Berlin, 446, 5-49.

[8] Aronson, D.G., \& Weinberger, H.F. (1977). The asymptotic speed of propagation of a simple epidemic. Nonlinear diffusion (NSF-CBMS Regional Conf. Nonlinear Diffusion Equations, Univ. Houston, Houston, Tex., 1976), 1-23. Research Notes in Mathematics, 14. Pitman, London. 
[9] Aronson, D.G., \& Weinberger, H.F. (1978). Multidimensional nonlinear diffusion arising in population genetics. Advances in Mathematics 30, 33-76.

[10] Aronson, D.G., \& Huang, Y.S. (1994). Limit and uniqueness of discrete rotating waves in large arrays of Josephson junctions. Nonlinearity 7, 777-804.

[11] Baker, R., \& Dunn, P. (1990). New directions in biological control. Alan Liss, New York, 1990.

[12] Balasuriya, S., \& Gottwald, G.A. (2010). Wavespeed in reaction-diffusion systems, with applications to chemotaxis and population pressure. Journal of Mathematical Biology 61, 377-399.

[13] Bradley, B.A., Wilcove, D.S., Oppenheimer, M. (2010) Climate change increases risk of plant invasion in the eastern United States. Biological Invasions 12, 18991872 .

[14] Bradshaw W. E., Holzapfel C. M. (2006). Evolutionary response to rapid climate change. Science 312, 1477-1478.

[15] Bell, J. (1981). Some threshold results for models of myelinated nerves. Mathematical Biosciences 54, 181-190.

[16] Berec L., Angulo E., Courchamp F. (2007). Multiple Allee effects and population management. Trends in Ecology and Evolution 22(4), 185-191.

[17] Berestycki, H., Diekmann, O, Nagelkerke, C.J., \& Zegeling, P.A. (2009). Can a species keep pace with a shifting climate? Bulletin of Mathematical Biology 71, 399-429.

[18] Bronson F.H. (2009). Climate change and seasonal reproduction in mammals. Philosophical Transactions of the Royal Society B 364, 3331-3340.

[19] Cahn, J. W. (1960). Theory of crystal growth and interface motion in crystalline materials. Acta Metallurgica 8, 554-562. 
[20] Cahn, J.W., Mallet-Paret, J., \& Van Vleck, E.S. (1998). Traveling wave solutions for systems of ODEs on a two-dimensional spatial lattice. SIAM Journal on Applied Mathematics 59, 455-493.

[21] Cantrell, R.S., \& Cosner, C. (2003). Spatial Ecology via Reaction-Diffusion Equations. Department of Mathematics, University of Miami, U.S.A., (2003).

[22] Carr, J., \& Chmaj, A. (2004). Uniqueness of traveling waves for nonlocal monostable equations. Proceeding of the American Mathematical Society 132, 24332439.

[23] Chen, X., \& Guo, J.S. (2002). Existence and asymptotic stability of traveling waves of discrete quasilinear monostable equations. Journal of Differential Equations 184, 549-569.

[24] Chi, H., Bell, J., \& Hassard, B. (1986). Numerical solution of a nonlinear advance-delay-differential equation from nerve con-duction theory. Journal of Mathematical Biology 24, 583-601.

[25] Chow, S.N., Mallet-Paret, J., \& Shen, W. (1998) Traveling waves in lattice dynamical systems, Journal of Differential Equations 149, 248-291.

[26] Cook, H.E., de Fontaine, D., \& Hilliard, J.E. (1969). A model for diffusion on cubic lattices and its application to the early stages of ordering. Acta Meterialia 17, 765-773.

[27] Van Dover, C.L. (2014). Impacts of anthropogenic disturbances at deep-sea hydrothermal vent ecosystems: A review. Marine Environmental Research 102, $59-72$.

[28] Downey, P.O., \& Smith, J.M.B. (2000). Demography of the invasive shrub scotch broom (Cytisus scoparius) at Barrington Tops, New South Wales: insights for management. Austral Ecology 25, 477-485. 
[29] Evans, A.M., \& Gregoire, T.G. (2007). A geographically variable model of hemlock woolly adelgid spread. Biological Invasions 9, 369-382.

[30] Fagan, W.F., Cantrell, R.S., \& Cosner, C. (1999). How habitat edges change species interactions. American Naturalist 153, 165-182.

[31] Fagan, W.F., Cantrell, R.S., Cosner, C., \& Ramakrishnan, S. (2009). Interspecific variation in critical patch size and gap crossing ability as determinants of geographic range size distributions. The American Naturalist 173, 363-375.

[32] Fang, J., Wei, J. \& Zhao, X.-Q. (2010). Spreading speed and traveling waves for non-monotone time-delayed lattice equations. Proceeding of the royal Society of London A: Mathematical, Physical and Engineering Sciences 466, 1919-1934.

[33] Fedotov, S., Moss,D., \& Campos,D. (2008). Stochastic model for population migration and the growth of human settlements during the Neolithic transition. Physical Review E 78, 026-107.

[34] Fick, A. (1855). "On liquid diffusion". Poggendorffs Annalen 94, 59. reprinted in Journal of Membrane Science 100, 33-38. 1995.

[35] Fisher, R.A. (1937). The wave of advance of advantageous genes. Annals of Human Genetics 7, 353-369.

[36] Gonzalez, P., Neilson, R.P., Lenihan, J.M., \& Drapek, R.J. (2010). Global patterns in the vulnerability of ecosystems to vegetation shifts due to climate change. Global Ecology Biogeography 19, 755-768.

[37] Greenstein, B.J., \& Pandolfi, J.M. (2008). Escaping the heat: range shifts of reef coral taxa in coastal Western Australia. Global Change Biology 14, 513-528.

[38] Grindrod, P. (1996). The Theory and Applications of Reaction-Diffusion Equations, Oxford: Clarendon Press.

[39] Gurney, W.S.C., \& Nisbet,R.M. (1975). The regulation of inhomogeneous populations, Journal of Theoretical Biology 52, 441-457. 
[40] Hodkinson, I.D. (2005). Terrestrial insects along elevation gradients: species and community responses to altitude. Biological Reviews 80, 489-513.

[41] Hu, C., \& Li, B. (2015). Spatial dynamics for lattice differential equations with a shifting habitat. Journal of Differential Equation 259, 1967-1989.

[42] Huang, J., \& Shen, W. (2009) Speeds of spread and propagation of KPP models in time almost and space periodic media. SIAM Journal on Applied Dynamical Systems 8, 790-821.

[43] Iooss, G. (2000). Traveling waves in the Fermi-Pasta-Ulam lattice, Nonlinearity 13, 849-866.

[44] Kan-on, Y. (1996). Existence of standing waves for competition-diffusion equations. Japan Journal of Industrial and Applied Mathematics 13, 117-133.

[45] Keener, J.P. (1987). Propagation and its failure in coupled systems of discrete excitable cells. SIAM Journal on Applied Mathematics 47, 556-572.

[46] Keleher, C.J., \& Rahel, F.J. (1996). Thermal limits to salmonid distributions in the Rocky Mountain region and potential habitat loss due to global warming: a geographic information system (GIS) approach. Transactions of the American Fisheries Society 125, 1-13

[47] Kenkre, V.M., \& Kumar,N. (2008). Nonlinearity in bacterial population dynamics: proposal for experiments for the observation of abrupt transitions in patches. Proceeding of the National Academy of Sciences of the United States of America 105, 1875218757.

[48] Kolmogorov, A., Petrovskii, I., \& Piscounov, N. (1937). Etude de lequation de la diffusion avec croissance de la quantite de matiere et son application a un probleme biologique. Moscow University Mathematics Bulletin 1(126).

[49] Kolmogorov, A., Petrovskii, I., \& Piscounov, N. (1991). A study of the diffusion equation with increase in the amount of substance, and its application to a biolog- 
ical problem. In V. M. Tikhomirov, editor, Selected Works of A. N. Kolmogorov I, Kluwer, 248-270.

[50] Laplante, J.P., \& Erneux, T. (1992). Propagation failure in arrays of coupled bistable chemical reactors. The Journal of Physical Chemistry 96, 4931-4934.

[51] Lewis, M.A., Schmitz, G., Kareiva, P., \& Trevors, J.T. (1996). Models to examine containment and spread of genetically engineered microbes. Molecular Ecology $5,165-175$.

[52] Lewis, M.A., Li, B., \& Weinberger, H.F. (2002). Spreading speeds and the linear conjecture for two-species competition models. Journal of Mathematical Biology 45, 219-233.

[53] Lewis, M.A., \& Li, B. (2012). Spreading speed, traveling waves, and minimal domain size in impulsive reaction-diffusion models. Bulletin of Mathematical Biology 74, 2383-2402.

[54] Li, B, Weinberger, H.F., \& Lewis, M.A. (2005). Spreading speeds as slowest wave speeds for cooperative systems. Mathematical Biosciences 196(1), 82-98.

[55] Li B, Bewick S, Shang J, \& Fagan W.F. (2014). Persistence and spread of a species with a shifting habitat edge. SIAM Journal of Applied Mathematics 74, 1397-1417. Li B, Bewick S, Shang J, Fagan W.F. (2015). Erratum to: Persistence and spread of a species with a shifting habitat edge. SIAM Journal of Applied Mathematics 75, 2379-2380.

[56] Li B, Bewick S, Barnard R.M., \& Fagan W.F. (2016). Persistence and spreading speeds of integro-difference equations with an expanding or contracting habitat. Bulletin of Mathematical Biology 78(7), 1337-1379.

[57] Liang, X., \& Zhao, X.-Q. (2007). Asymptotic speeds of spread and traveling waves for monotone semifolows with applications, Communications on Pure and Applied Mathematics 60, 1-40. 
[58] Loarie, S.R., Duffy, P.B., Hamilton, H., Asner, G.P., Field, C.B., \& Ackerly, D.D. (2009). The velocity of climate change, Nature 462, 1052-1055.

[59] Locey, K.J., \& Stone, P.A. (2006). Factors affecting range expansion in the introduced Mediterranean gecko, Hemidactylus turcicus, Journal of Herpetology 40, 526-530.

[60] Lozier, J.D., \& Mills, N.J. (2011). Predicting the potential invasive range of light brown apple moth (Epiphyas postvittana) using biologically informed and correlative species distribution models. Biological Invasions 13, 2409-2421.

[61] Lui R. (1989). Biological growth and spread modeled by systems of recursions. I Mathematical theory. Mathematical Biosciences 93, 269-295.

[62] Lui R. (1989). Biological growth and spread modeled by systems of recursions. II Mathematical theory. Mathematical Biosciences 93, 297-312.

[63] Lynch, H.J., Naveen, R., Trathan, P.N., and Fagan, W.F. (2012). Environmental change and the shifting balance among penguins on the Antarctic Peninsula. Ecology 93, 1367-1377.

[64] Ma, S., \& Zou X. (2005). Propagation and its failure in a lattice delayed differential equation with global interaction. Journal of Differential Equations 212, 129-190.

[65] Mallet-Paret, J. (1999). The global structure of traveling waves inspatially discrete dynamical systems. Journal of Dynamics and Differential Equations 11, $49-128$.

[66] Meshaka, W.E. Jr, Marshall, S.D., Boundy, J., \& Williams, A.A. (2006). Status and geographic expansion of the Mediterranean Gecko, Hemidactylus turcicus, in Louisiana: implications for the southeastern United States. Herpetological Conservation and Biology 1, 4550. 
[67] Meyer, K., \& Li, B. (2013). A Spatial Model of Plants with an Age-Structured Seed Bank and Juvenile Stage, SIAM Journal on Applied Mathematics 73, 16761702.

[68] Mooney, H.A., \& Hobbs, R.J. (2000). Invasive species in a changing world, Island Press, London.

[69] Morita, Y., \& Tachibana, K. (2009). An Entire Solution to the LotkaVolterra Competition-Diffusion Equations. SIAM Journal on Mathmatical Analysis 40(6), $2217-2240$.

[70] Morrison, L.M., Korzukhin, M.D., \& Porter, S.D. (2005). Predicted range expansion of the invasive fire ant, Solenopsis invicta, in the eastern United States based on the VEMAP global warming scenario. Diversity Distributions 11, 199-204.

[71] Murray J. D. (2002). Mathematical biology I: an introduction. Berlin: Springer.

[72] Murray J. D. (2002). Mathematical biology II: spatial models and biomedical applications. Berlin: Springer.

[73] Murray, J.D. (2012). Mathematical Biology, 3rd edition, vols. 1 and 2, Berlin and New York: Springer.

[74] Neubert, M.G., \& Parker, I.M. (2004). Projecting rates of spread for invasive species. Risk Analysis 24, 817-831.

[75] Ni, J. (2000). A simulation of biomes on the Tibetan Plateau and their responses to global climate change. Mountain Research and Development 20, 80-89.

[76] Orwig, D.A., Foster, D.R., \& Mausel, D.L. (2002). Landscape patterns of hemlock decline in New England due to the introduced hemlock woolly adelgid. Journal of Biogeography 29, 1475-1487.

[77] Pao, C.V. (1992). Nonlinear Parabolic and Elliptic Equations, Plenum Press, New York. 
[78] Pao, C.V. (1998). Parabolic Systems in Unbounded Domains I. Existence and Dynamics. Journal of Mathematical Analysis and Applications 217(1), 129-160.

[79] Paradis, A., Elkinton, J., Hayhoe, K., \& Buonaccorsi, J. (2008). Role of winter temperatures and climate change on the survival and future range expansion of the hemlock wooly adelgid (Adelges tsugae) in eastern North America. Mitigation Adaptation Strategies Global Change 13, 541-554.

[80] Parmesan, C. (2006). Ecological and evolutionary responses to recent climate change. Annual Review of ecology, Evolution, and Systematics 37, 637-669.

[81] Parr, C.L., Gray, E.F. \& Bond, W.J. (2012). Cascading biodiversity and functional consequences of a global change-induced biome switch. Diversity Distributions 18, 493-503.

[82] Petrovskii, S.V., \& Li, B. (2003). An exactly solvable model of population dynamics with density-dependent migrations and the Allee effect. Mathematical Biosciences 186, 79-91.

[83] Polovina, J.J., Dunne, J.P., Woodworth, P.A., \& Howell, E.A. (2011). Projected expansion of the subtropical biome and contraction of the temperate and equatorial upwelling biomes in the North Pacific under global warming. ICES Journal of Marine Science 68, 986-995.

[84] Potapov, A.B., \& Lewis, M.A. (2004). Climate and competition: The effect of moving range boundaries on habitat invisibility. Bulletin of Mathematical Biology 66, 975-1008.

[85] Potter, K.J.B., Kriticos, D.J., \& Leriche, A. (2008). Climate change impacts on Scotch broom in Australia. In: Proceedings of the 16th Australian weeds conference, Cairns Convention Centre, North Queensland, Australia, 1822 May, Queensland Weed Society

[86] Quintero, I. \& Wiens, J.J. (2013). Rates of projected climate change dramatically 
exceed past rates of climatic niche evolution among vertebrate species. Ecology Letters 16(8), 1095-1103.

[87] Rahel, F.J., \& Olden, J.D. (2008). Assessing the effects of climate change on aquatic invasive species. Conservation Biology 22, 521-533.

[88] Rejmanek, M., \& Richardson, D.M. (1996). What Attributes Make Some Plant Species More Invasive? Ecology 77, 1655-1661.

[89] Scheel, A., \& van Vleck, E. (2009). Lattice Differential Equations Embedded Into Reaction Diffusion Systems. Proceedings of The Royal Society of Edinburgh, Section: A 139A, 193-207.

[90] Scheffer, M., Hirota, M., Holmgren, M., Van Nes, E.H., \& Chapin, F.S. (2012). Thresholds for boreal biome transitions, PNAS, Proceedings of the National Academy of Science USA 109, 21384-21389.

[91] Scheiter, S. \& Higgins, S.I. (2009). Impacts of climate change on the vegetation of Africa: An adaptive dynamic vegetation modelling approach. Global Change Biology 15, 2224-2246.

[92] Scott, A. (2003). Nonlinear Science: Emergence and Dynamics of Coherent Structure, 2nd edition. Oxford and New York: Oxford University Press.

[93] Shaw, A.K., \& Kokko, H. (2015). Dispersal Evolution in the Presence of Allee Effects Can Speed Up or Slow Down Invasions. The American Naturalist 185(5), 631-639.

[94] Shigesada, N., Kawasaki, K., \& Teramoto, E. (1979). Spatial segregation of interacting species. Journal of Theoretical Biology 79, 83-99.

[95] Shigesada, N., Kawasaki, K., \& Teramoto, E. (1986). Traveling periodic waves in heterogeneous environments. Theoretical Population Biology 30, 143-160.

[96] Shigesada, N., \& Kawasaki, K. (1997). Biological invasions: theory and practice. Oxford: Oxford University Press. 
[97] Smale, D.A., \& Wernberg, T. (2013). Extreme climatic event drives range contraction of a habitatforming species. Proceedings of the Royal Society of London B: Biological Sciences 280.

[98] Stephens, P.A., Sutherland, W.J., \& Freckleton, R.P. (1999). What is the Allee effect? Oikos, 185-190.

[99] Svenning, J.C., \& Skov, F. (2007). Ice age legacies in the geographical distribution of tree species richness in Europe. Global Ecology Biogeography 16, 234-245.

[100] Svenning, J.C., \& Skov, F. (2007). Could the tree diversity pattern in Europe be generated by postglacial dispersal limitation? Ecology Letters 10, 453-460.

[101] Teif, V.B., \& Rippe K. (2010). Statistical-mechanical lattice models for proteinDNA binding in chromatin. Journal of Physics: Condensed Matter 22(41), 414105.

[102] Tng, D.Y.P., Murphy, B.P., Weber, E., Sanders, G., Williamson, G.J., Kemp, J., \& Bowman, D.M.J.S. (2012). Humid tropical rain forest has expanded into eucalypt forest and savanna over the last 50 years, Ecology Evolution 2, 34-45.

[103] Verhulst, P.F. (1845). Recherches mathmatiques sur la loi d'accroissement de la population. Nouv. mm. de l'Academie Royale des Sci. et Belles-Lettres de Bruxelles 18, 1-41.

[104] Verhulst, P.F. (1847). Deuxime mmoire sur la loi d'accroissement de la population. Mm. de l'Academie Royale des Sci., des Lettres et des Beaux-Arts de Belgique 20, 1-32.

[105] Wang, X.F. (1993). On the Cauchy Problem for Reaction-Diffusion Equations. Transactions of the American Mathematical Society 337(2), 549-590.

[106] Weinberger, H.F. (1982). Long-time behavior of a class of biological models. SIAM Journal on Mathematical Analysis (SIMA) 13, 353-396. 
[107] Weinberger, H.F., Lewis, M.A., \& Li, B. (2002). Analysis of the linear conjecture for spread in cooperative models. Journal of Mathematical Biology 45, 183-218.

[108] Weng, P.X., Huang, H.X., \& Wu, J.H. (2003). Asymptotic speed of propagation of wave fronts in a lattice delay differential equation with global interaction. IMA Journal of Applied Mathematics 68, 409-439.

[109] With K.A. (2002). The landscape ecology of invasive spread. Conservation Biology, 16, 1192-1203.

[110] Winslow, R.L., Kimball, A.L., \& Varghese, A. (1993). Simulating cardiac sinus and atrial network dynamics on the Connection Machine. Physica D 64, 281-298.

[111] Wu, J., Zou, X. (1997). Asymptotic and periodic boundary value problems of mixed FDEs and wave solutions of lattice differential equations. Journal Differential Equations 135, 315-357.

[112] Zhang, B.T., Yang, J, \& Chi, S.W. (2003). Self-Organizing Latent Lattice Models for Temporal Gene Expression Profiling. Machine Learning 52(1), 67-89.

[113] Zhang, L., \& Guo, S. (2014). Existence and multiplicity of wave trains in 2D lattices. Journal of Differential Equations 257, 759-783.

[114] Zhou, Y., \& Kot, M.(2011). Discrete-time growth-dispersal models with shifting species ranges. Theoretical Ecology 4, 13-25.

[115] Zinner, B. (1992). Existence of travling wave front solutions for the discrete Nagumo equation. Journal of Differential equations 96, 1-27.

[116] Zinner, B., Harris, G., \& Hudson, W. (1992). Traveling wave fronts for the discrete Fisher's equation. Journal of Differential Equations 105, 46-62. 


\title{
CURRICULUM VITAE
}

\author{
Jin SHANG
}

\section{Education}

University of Louisville, Louisville, KY

Ph.D., Applied and Industrial Mathematics,

Fall 2016

M.A., Applied and Industrial Mathematics,

Sichuan University, Sichuan, China

M.A., College of Mathematics,

June 2010

Hebei Normal University, Hebei, China

B.S., College of Mathematics and Information Science,

June 2007

\section{Professional Experience}

Business Data Analyst

$2015-2016$

Fruit of the Loom, Bowling Green, KY

Graduate Teaching Assistant, University of Louisville, Louisville, KY

Math 111: College Algebra Aug. 2010 - Sep. 2015

Math 109: Elementary Statistics

Math 180: Elements of Calculus

Graduate Research Assistant

University of Louisville, Louisville, KY

Research Experience

University of Louisville, Louisville, $\mathrm{KY}$

Private Tutor and University of Louisville Math Lab Tutor

Louisville, KY

Aug. 2010 - Sep. 2015 


\section{Talks \& Presentations}

Period-doubling Bifurcation and Period-undoubling

Bifurcation in a Discrete-time Model

Oct. 2013

AMS Southeastern Sectional Meeting, University of Louisville, Louisville, KY

\section{Professional Memberships}

American Mathematical Society

Since 2010

Tri-State Actuarial Club

Since 2012

Math Club

Since 2012

\section{RESEARCH ACHIEVEMENT}

Bifurcations in a discrete time model composed of Beverton-Holt function and Ricker function J. Shang, B. Li, and M. Barnard, Mathematical Biosciences (2015)

Persistence and spread of a species with a shifting habitat edge B. Li, S. Bewick, J. Shang, and W. F. Fagan, SIAM Journal on Applied Mathematics, 74 (2014), 1397-1417.

Stability and traveling wave solutions for a time-delayed populations system with stage structure Liang Zhang, Bingtuan Li, Jin Shang, Nonlinear Analysis: Real world and applications, 13 (2012) 1429-1440.

Analytic vector field on compact smooth toric varieties Jin Shang, Xinhong Chen, Journal of Sichuan University(Natural Science Edition), 49(3), 2012.

The orbifold construction of weighting toric varieties Xinhong Chen, Jin Shang, Journal of Sichuan University(Natural Science Edition), 48(6), 2011. 